\title{
Cape Verde: 2010 Article IV Consultation and Request for a 15-Month Policy Support Instrument-Staff Report; Public Information Notice and Press Release on the Executive Board Discussion; and Statement by the Executive Director for Cape Verde.
}

Under Article IV of the IMF's Articles of Agreement, the IMF holds bilateral discussions with members, usually every year. In the context of a combined discussion of the 2010 Article IV consultation with Cape Verde and Request for a 15-Month Policy Support Instrument, the following documents have been released and are included in this package:

- $\quad$ The staff report for the combined 2010 Article IV consultation and Request for a 15-Month Policy Support Instrument, prepared by a staff team of the IMF, following discussions that ended on September 28, 2010, with the officials of Cape Verde on economic developments and policies. Based on information available at the time of these discussions, the staff report was completed on November 9, 2010. The views expressed in the staff report are those of the staff team and do not necessarily reflect the views of the Executive Board of the IMF.

- $\quad$ A Public Information Notice (PIN) and Press Release, summarizing the views of the Executive Board as expressed during its November 22, 2010, discussion of the staff report on issues related to the Article IV consultation and the IMF arrangement, respectively.

- $\quad$ A statement by the Executive Director for Cape Verde.

The documents listed below have been or will be separately released.

Letter of Intent sent to the IMF by the authorities of Cape Verde*

Memorandum of Economic and Financial Policies by the authorities of Cape

Verde*

Technical Memorandum of Understanding*

*Also included in Staff Report

The policy of publication of staff reports and other documents allows for the deletion of market-sensitive information.

\author{
Copies of this report are available to the public from \\ International Monetary Fund • Publication Services \\ $70019^{\text {th }}$ Street, N.W. $\bullet$ Washington, D.C. 20431 \\ Telephone: (202) 623-7430 • Telefax: (202) 623-7201 \\ E-mail: publications@imf.org Internet: http://www.imf.org
}

\section{International Monetary Fund Washington, D.C.}




\title{
INTERNATIONAL MONETARY FUND
}

\section{CAPE VERDE}

\section{Staff Report for the 2010 Article IV Consultation and Request for a 15-Month Policy Support Instrument}

\author{
Prepared by the African Department \\ (In consultation with other departments) \\ Approved by Sharmini Coorey and James Roaf
}

November 9, 2010

\section{Executive Summary}

Focus: Following the expiration of their previous PSI, the authorities have requested a 15-month PSI supported program, which will allow the new government to assess the form of its future engagement with the Fund after elections in 2011. The authorities remain firmly committed to macroeconomic stability and view the program as a useful macroeconomic anchor, which would focus on keeping net domestic debt low and building international reserves.

Assessment: Cape Verde weathered the global economic crisis relatively well because of the fiscal and reserves buffers built before the crisis and due to counter-cyclical macroeconomic policies. Their temporary public investment boom, which is mainly foreign-financed, makes use of the concessional resources that are available over the next few years as the country transitions to a middle-income economy. Staff supports the request for a new PSI supported program.

\section{Policy discussions:}

- Fiscal policy. The key policy challenge will be to execute the public investment program efficiently, and exit from the fiscal stimulus over the next few years as planned to maintain debt sustainability.

- Monetary policy. Despite a large spread with the Euribor, the central bank remains cautious of monetary easing as it seeks to stabilize inflows of emigrant deposits and to reinforce the buildup of foreign exchange reserves.

- Structural reforms. Reforms will focus on improving debt management capability; strengthening monetary operations; and safeguarding financial sector stability.

Exchange rate regime: Conventional peg to the euro.

\footnotetext{
${ }^{1}$ Discussions took place during September 15-28, 2010. The team comprised Valerie Cerra (head), Abdullah AlHassan, Fabiano Bastos, and Jean-Baptiste Le Hen. The mission met with Cristina Duarte, Minister of Finance; Carlos Burgo, Governor of the Bank of Cape Verde; other government officials; and representatives of the private sector. The mission conducted outreach activities with the press and other members of the local community.
} 




II. Recent Developments and Outlook ...............................................................

A. Recovering from the Global Slowdown.....................................................



III. Policy Discussions: Rebuilding Buffers and Developing Markets ............................. 7

A. Fiscal Policy: Improving Infrastructure While Maintaining Fiscal Sustainability ...7

B. Monetary Policy: Building and Stabilizing Reserves .........................................10

C. Safeguarding Financial Stability and Developing Markets.................................11

D. Reducing Fiscal Risk in State-Owned Enterprises ...........................................13

E. Social Policies to Protect the Vulnerable ...........................................................13

F. Sustaining Medium-term Growth ............................................................ 14



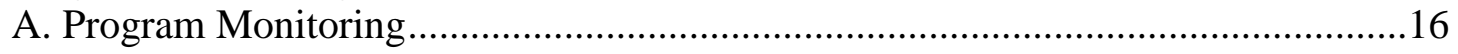

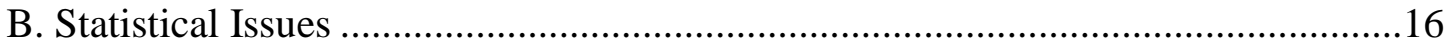





Tables

1. Selected Indicators. .........................................................................................22

2. Fiscal Operations of the Central Government. ............................................................23

3. Fiscal Operations of the Central Government (Percent).........................................24

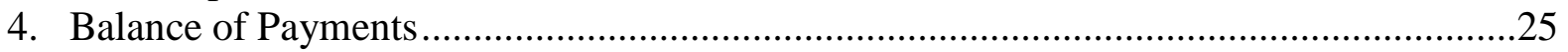

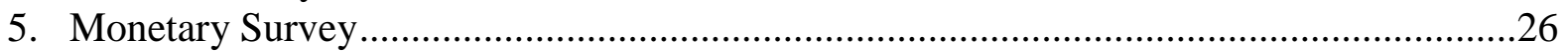

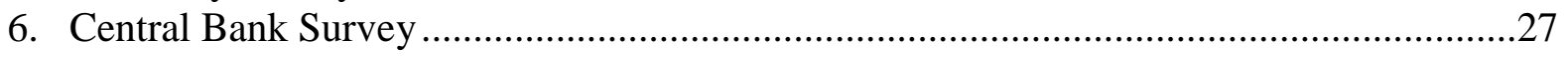

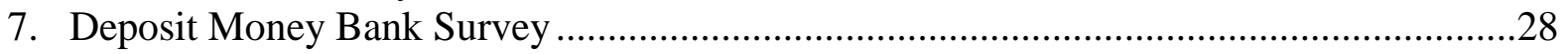

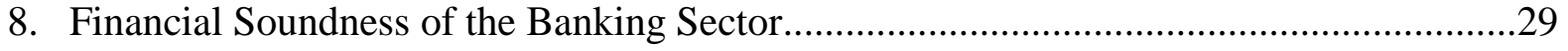

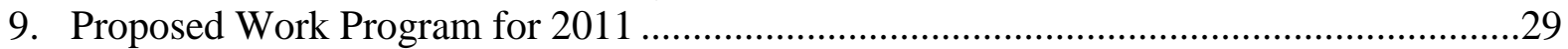

10. Quantitative Assessment Criteria and Indicative Targets for 2010-11



Figures

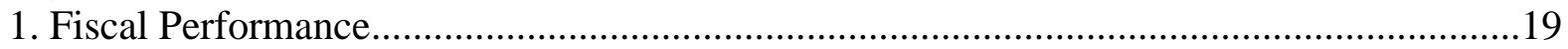

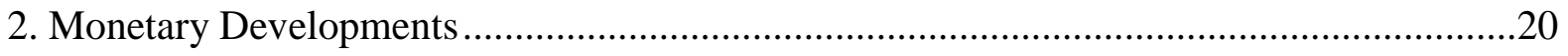

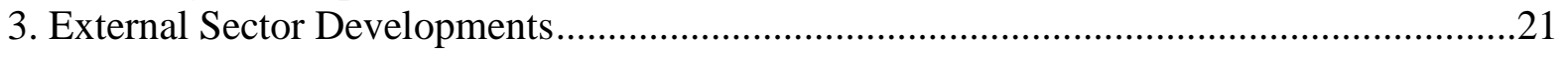

Boxes

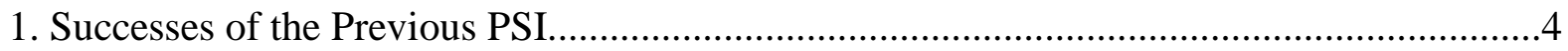

2. Assessment of the Exchange Rate ...........................................................................6

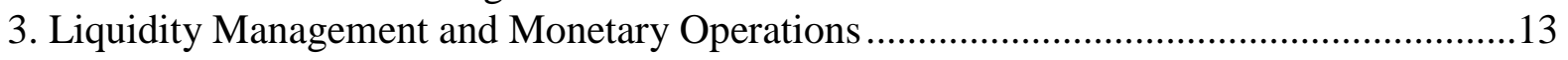

4. Cape Verde's Social Policies ............................................................................... 15 
Appendixes



Attachment I. Memorandum of Economic and Financial Policies ..................................32

Attachment II. Technical Memorandum of Understanding .........................................39








\section{INTRODUCTION}

1. Performance under the 2006-10 Policy Support Instrument (PSI) was strong.

International reserves grew to over four months of imports and net domestic debt declined to less than 20 percent of GDP. A prudent fiscal stance during the first years of the program allowed effective counter-cyclical policies in 2008-09 when the global crisis hit.

\section{Box 1. Successes of the Previous PSI}

In 2006, after completion of a three-year PRGF arrangement, Cape Verde obtained a three-year PSI supported program, which was extended by one year in 2009. Under this previous PSI, covering the period 2006-10, policy measures enhanced macroeconomic performance in several areas, including: (i) reducing public debt, (ii) building international reserves, (iii) improving tax policies and public financial management, and (iv) strengthening regulatory capacity in the financial sector.

A key reason for the success of the previous PSI was the authorities' ability to leverage its own reform agenda taking advantage of the technical discussions, structural benchmarks, and performance criteria associated with the program. PSI-supported policies helped to consolidate macroeconomic stability, which is at the center of the government's poverty reduction strategy.

2. The authorities are requesting a new 15-month PSI. Legislative and presidential elections are scheduled for early 2011, and the authorities have requested a 15-month PSI supported program, which will allow the new government to assess the form of its future engagement with the Fund after elections in 2011. With only a notional budget for 2011, they consider the macroeconomic framework and conditionality of the PSI supported program as useful policy anchors. ${ }^{2}$ The new program would continue to focus on macroeconomic stability, and build on the successes of the previous program.

\section{RECENT DEVELOPMENTS AND OUTLOOK}

\section{A. Recovering from the Global Slowdown}

3. Economic growth in $\mathbf{2 0 1 0}$ shows signs of a solid recovery. In recent months, both quantitative and qualitative economic indicators point to clear signs of recovery in economic activity. Leading economic indicators, such as the economic climate indicator and confidence indicators by economic sectors, reveal a favorable performance in the first half of 2010. Lower prices in the tourism industry have sustained growth in hotel nights and visiting guests. In the transportation sector, passenger arrivals and cargo activities have been robust. High growth in

\footnotetext{
2 The national assembly will not vote on a budget for 2011 until the new government is formed, which is unlikely until mid-2011. In the meantime, budget execution will follow the medium term fiscal framework (MTFF) that was included in the budget write up and attached to the submission of the 2010 budget to the national assembly.
} 
imports of consumer, intermediate, and capital goods herald strong domestic demand. Notably, rapid growth of cement imports portends a rise in construction activity and investment.



\section{Inflation edged up in recent months, but remains low and the real value of the} escudo remains competitive. Inflation has increased to 3.0 percent in the 12 months to August 2010, mainly because of higher prices in service activities (i.e. restaurants and transportation sectors) and food. The implementation of the public investment program (PIP) should continue to have little effect on domestic inflation due to the high import content of investment in terms of inputs of goods, labor, and other services. The nominal effective exchange rate depreciated slightly in recent months due to the peg to the euro, which has depreciated relative to the dollar. The real effective exchange rate is broadly in line with fundamentals (Box 2).
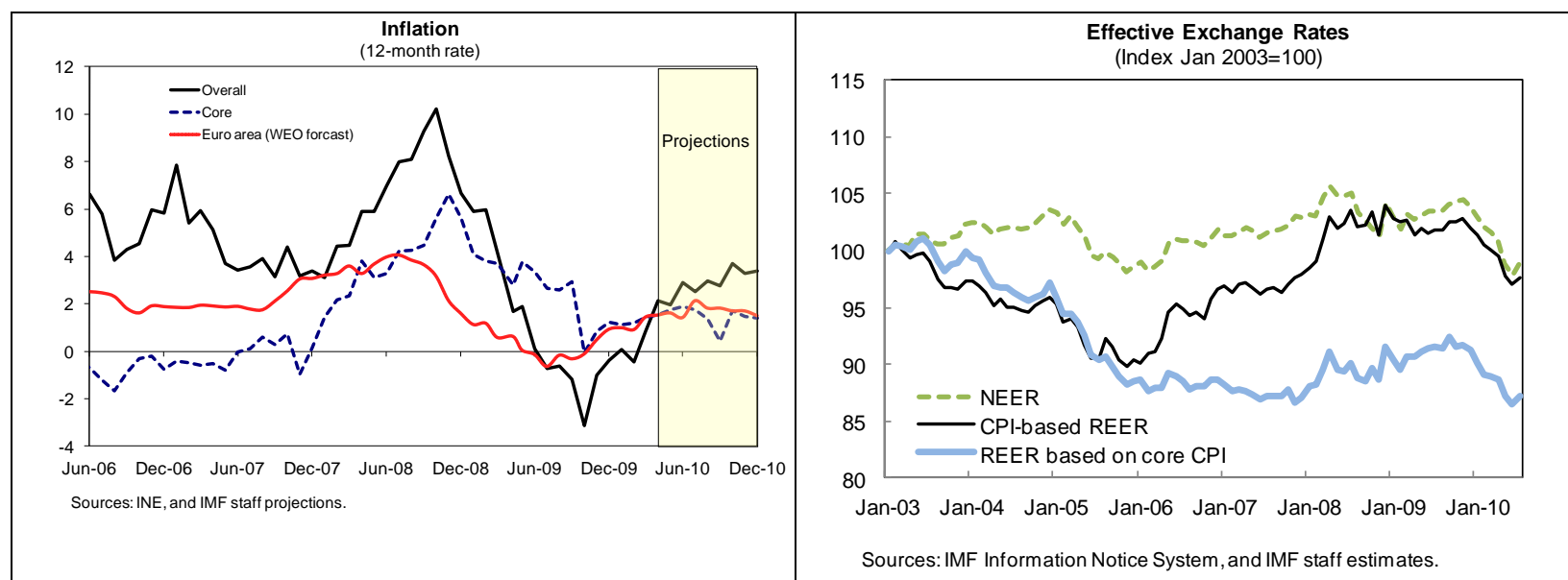

5. International trade and financial inflows are improving in the first half of $\mathbf{2 0 1 0 .}$

Merchandise imports grew by approximately 5 percent when compared to the same period of the previous year. Service exports grew modestly, but hotel operators are reporting high advanced booking rates through the high season in the remainder of the year. Merchandise exports have achieved substantial growth (around 70 percent, although from a small base), mainly driven by fishery products. In the financial account, FDI has stabilized and strong pace of official disbursements should contribute to a positive overall balance of payments in 2010 . 


\section{Box 2. Assessment of the Exchange Rate}

Cape Verde has a fixed exchange rate regime, with a peg to the euro. The competitiveness of the escudo was assessed using three alternative approaches based on the IMF's methodology, adapted for Cape Verde's specific circumstances, and using medium-term fundamentals (projections for 2014). ${ }^{3}$ Although the analysis has a high degree of uncertainty and is sensitive to methodological differences, the evidence shows that the escudo is broadly aligned with its fundamentals.

Results of CGER-type Analysis

\begin{tabular}{lccc}
\hline & ERER & MB & ES \\
\cline { 2 - 4 } Current account norm (percent of GDP) & $\ldots$ & -7.3 & -8.1 \\
Current account gap (percent of GDP) & $\ldots$ & -2.4 & -1.6 \\
Misalignment (+ overvaluation, percent) & -12.5 & 9.0 & 6.2 \\
\hline Source: IMF staff estimates and projections & &
\end{tabular}

The equilibrium real exchange rate approach (ERER) estimates a reduced-form relationship between the REER and macroeconomic fundamentals (e.g. relative productivity as proxied by GDP at a purchasing power parity exchange rate per unit of labor; terms of trade; government consumption to GDP; Net Foreign Asset (NFA); aid inflows; and remittances). The ERER suggests the exchange rate is undervalued by about 12.5 percent.

The macro-balance approach (MB) estimates an equilibrium current account balance (i.e. norm) as function of macroeconomic fundamentals (e.g. relative old age dependency; relative population growth; relative income; relative income growth; relative fiscal balance; oil trade balance; initial NFA; aid inflows; and remittances) and estimates the change in the exchange rate necessary to adjust the underlying current account to the norm. This approach suggests a small gap of -2.4 percent of GDP between the current account norm and the underlying current account in 2014, implying an exchange rate overvaluation of 9 percent. Large negative current account norm is due to low level of NFA and relatively young population, which reduce national savings and hence current account balance.

The external sustainability approach (ES) computes the current account needed to stabilize the net foreign position at a benchmark level, and then estimates the exchange rate change required to adjust the underlying current account to this norm. Based on the medium-term average projections for real growth, the ES suggests a small gap of -1.6 percent of GDP, implying an exchange rate overvaluation of 6.2 percent.

An appropriate assessment of Cape Verde's external competitiveness also needs to take into account the country's main export industry: tourism. During the depths of the global financial crisis, the tourist industry demonstrated its considerable price flexibility as hotels slashed prices to adjust to the demand shock and, in doing so, maintained solid growth in number of hotel nights. Cape Verde's export growth potential in the tourist sector hinges on alleviating bottlenecks in supporting infrastructure, particularly ports, airports, electricity, and water.

\footnotetext{
${ }^{3}$ The exchange rate assessment used a template and dataset by Burcu Aydin (2010), IMF working paper 10/162, which extends the CGER methodology to include a few fundamentals specific to Africa.
} 


\section{B. Risks to the Outlook}

6. Risks to the outlook are broadly balanced. Growth is projected to pick up in 2010 and gather momentum over the medium term as global conditions improve. Private capital inflows, particularly FDI, are expected to improve, but the current account balance will likely decline significantly in 2010-11 due to the high import content of the PIP. The PIP should nonetheless support medium-term growth, with a significant upside potential for crowding in private sector investment, especially in the tourist industry. However, Cape Verde has strong links to Europe through official lending, tourism, FDI, and remittances. Thus, the deterioration of European conditions in recent months has amplified downside risks, and austerity measures in donor countries could have some negative effects on tourism as well as disbursements and approval of new projects.

\section{Policy Discussions: Rebuilding BufFers AND DeVeloping Markets}

\section{A. Improving Infrastructure While Maintaining Fiscal Sustainability}

\section{Accelerating the public investment program}

\section{Large fiscal and current account deficits over 2009-12 reflect a temporary} acceleration of the public investment program as counter-cyclical stimulus. The authorities have taken advantage of a transitional window for concessional external financing following graduation to middle-income status and the need for countercyclical stimulus to accelerate their PIP. ${ }^{4}$ The PIP aims to alleviate infrastructure bottlenecks, including those that hinder development of the tourism sector, and to support progress on social policies. The IMF has supported this strategy, which is contributing to a temporary deterioration of fiscal balances relative to those planned before the crisis (e.g., the fifth PSI review in 2008). ${ }^{5}$ Likewise, the large temporary current account deficits reflect higher imports associated with public investment. Despite this temporary deterioration of fiscal and external balances, Cape Verde's macroeconomic fundamentals are broadly stable and sustainable, and meet the PSI requirements: (i) the large fiscal and current account deficits are financed by one-off concessional resources; (ii) absent any capital spending financed externally (through loans and grants), Cape Verde would realize fiscal surpluses and small current account deficits, in line with PSI requirements, and would compare favorably with other PSI cases; (iii) the feasibility study show that the projects should bring significant value added and revenues in the medium term.

\footnotetext{
${ }^{4}$ With Cape Verde's graduation from least-developed-country status, donors have provided a transitional period for concessional funding over several years. The authorities are using this window of opportunity to finance as much as possible of the infrastructure investment needs on favorable terms. The World Bank and outside consultants have appraised large projects.

${ }^{5}$ The deterioration also reflects lower revenue associated with the economic slowdown. Domestic revenue is approximately 1/2 percent of GDP lower in 2010-11 in the current projections relative to projections from the fifth review in 2008, and is only partially offset by lower domestic expenditures.
} 
8. The authorities are confident of a high rate of project implementation in 2010. Preliminary data suggest an execution rate of public investment of 46 percent at end-August. The authorities expect the implementation rate to accelerate through the remainder of the year and they project capital spending at 22.6 percent of GDP for 2010.

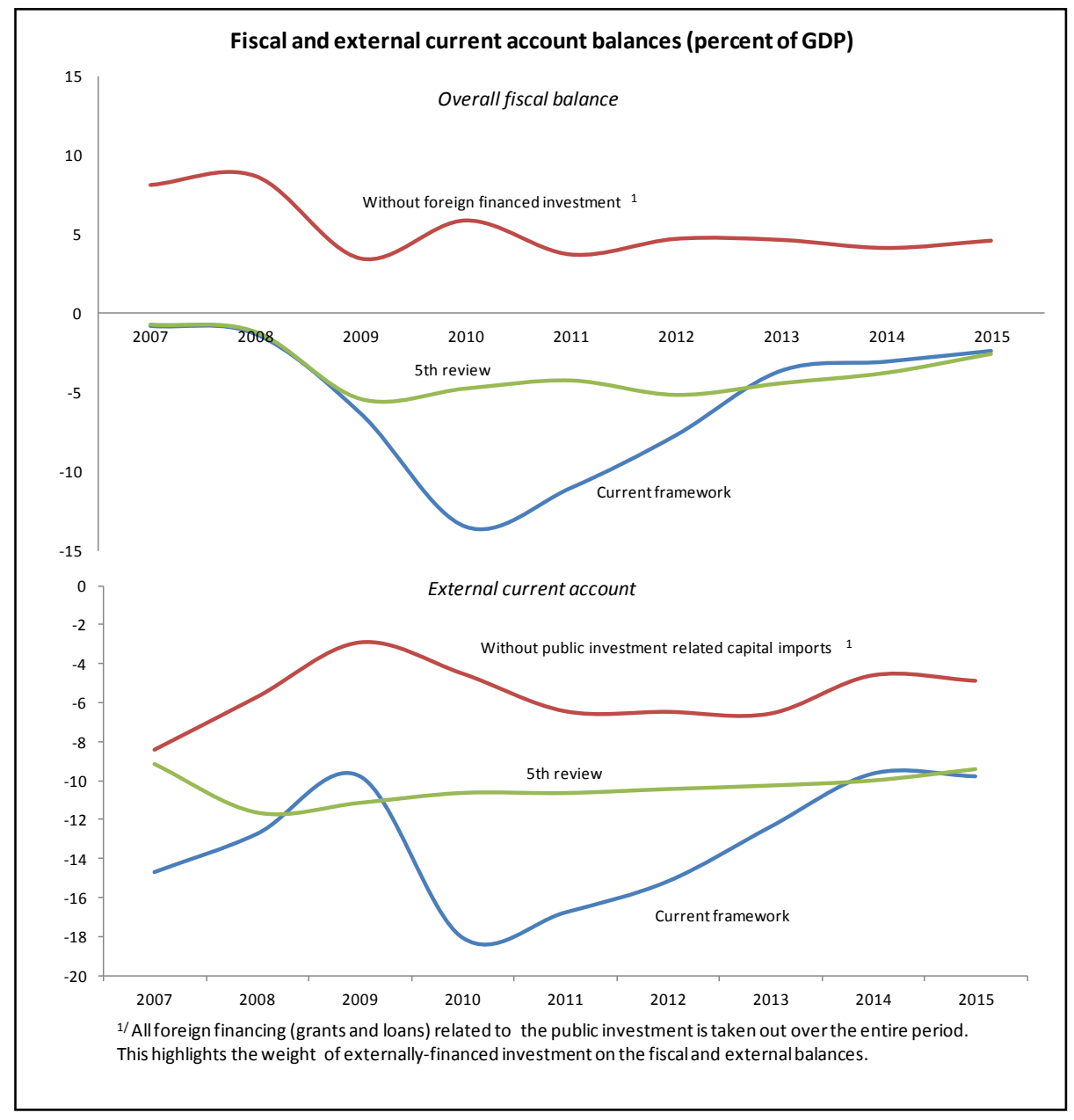

9. The public investment program aims to achieve national development objectives for increasing growth, diversifying the economy, and protecting vulnerable groups. The authorities have completed cost-benefit assessments for all projects, most often in partnership with donors or lenders. Staff supports the objectives of the investment program, noting that many of the projects should enhance competitiveness by relieving infrastructure bottlenecks that act as a cost to business. Staff nonetheless underscores the need for judicious selection and transparent implementation of projects. Thus, staff advised the authorities to exercise caution in agreeing to foreign loans with procurement requirements tied to the donor in order to minimize costs and to crowd in domestic provision from the private sector.

\section{Maintaining external debt sustainability}

10. The authorities are cognizant of the need to manage external debt well and safeguard debt sustainability (MEFP II 5). They are committed to taking advantage of their 
window of opportunity for obtaining concessional financing for the public investment program. Indeed, with low interest rates and long grace periods and maturities, debt service ratios remain very low, minimizing liquidity and solvency risks. The rapid implementation of the PIP during 2009-12 would attenuate the impact of the global slowdown and would provide the foundation for sustained medium-term growth, which should enhance repayment capability. Nonetheless, given the prospect of a gradual move to non-concessional financing in the medium run, the authorities wish to make debt management a key element of the new PSI supported program. Staff concurs, but also emphasizes the need to rebuild fiscal policy buffers following the rise in debt stocks relative to before the crisis. Thus, to provide fiscal space for adjusting to future shocks, staff stressed that the authorities should avoid incurring any new foreign borrowing beyond that already planned and included in the current debt sustainability analysis (see DSA supplement). Scaling back spending in the medium-run would also provide breathing space to ensure that the current investment generates the expected growth dividends.

\section{B. Supporting the Exchange Rate Peg}

\section{Keeping domestic debt low}

\section{Fiscal policy contributes to protecting the peg by keeping net domestic debt low} (MEFP I 4). Cape Verde made commendable progress in reducing the stock of net domestic debt below 20 percent of GDP during the 2006-10 PSI. The authorities achieved their objective two years ahead of schedule. Staff projects net domestic debt to stay below 20 percent of GDP in 2010 and 2011, but the debt ratio increases slightly. Staff agreed with the need to keep net domestic debt low, and advised resisting pressure on domestic borrowing as external financing is scaled back over the medium term.

\section{Faced with weak revenue, the government has been holding current expenditure} below budget in 2010 to minimize the need for domestic borrowing. Despite an acceleration in VAT collection, tax revenues have been below budget through August, reflecting weaker activity than foreseen at the time of the MTFF and 2010 budget preparation. In response, the authorities have stepped up the collection of tax arrears (MEFP II 6). They also took bold measures to curb current spending - despite pressures during an election year-including by freezing new hiring in the public sector. The authorities have held down spending on wages and salaries, and domestically financed capital spending, but they have protected social spending such as pensions and subsidies to the poor. In the remaining months of the year, domestic revenues are expected to accelerate slightly and additional budget support from the European Union should contribute to higher grants. 


\begin{tabular}{|c|c|c|c|c|}
\hline \multicolumn{5}{|c|}{ Text Table: 2010 Fiscal Developments (percent of GDP) } \\
\hline & \multicolumn{4}{|c|}{2010} \\
\hline & & & Proj. & Budg. \\
\hline & Q1 ${ }^{1 /}$ & August ${ }^{1 /}$ & Dec. & Dec. \\
\hline Domestic revenues & 20.7 & 20.8 & 23.1 & 24.7 \\
\hline o/w VAT & 6.8 & 7.5 & 7.9 & 8.5 \\
\hline $\mathrm{o} / \mathrm{w}$ Income Tax & 6.8 & 5.5 & 5.9 & 6.6 \\
\hline Grants & 3.0 & 3.3 & 7.1 & 6.2 \\
\hline Current expenditure & 17.2 & 18.6 & 21.0 & 21.1 \\
\hline $\mathrm{o} / \mathrm{w}$ wage bill (without pensions) & 8.9 & 9.4 & 10.3 & 10.7 \\
\hline $\mathrm{o} / \mathrm{w}$ transfers and subsidies & 4.8 & 5.3 & 5.4 & 5.8 \\
\hline Investment expenditure & 8.3 & 14.6 & 22.6 & 20.4 \\
\hline Overall balance & -1.8 & -9.0 & -13.5 & -10.6 \\
\hline \multicolumn{5}{|c|}{$\begin{array}{l}\text { Source : Cape Verde authorities and staff calculations. } \\
1 / \text { GDP is measured at the equivalent fraction of the year. }\end{array}$} \\
\hline
\end{tabular}

\section{Building and stabilizing reserves}

\section{Monetary policy is geared toward} maintaining the euro peg. The $\mathrm{BCV}$ raised its policy rate during the international crisis reflecting heightened global risk premia. In spite of the severe shock, foreign reserves fell only marginally and the peg held firm. Global risk indicators (such as the EMBI spread) subsequently dropped, but the BCV has been cautious in its response. It reduced its policy rate only in January 2010 by one percentage

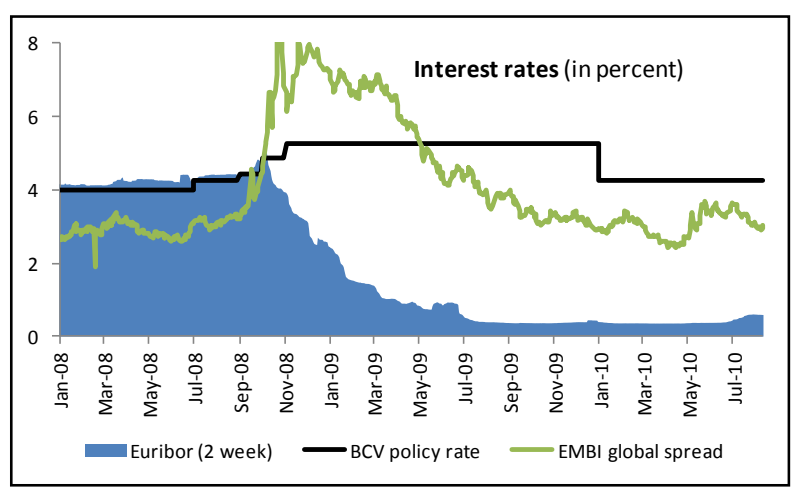
point to 4.25 , and the spread with the Euribor remains large.

\section{To support the peg, monetary policy focuses on ensuring gradual reserve} accumulation (MEFP II 9). The BCV has set a high minimum reserve requirement and a policy rate well above Euribor. The authorities view this restrictive monetary stance as necessary to encourage inflows of emigrant deposits, which respond gradually and at long and variable lags to interest rates, and to restrain private sector credit that would fuel imports. They are cautious about lowering the policy rate and reserve requirements until foreign reserve accumulation is definite and the global environment rebounds. ${ }^{6}$ Staff supports the BCV's cautious monetary stance given the benefit of further building foreign reserve buffers and the current uncertain global environment. Staff also agreed to support efforts to better understand the monetary transmission mechanism, particularly the impact of interest rate changes on emigrant deposits and foreign reserves, to improve policy effectiveness. Staff advised to closely monitor the

\footnotetext{
${ }^{6}$ An MCM mission will visit Cape Verde in November 2010 to provide TA in this area.
} 
composition of capital flows and adopt macro-prudential regulations to minimize risks associated with interest-rate sensitive inflows. To reduce distortions to financial intermediation, staff also recommended to reduce the minimum reserve requirement or allow treasury securities to satisfy part of the requirement.

\section{The BCV considers their monetary framework to be procyclical. An economic} slowdown in Cape Verde's main trading partners in Europe would call for a tighter monetary stance at home to offset the impact of sluggish export growth on the balance of payments and reserves. Thus, even though limited capital mobility may provide some scope for independent monetary policy, the BCV cannot exploit it given the required focus on stabilizing the balance of payments. While agreeing that a larger reserve buffer should be accumulated at the rate of 0.1 month of imports per year, staff argued that prudent fiscal policies should be the main instrument for reserve accumulation.

\section{Safeguarding Financial Stability and Developing Markets}

\section{Though the onshore banking sector remains sound, the crisis spawned some}

vulnerabilities. The overall capital adequacy ratio of onshore banks stood at about 12 percent at end-June 2010 (Table 8). Two banks fell below the minimum regulatory capital requirements, due to connected lending and off-balance sheet transactions. Asset quality and profitability sharply deteriorated because of spillover effects from the global crisis, which affected foreigners' second-home real estate market and the construction sector. ${ }^{7}$ Higher provisions also contributed to the decline in profitability. However, funding held up well. Emigrant deposits — which constitute about 37 percent of the total banking sector deposits and originate predominately from Portugal, France, and the United States - remained steady and grew by over 5 percent (y-o-y). Although the first and third largest domestic banks are majority-owned by a Portuguese state bank, which had a favorable result in the recent European Union stress tests, parent funding of domestic banks is relatively small.

17. The authorities are making progress to safeguard financial stability (MEFP II 12-13). The draft new banking law, under discussion by the council of ministers, would bring all banks under a single banking law; thereby leveling the playing field and increasing competitiveness within the sector. The BCV is in the process of producing the first financial stability report on an annual basis. The regulatory and supervisory framework is being improved by: (i) establishing a macroprudential unit within the $\mathrm{BCV}$; (ii) moving gradually toward risk-based supervision; (iii) enhancing both the off-site and on-site supervision procedures; (iv) strengthening the skills of the banking supervision staff; (v) developing prudential regulations on interest rate, foreign exchange, and credit risks; and (vi) strengthening IT systems to facilitate effective supervision. Staff supported the BCV's plan to expand and formalize the standing financial stability committee to include the Ministry of Finance $(\mathrm{MoF})$ in a memorandum of understanding (MoU).

\footnotetext{
${ }^{7}$ NPLs should be interpreted with caution since a loan is considered nonperforming if it is only one day overdue. For instance, as of May 2010, NPLs of one of the commercial banks would decline from 7.6 percent to 4 percent if restated according to the common practice of 90 days overdue.
} 
The MoU should set out an action plan to distribute duties for financial stability between the $\mathrm{BCV}$ and MoF and develop an effective public communication strategy.

18. Staff urged the BCV to continue to enhance banking supervision. The short-lived episodes of non-compliance with the regulatory capital requirements are a source of concern. ${ }^{8}$ One bank was marginally under-capitalized, while another bank (with relatively small market share) was under corrective actions. The BCV should enhance its supervisory framework to allow for detection of potential banks weaknesses and take corrective actions at an early stage. A deeper understanding of each bank's strategy and business model should complement current compliance-based supervision. Additionally, with high interest rates, onshore banks have started attracting non-resident deposits, which are exempt from reserve requirements. The authorities and staff agreed on imposing a punitive minimum reserve requirement and adopting prudential regulations to minimize any destabilizing capital inflows. ${ }^{9}$

\section{The authorities continue to develop primary and secondary markets for government} securities, and enhance monetary operations (MEFP \& 10-11, Box 3). Issuing fungible securities at a uniform price in the primary market and publishing the auction calendar well in advance are important steps for fostering a liquid debt market. Staff also supports the authorities' fiscal-monetary coordination agreement to use treasury securities as the main instrument of monetary policy, and thereby enhance the effectiveness of the monetary transmission mechanism. Furthermore, as the National Social Security Institute (INPS) dominates and distorts the primary market for government securities, staff urged the authorities to improve the process of auction assignment by allowing the INPS to participate in the non-competitive group. Equally important, the INPS should finalize its investment policy guidance, including investing some of its portfolio abroad, and set up an investment committee.

\footnotetext{
${ }^{8}$ BCV's recognition of these lapses was impeded by the transition to the International Financial Reporting Standards (IFRS), which was completed only in June.

${ }^{9}$ Emigrant deposits are classified as resident deposits.
} 


\section{Box 3. Liquidity Management and Monetary Operations}

Although the fixed exchange rate regime limits the room for conducting independent monetary policy, the BCV can still play a role in liquidity management. High and volatile excess liquidity in the banking sector has complicated liquidity management, and discourages interbank transactions. The BCV has relied on open-market operations to sterilize excess liquidity using BCV bills, and has set high reserve requirements (16 percent of deposits).

\section{A liquid debt market and market-based instruments could facilitate liquidity} management and monetary operations. Government bonds are still not fungible, and the primary market is dominated by the INPS, thereby creating market fragmentation and limiting liquidity in the secondary market. The recent reforms to improve the government primary market and use the treasury securities as the main instrument for monetary operations (MEFP II 10 and 13) will help foster a liquid debt market. In addition, a sound liquidity management framework will encourage financial market development and enhance the monetary transmission mechanism. To accomplish this, the deposit and lending facilities at the BCV could be improved by allowing repo and reverse repo operations (using treasury securities) to absorb and inject liquidity in the financial system. The interest rates on those facilities would set a corridor on market interest rates. Additionally, the BCV could consider allowing treasury securities to satisfy part of the required reserves and/or remunerate required reserves.

\section{Reducing Fiscal Risk in State-Owned Enterprises}

20. The fiscal risk arising from state owned enterprises is still a challenge that needs to be addressed (MEFP $\mathbb{I}$ 7). The government has undertaken reforms to the loss-making public electricity and airline companies, but with no drastic improvement in profitability. The recent Law of State Enterprises and Public Managers provides a framework for better management of SOEs. To reap the benefit of the framework, the authorities plan to introduce and enforce managerial incentive contracts linked to enterprise performance. Short-term efforts will also focus on improving operational and commercial efficiency within the firms. ${ }^{10}$ The staff urged authorities to move forward with this reform agenda. Staff considered that private sector involvement can also help to restore profitability, as long as public-private contracts are well designed and appropriately share costs and risks.

\section{E. Social Policies to Protect the Vulnerable}

\section{PSI-supported policies contributed to the strong record of social development in} Cape Verde (Box 4). Cape Verdean authorities have a long-standing commitment to social development. The Poverty Reduction Strategy documents have reaffirmed this commitment by pursuing a multi-pronged development approach to address priority areas such as social cohesion and human development (MEFP II 8). ${ }^{11}$ The authorities emphasized that macroeconomic stability

\footnotetext{
${ }^{10}$ The World Bank is actively engaged on this area of reform through its budget support operations.

${ }^{11}$ Cape Verde's annual progress report on the implementation of its PRSP in 2009 was finalized in April 2010 and is expected to be submitted to the Board before the first review.
} 
is at the core of the country's multi-pillar development strategy. As a result, PSI-supported policies continue to play an important role in the overall reform framework.

\section{F. Sustaining Medium-term Growth}

\section{The PIP is central to sustaining medium-term growth in Cape Verde not only} because of its overall reach and size, but also due to its relevance at the project-level. The PIP specifically addresses fundamental infrastructure bottlenecks to economic activity in Cape Verde. These include: insufficient wharf space for vessels, inadequate port equipment for cargo handling, deficient airport facilities across islands, lacking logistics to support intermodal transportation, underinvestment in energy generation and water production. Tackling such issues is expected to reduce costs to private sector investment, boosting the country competitiveness and growth prospects. ${ }^{12}$

\section{Over the medium-term, the tourist industry has the potential to act as an engine of} growth. Secular trends are favorable: as global growth rebounds and as increasing numbers of the global population reach retirement age, the tourist industry has the potential to expand faster than other industries. Government efforts to improve infrastructure and private initiatives such as transportation should help reduce constraints in the industry, but challenges still remain.

Additional infrastructure needs are likely to arise, particularly in transportation; education and training will need to be targeted to the skills required in the tourist and other growth industries; and land registration will need to be improved to facilitate reliable and sustainable development zones. Moreover, staff and the authorities agreed on the importance of integrating the tourist industry with the domestic economy to ensure balanced broad-based growth.

\section{Staff and the authorities agreed that policies should further encourage} competitiveness and diversification. The authorities emphasized that their PIP will create conditions for diversifying the economy. Additional regulatory improvements (e.g. in Information and Communications Technologies) could help spur diversification into other potential export industries. Staff view price and wage flexibility as well as inter-island mobility are useful to preserve growth and employment against any negative demand shocks, but consider that existing labor market restrictions may hinder a competitive business environment and deter formal employment growth. While tax incentives may have helped nurture a nascent tourist industry, the objective over the medium-run should be to create a level playing field with all industries contributing efficiently and equitably to finance development.

\footnotetext{
12 "The average annual GDP growth rate in Cape Verde during the decade before the global crisis (1998-2007) was 7.3 percent. The main sectors driving growth were construction, tourism, transportation and commerce, fueled by strong investment and consumption growth coming from the private sector. The PIP will boost supply by further increasing returns from private sector investment in the medium-run while contributing to stronger aggregate demand in the more immediate future as the economy recovers. Annual GDP growth is projected to average 5.9 percent in the post-crisis period (2010-2020), in line with long-term historical growth rates.".
} 


\section{Box 4. Cape Verde's Social Policies}

In 2010, Cape Verde has made substantial progress toward accomplishing its MDG targets. Social development has been noteworthy and has matched the economic transition to middle-income status.

\begin{tabular}{|lcccc|}
\hline & $\begin{array}{c}\text { Cape Verde } \\
\text { GDP per capita, PPP (constant 2005) }\end{array}$ & $\begin{array}{c}\text { Cape Verde } \\
\text { 2000 }\end{array}$ & $\begin{array}{c}\text { Lower middle } \\
\text { income 2008 }\end{array}$ & $\begin{array}{c}\text { Sub-Saharan } \\
\text { Africa 2008 }\end{array}$ \\
Poverty (\%) & 2272 & 3309 & 4082 & 1938 \\
Literacy rate (\%) & 39 & 26 &.. &.. \\
Literacy rate - 15 to 24 yrs (\%) & 75 & $>80$ & 80 & 63 \\
Child mortality - less 5 yrs (per 1000) &.. & 98 & 89 & 72 \\
Maternal mortality (per 100000) & 39 & 20.1 &.. &.. \\
Access to improved water source (\%) & 86.3 & 53.7 & 230 & 645 \\
Measles vaccination - less 1yr (\%) & 72.8 & 84 & 86 & 60 \\
\hline
\end{tabular}

Source: Government of Cape Verde and World Development Indicators

Social policies have focused on fostering labor market participation and providing direct assistance to vulnerable groups (unprivileged children, poor elderly individuals, females who are head of households, those in chronic poverty and with disabilities). Some key social programs include:

Education. Policy has focused on improving quality, ensuring equity, and achieving measurable results. A gradual decentralization in the management of programs has also contributed to the recent strong record: Elementary school is mandatory for six years and net enrollment rate increased from 53.9 percent in 2001 to 62 percent in 2008. Transition rates from elementary to the next school cycle increased from 70.7 percent in 2001 to 82.9 percent in 2008 . Recently, local universities have been constructed and will allow youth the option of pursuing undergraduate studies in the country.

Health. Policy aims to achieve an accessible and encompassing coverage for treatment and preventive care. The National Health Services is being re-organized to guarantee that the strong performance of health indicators are maintained and continue to support human capital development. Additionally, the country continues to build capacity for public health surveillance to deal with risks such as dengue fever and other diseases.

Job creation and empowerment. A number of initiatives target the youth, who have high rates of unemployment. The objective is to create an integrated system linking general education, professional training and employment, through (i) active labor market policies such as apprenticeship, internship and first-job programs, and (ii) private sector involvement in training. The initiatives help match training with labor demand and allow youth to directly benefit from the development process.

School feeding program. This internationally-recognized program provides free meals to nearly 80,000 children in the country, contributing to improved nutrition and human capital development.

Social pension. Non-contributive pension payments are available to those in need. The government, with support from municipalities and the civil society, screen and identify candidates for the program.

Gender equality. Implementation of public policies to democratize access to education across gender has led Cape Verde to achieve gender parity in primary and secondary education.

Water, sanitation and social housing. The government is carrying out public investment to guarantee access to basic public services and decent living conditions. 


\section{Program Monitoring AND Risks}

\section{A. Program Monitoring}

25. For monitoring performance under the program, quantitative assessment criteria, quantitative indicative targets, and structural benchmarks have been proposed (MEFP Tables 1 and 2). The assessment dates will be end-March 2011 and end-September 2011 with corresponding semi-annual reviews. ${ }^{13}$ The quantitative targets include ceilings on net domestic borrowing of the central government, net domestic assets of the BCV, and accumulation of non-concessional external debt; and a floor on net international reserves of the BCV. Targets to be monitored on a continuous basis include ceilings on accumulation of domestic and external arrears, non-accumulation of short-term non-concessional external debt and a floor on the regulatory capital of commercial banks. Structural benchmarks aim at strengthening the tax base, the debt management, the financial sector, the fiscal-monetary coordination, the government securities market, and promoting fiscal accountability.

\section{B. Statistical Issues}

\section{Progress has been made in improving economic and financial statistics, though} further improvements in data are needed. The data are broadly adequate for surveillance. The production-based GDP is compiled by the National Statistical Institute (INE) with long delays, and labor market data on unemployment is published only on an annual basis. The ongoing efforts by the INE to finalize the 2010 census should assist other government agencies in adopting sound policies. Staff agreed that there is a need to strengthen the national accounts, and consider technical assistance in this area as a priority.

\section{Program Risks}

\section{The main program risks consist of renewed adverse external shocks and fiscal} pressures ahead of elections. Worsening economic conditions in Europe could spill over through a decline in tourism, second home purchases of foreigners, FDI, remittances, and official bilateral support, which thereby could weaken the economic recovery and build up of reserves. On the domestic front, the forthcoming elections are expected to be close, creating incentives for a short-term boost in spending. However, the authorities stressed their commitment to holding spending to budget, and have strongly implemented this commitment so far this year.

\footnotetext{
13 The first program review is expected to be completed by July 31, 2011 (rather than the customary six months after approval) to provide sufficient time for review following the March 31, 2011 assessment date.
} 


\section{STAFF APPRAisal}

28. Cape Verde has demonstrated notable economic and policy resilience. In the face of adverse global shocks, the government used its policy buffers to provide macroeconomic stimulus. The economy is now rebounding strongly and is poised for solid growth over the medium term.

29. The authorities should complete the public investment program underway, but limit new external borrowing in order to restore fiscal buffers. The temporary acceleration of the public investment program is appropriate, given the need to improve infrastructure, the availability of funding on concessional terms, and usefulness of countercyclical stimulus. However, foreign-financed spending would need to be scaled back without resorting to new foreign borrowing, to rebuild fiscal buffers against negative shocks. Foreign-financed projects should be selected exclusively on the basis of pro-growth or pro-poor objectives .

30. The peg to the euro continues to serve Cape Verde well. This arrangement provides a stable anchor for economic stability, while allowing some flexibility in the escudo's exchange rate vis-à-vis currency cross rates. Staff analysis shows that the escudo's real effective value against other currencies is broadly in line with its fundamentals.

\section{The government should maintain low domestic debt as the main instrument to} support the peg. A continued low level of domestic borrowing will be required to sustain a gradual accumulation of foreign exchange reserves. Net domestic debt should be kept below 20 percent of GDP, including by tightly controlling recurrent spending, improving tax administration, and rationalizing tax exemptions. Stronger efforts are also required to address fiscal risk arising from a few public enterprises.

\section{The monetary policy stance is appropriately cautious, but the framework could} be improved in the medium term. Given the weak global environment, the BCV's emphasis on shoring up reserve accumulation is well placed. However, a bias toward restrictive monetary policy introduces other risks. Persistent interest rate differentials between Cape Verde and international markets will inevitably invite speculative inflows, increasing vulnerability. In addition, discouraging lending to the private sector can stifle economic diversification and reduce potential growth. Over the medium term, restoring fiscal and reserve buffers will help provide space to counteract shocks and reduce the need for an active monetary policy.

\section{Monetary operations and the monetary transmission mechanism could be} enhanced. A better understanding of how changes to reserve requirements and policy rates impact bank rates and economic activity would help to calibrate policy actions. To reduce inefficiencies in financial intermediation, high minimum reserve requirements should be reduced or treasury securities allowed to satisfy part of the requirement. The BCV should also improve its standing facilities by using repo and reverse repo operations. 
34. The BCV should step up efforts to safeguard the financial system and develop the government securities market. Staff supports the draft new banking law and ongoing efforts to enhance banking supervision. Further efforts are needed to increase staff and training, and strengthen the supervisory capacity for early detection of vulnerabilities. Recent reforms to enhance liquidity in government securities are welcome. Nonetheless, the authorities should reduce the distortion created by the INPS in the government primary market by including INPS in the non-competitive group for auctions and diversifying the INPS's investment portfolio.

35. While data provision is broadly adequate for surveillance, the authorities are encouraged to continue to improve economic and financial statistics in order to facilitate better monitoring and analysis of developments to guide policy formulation.

36. Staff recommends approval of Cape Verde's request for a new 15-month PSI. The agreed program will build on the macroeconomic success and structural reforms of the previous PSI supported program, and will help maintain macroeconomic discipline through the forthcoming election period.

37. Risks to the program appear manageable. The authorities have demonstrated their ability to preserve macroeconomic control and stability in the face of election pressures and adverse economic shocks. Staff is confident of their continued commitment to program implementation.

38. Staff recommends that the next Article IV consultation take place within 24 months, in accordance with the decision on consultation cycles in program countries. 
Figure 1. Cape Verde: Fiscal Performance

(Percent of GDP)


The economic slowdown reduced tax revenue from 2009.



Net domestic debt will continue to be low and support a gradual rise in gross international

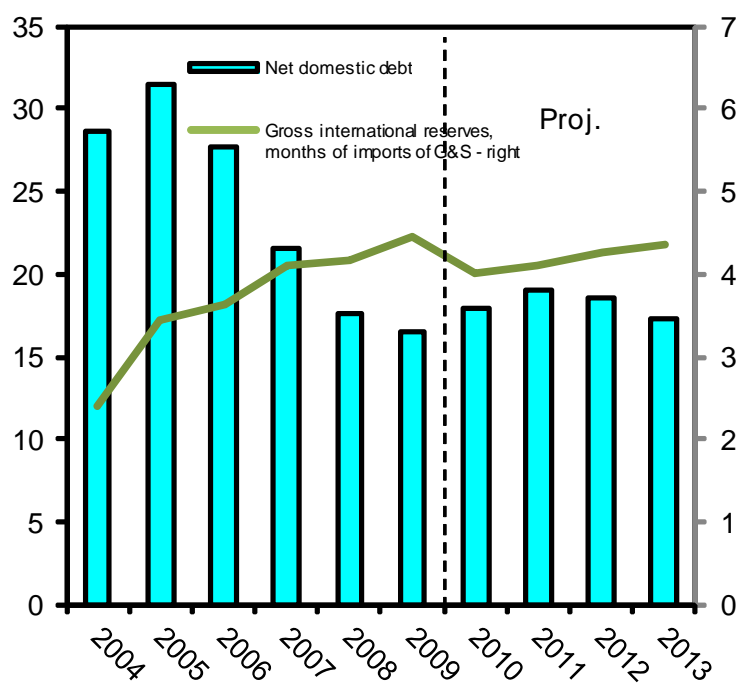

Sources: Cape Verdean auth orities and IMF staff estimates. 
Figure 2. Cape Verde: Monetary Developments.
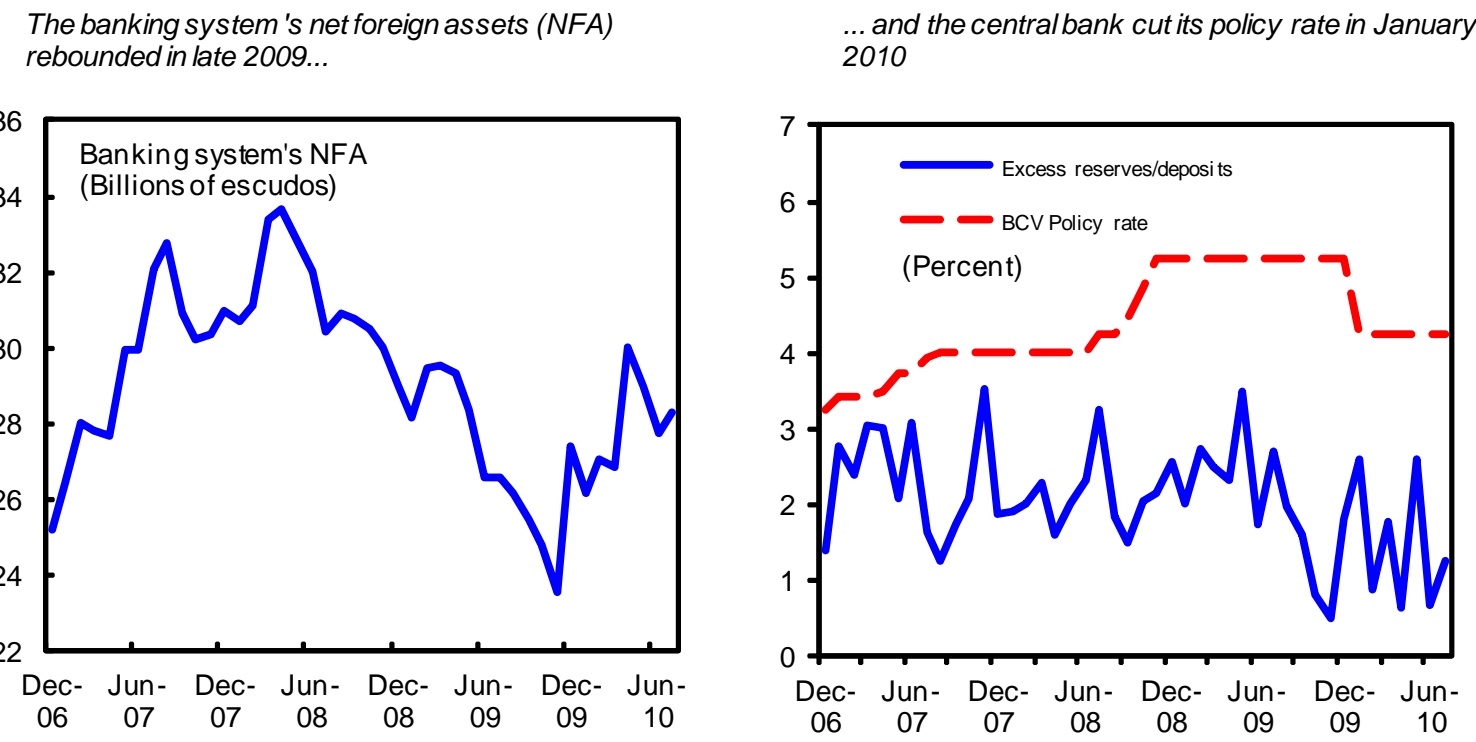

The growth of credit to the private sector has stabilized...


Source: Bank of Cape Verde. 
Figure 3. Cape Verde: External Sector Developments.
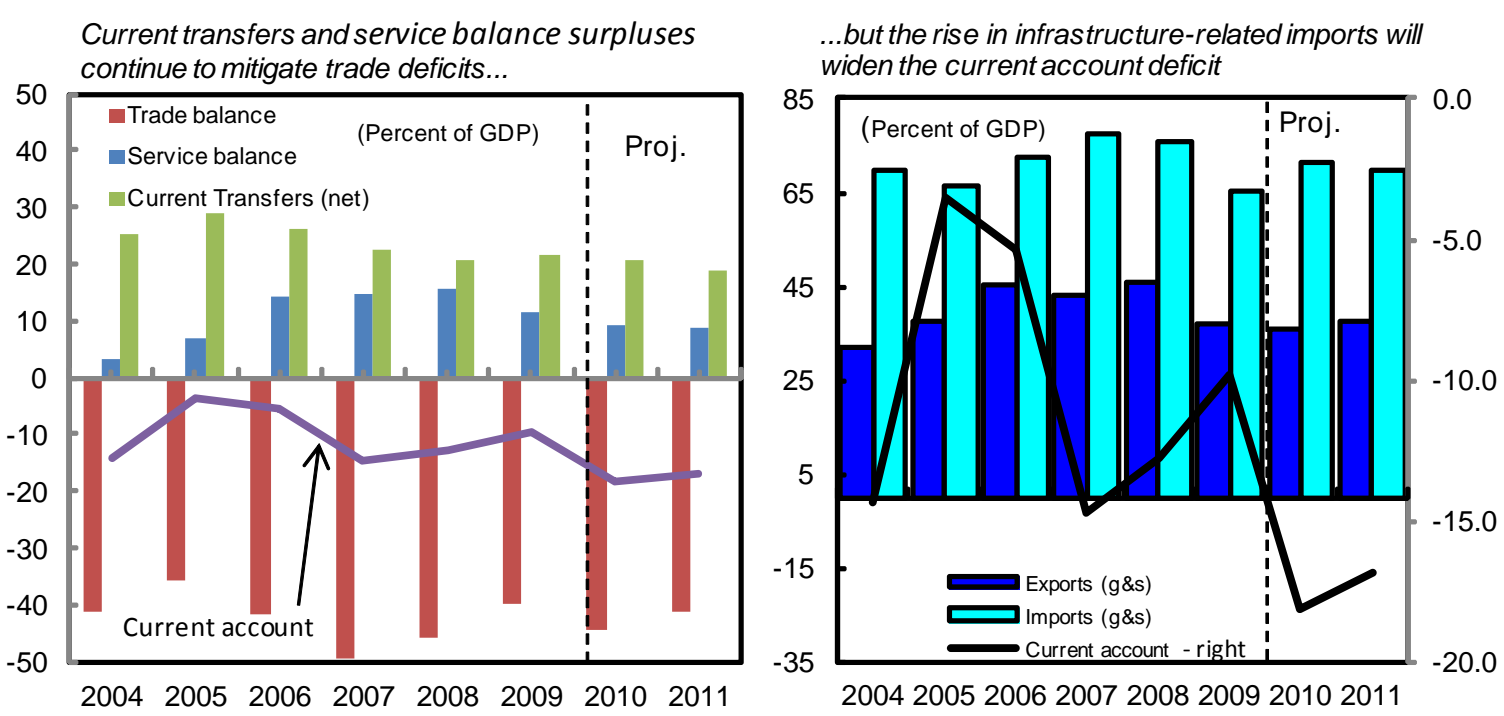

FDI and concessional loans will finance most of the current account deficit...
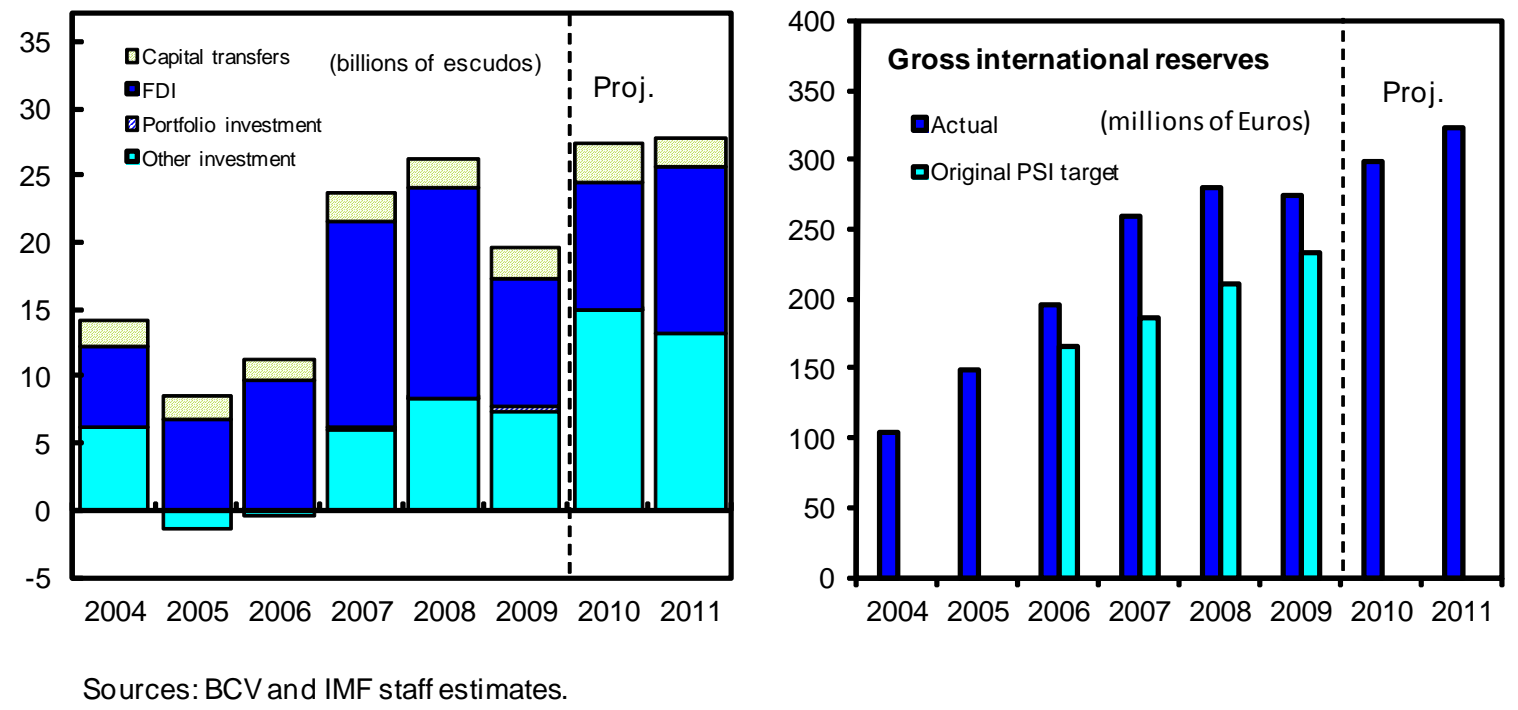

Sources: BCV and IMF staff estimates. 
Table 1. Cape Verde: Selected Economic and Financial Indicators, 2007-14

\begin{tabular}{|c|c|c|c|c|c|c|c|c|}
\hline & 2007 & 2008 & \multirow{2}{*}{$\frac{2009}{\text { Estimates }}$} & 2010 & 2011 & 2012 & 2013 & 2014 \\
\hline & & & & \multicolumn{3}{|c|}{ Projections } & & \\
\hline & \multicolumn{7}{|c|}{ (Annual percentage change) } & \\
\hline \multicolumn{9}{|l|}{ National accounts and prices } \\
\hline Real GDP & 8.6 & 6.2 & 3.6 & 5.4 & 5.9 & 6.8 & 7.6 & 6.9 \\
\hline Real GDP per capita & 7.1 & 4.7 & 0.7 & 3.4 & 2.9 & 3.8 & 4.7 & 3.9 \\
\hline GDP deflator & 1.4 & 2.5 & 4.2 & 3.7 & 3.2 & 2.7 & 2.7 & 2.7 \\
\hline Consumer price index (annual average) & 4.4 & 6.8 & 1.0 & 2.2 & 3.0 & 2.5 & 2.0 & 2.0 \\
\hline \multicolumn{9}{|l|}{ External sector } \\
\hline Exports of goods and services & 4.5 & 16.2 & -13.2 & 6.8 & 12.4 & 15.5 & 15.8 & 17.1 \\
\hline Of which: tourism & 23.0 & 7.9 & -16.2 & 3.9 & 11.8 & 17.5 & 17.5 & 19.3 \\
\hline Imports of goods and services & 17.8 & 6.3 & -7.5 & 19.9 & 5.8 & 7.3 & 7.8 & 8.2 \\
\hline Exports of goods and services - Volume & 13.3 & 4.8 & -10.1 & 2.4 & 7.3 & 13.3 & 14.2 & 15.5 \\
\hline Imports of goods and services - Volume & 18.2 & 2.7 & -4.1 & 23.7 & 0.2 & 4.2 & 8.1 & 9.8 \\
\hline Real effective exchange rate (annual average) & 2.6 & 4.3 & 0.4 & $\ldots$ & $\ldots$ & $\ldots$ & $\ldots$ & $\ldots$ \\
\hline \multicolumn{9}{|l|}{ Government finance } \\
\hline Total revenue (excluding grants) & 17.0 & 10.8 & -9.4 & 6.6 & 11.4 & 10.0 & 9.1 & 15.6 \\
\hline Total expenditure & -0.7 & 13.1 & 9.3 & 34.4 & -1.1 & -0.3 & -4.8 & 9.6 \\
\hline Noncapital expenditure & -0.4 & 3.0 & 9.1 & 7.9 & 10.8 & 4.6 & 4.9 & 10.1 \\
\hline Capital expenditure & -1.4 & 32.9 & 9.6 & 74.0 & -12.2 & -6.1 & -17.4 & 8.6 \\
\hline \multicolumn{9}{|l|}{ Money and credit } \\
\hline Net foreign assets & 23.0 & -6.4 & -5.4 & 9.2 & 12.2 & 12.9 & 10.4 & 19.1 \\
\hline Net domestic assets & 5.6 & 14.4 & 7.0 & 7.5 & 10.0 & 8.6 & 10.5 & 6.6 \\
\hline Of which: net claims on the central government & -61.6 & 31.4 & -6.6 & 40.0 & 16.5 & -1.8 & 2.6 & -17.7 \\
\hline credit to the economy & 25.5 & 24.9 & 11.5 & 10.8 & 12.1 & 11.1 & 13.7 & 11.3 \\
\hline Broad money (M2) & 10.8 & 7.6 & 3.5 & 8.0 & 10.6 & 9.8 & 10.5 & 10.0 \\
\hline Income velocity (GDP/M2) & 1.19 & 1.19 & 1.22 & 1.21 & 1.19 & 1.19 & 1.19 & 1.19 \\
\hline \multirow[t]{2}{*}{ Reserve money (M0) } & 10.0 & 8.0 & 3.4 & 4.6 & 9.3 & 8.8 & 9.9 & 9.5 \\
\hline & \multicolumn{8}{|c|}{ (Percent of GDP, unless otherwise indicated) } \\
\hline \multicolumn{9}{|l|}{ Saving-investment balance } \\
\hline Gross capital formation & 46.4 & 46.6 & 39.4 & 49.6 & 46.1 & 43.5 & 39.0 & 38.0 \\
\hline Government & 11.4 & 13.9 & 14.1 & 22.6 & 18.3 & 15.6 & 11.7 & 11.5 \\
\hline Nongovernment & 35.0 & 32.6 & 25.3 & 27.0 & 27.8 & 27.9 & 27.4 & 26.5 \\
\hline Gross national savings & 32.3 & 33.8 & 29.6 & 31.5 & 29.3 & 28.3 & 26.6 & 28.3 \\
\hline Government & 11.1 & 14.3 & 11.3 & 8.8 & 6.8 & 7.4 & 7.5 & 7.9 \\
\hline Nongovernment & 21.2 & 19.5 & 18.4 & 22.7 & 22.5 & 20.9 & 19.1 & 20.4 \\
\hline External current account (including official current transfers) & -14.7 & -12.8 & -9.8 & -18.1 & -16.8 & -15.2 & -12.4 & -9.7 \\
\hline External current account (excluding official current transfers) & -19.8 & -19.5 & -17.3 & -25.2 & -21.8 & -19.8 & -16.4 & -12.9 \\
\hline Overall balance of payments & 6.6 & 1.7 & -1.0 & 1.9 & 1.8 & 2.5 & 2.1 & 4.0 \\
\hline \multicolumn{9}{|l|}{ Government finance } \\
\hline Total domestic revenue & 27.6 & 28.1 & 23.6 & 23.1 & 23.6 & 23.7 & 23.4 & 24.6 \\
\hline Total external grants & 5.2 & 5.5 & 5.4 & 7.1 & 5.0 & 4.6 & 4.0 & 3.2 \\
\hline Total expenditure & 33.6 & 34.9 & 35.3 & 43.6 & 39.6 & 36.0 & 31.0 & 30.9 \\
\hline Overall balance before grants & -6.0 & -6.8 & -11.8 & -20.5 & -16.0 & -12.3 & -7.6 & -6.3 \\
\hline Overall balance (including grants) & -0.8 & -1.3 & -6.3 & -13.5 & -11.0 & -7.7 & -3.6 & -3.0 \\
\hline Primary basic balance ${ }^{1}$ & 6.1 & 5.8 & 1.2 & 1.8 & 0.4 & 1.6 & 2.1 & 2.0 \\
\hline External financing (net) & 2.2 & 3.0 & 5.0 & 11.8 & 9.3 & 7.4 & 4.0 & 2.7 \\
\hline Domestic financing (net) & -0.8 & -1.0 & 0.8 & 1.6 & 1.7 & 0.3 & -0.3 & 0.4 \\
\hline Public debt & & & & & & & & \\
\hline Total nominal government debt ${ }^{2}$ & 68.0 & 65.0 & 63.9 & 80.9 & 86.7 & 87.7 & 84.2 & 79.5 \\
\hline External government debt ${ }^{3}$ & 46.5 & 47.3 & 47.3 & 63.0 & 67.6 & 69.1 & 66.9 & 63.8 \\
\hline Domestic government debt, net of deposits & 21.5 & 17.7 & 16.5 & 18.0 & 19.1 & 18.5 & 17.3 & 15.7 \\
\hline External debt service (percent of exports of goods and services) & 5.1 & 4.6 & 5.1 & 5.0 & 4.7 & 4.6 & 4.0 & 4.2 \\
\hline Present Value of External Debt - DSA & & & & & & & & \\
\hline PV of debt to GDP (risk threshold: $50 \%$ ) & $\cdots$ & $\ldots$ & $\cdots$ & 43.7 & 47.2 & 48.6 & 47.7 & 47.1 \\
\hline PV of debt-to-revenue ratio ( risk threshold: $300 \%$ ) & $\ldots$ & $\ldots$ & $\ldots$ & 189.6 & 200.1 & 205.2 & 203.9 & 191.7 \\
\hline PV of debt-to-exports ratio ( risk threshold: $200 \%$ ) & $\cdots$ & $\ldots$ & $\ldots$ & 121.2 & 126.7 & 123.8 & 116.0 & 107.7 \\
\hline External current account ( $€$ millions, including official transfers) & -143.1 & -135.0 & -111.8 & -225.4 & -227.9 & -226.4 & -204.1 & -175.1 \\
\hline Gross international reserves (€ millions, end of period) & 259.5 & 280.0 & 275.3 & 298.4 & 322.8 & 360.6 & 395.2 & 467.1 \\
\hline Gross international reserves to reserve money & 1.2 & 1.2 & 1.2 & 1.2 & 1.2 & 1.3 & 1.3 & 1.3 \\
\hline Gross international reserves (months of current imports of goods and services) & 4.1 & 4.2 & 4.4 & 4.0 & 4.1 & 4.3 & 4.3 & 4.7 \\
\hline Memorandum items: & & & & & & & & \\
\hline Nominal GDP (billions of Cape Verde escudos) & 107.3 & 116.7 & 126.0 & 137.2 & 149.2 & 163.8 & 181.0 & 199.1 \\
\hline Net domestic borrowing, net of arrears clearance & -5.4 & -2.2 & 0.2 & 2.9 & 2.5 & 1.2 & 0.5 & 0.0 \\
\hline Exchange rate (Cape Verde escudos per US $\$$ ) & & & & & & & & \\
\hline Period average & 80.4 & 74.9 & 79.2 & $\ldots$ & $\ldots$ & $\ldots$ & $\ldots$ & $\ldots$ \\
\hline End period & 75.6 & 80.9 & 75.5 & $\ldots$ & $\ldots$ & $\ldots$ & $\ldots$ & ... \\
\hline
\end{tabular}

Sources: Cape Verdean authorities, and IMF staff estimates and projections.

${ }^{1}$ Excluding grants, interest payments, and foreign financed investments

${ }^{2}$ Net of central government deposits; including verified stock of domestic and external arrears.

${ }^{3}$ Excluding claims on the offshore Trust Fund. 
Table 2. Cape Verde: Fiscal Operations of the Central Government, 2008-14

(Millions of Cape Verde escudos, unless otherwise indicated)

\begin{tabular}{|c|c|c|c|c|c|c|c|c|c|c|}
\hline & \multirow[t]{2}{*}{2008} & \multicolumn{2}{|c|}{2009} & \multicolumn{3}{|c|}{2010} & \multirow{2}{*}{$\begin{array}{l}2011 \\
\text { Prog. }\end{array}$} & \multirow{2}{*}{$\begin{array}{c}2012 \\
\text { MTFF }^{1} \\
\end{array}$} & \multirow{2}{*}{$\begin{array}{r}2013 \\
\text { MTFF } \\
\end{array}$} & \multirow{2}{*}{$\begin{array}{l}2014 \\
\text { Proj. }\end{array}$} \\
\hline & & Budget & Est. & $\mathrm{H} 1$ & Budget. & Proj. & & & & \\
\hline Revenue, grants, and net lending & 39,187 & 43,639 & 36,540 & 16,568 & 42,344 & 41,319 & 42,671 & 46,311 & 49,517 & 55,375 \\
\hline Domestic revenue (incl. net lending) & 32,757 & 36,267 & 29,690 & 14,629 & 33,824 & 31,644 & 35,239 & 38,776 & 42,324 & 48,931 \\
\hline Tax revenue & 29,585 & 30,302 & 25,676 & 12,889 & 28,737 & 27,088 & 29,948 & 32,908 & 35,773 & 41,638 \\
\hline Income and profit taxes & 8,525 & 8,900 & 8,059 & 4,143 & 9,067 & 8,152 & 9,162 & 10,067 & 10,944 & 12,590 \\
\hline Personal income tax & 4,824 & 5,365 & 4,452 & 2,270 & 5,268 & 4,593 & 5,323 & 5,849 & 6,358 & 7,315 \\
\hline Corporate income tax & 3,701 & 3,535 & 3,607 & 1,873 & 3,799 & 3,559 & 3,839 & 4,218 & 4,585 & 5,275 \\
\hline Consumption taxes & 13,300 & 14,508 & 11,300 & 5,846 & 12,505 & 12,665 & 13,547 & 14,886 & 16,182 & 18,729 \\
\hline International trade taxes & 6,110 & 5,917 & 5,219 & 2,463 & 5,917 & 5,250 & 5,979 & 6,570 & 7,142 & 8,360 \\
\hline Other taxes & 1,650 & 978 & 1,098 & 437 & 1,248 & 1,021 & 1,261 & 1,385 & 1,506 & 1,959 \\
\hline Nontax revenue & 3,102 & 5,944 & 4,014 & 1,725 & 5,087 & 4,556 & 5,291 & 5,868 & 6,550 & 7,294 \\
\hline External grants & 6,430 & 7,372 & 6,850 & 1,939 & 8,520 & 9,675 & 7,433 & 7,535 & 7,194 & 6,444 \\
\hline Capital grants & 4,790 & 5,478 & 4,670 & 1,573 & 6,201 & 6,697 & 4,380 & 5,636 & 5,405 & 4,444 \\
\hline Budget support (incl. food aid) & 1,640 & 1,894 & 2,180 & 366 & 2,319 & 2,978 & 3,053 & 1,899 & 1,789 & 2,000 \\
\hline Total expenditure & 40,715 & 51,435 & 44,493 & 21,420 & 57,094 & 59,779 & 59,104 & 58,911 & 56,082 & 61,445 \\
\hline Recurrent expenditure & 23,264 & 27,984 & 24,602 & 12,320 & 28,920 & 28,763 & 31,860 & 33,330 & 34,952 & 38,498 \\
\hline Primary recurrent expenditure & 21,367 & 25,692 & 22,705 & 11,389 & 26,408 & 26,232 & 29,367 & 30,849 & 32,326 & 36,318 \\
\hline Wages and salaries & 13,518 & 15,950 & 14,844 & 7,727 & 17,129 & 17,140 & 17,882 & 18,508 & 19,156 & 21,933 \\
\hline Goods and services & 2,048 & 2,964 & 2,341 & 1,043 & 3,162 & 2,891 & 3,214 & 3,362 & 3,450 & 3,796 \\
\hline Transfers and subsidies & 4,781 & 4,924 & 4,874 & 2,340 & 5,173 & 4,982 & 6,036 & 6,253 & 6,464 & 7,006 \\
\hline Transfers & 3,910 & 4,667 & 4,029 & 2,147 & 4,800 & 4,472 & 5,130 & 5,309 & 5,495 & 6,047 \\
\hline Subsidies & 871 & 258 & 845 & 193 & 372 & 510 & 906 & 944 & 969 & 959 \\
\hline Other expenditures & 1,020 & 1,854 & 646 & 279 & 944 & 1,219 & 2,236 & 2,726 & 3,256 & 3,582 \\
\hline Domestic interest payments & 1,348 & 1,570 & 1,305 & 616 & 1,727 & 1,747 & 1,505 & 1,172 & 1,023 & 1,063 \\
\hline External interest payments & 548 & 722 & 592 & 315 & 784 & 784 & 988 & 1,308 & 1,603 & 1,117 \\
\hline Capital expenditure & 16,264 & 20,990 & 17,823 & 8,757 & 27,974 & 31,016 & 27,244 & 25,581 & 21,130 & 22,947 \\
\hline Foreign financed & 11,701 & 13,939 & 12,346 & 6,840 & 22,976 & 26,556 & 22,015 & 20,353 & 15,011 & 14,345 \\
\hline Domestically financed & 4,562 & 7,052 & 5,477 & 1,917 & 4,998 & 4,460 & 5,229 & 5,229 & 6,119 & 8,602 \\
\hline Other expenditures (incl. arrears clearance) & 1,188 & 2,460 & 2,068 & 344 & 200 & 0 & 0 & 0 & 0 & 0 \\
\hline Overall balance, including grants & $-1,529$ & $-7,796$ & $-7,953$ & $-4,852$ & $-14,749$ & $-18,460$ & $-16,433$ & $-12,600$ & $-6,565$ & $-6,070$ \\
\hline Financing & 1,529 & 7,796 & 7,953 & 4,852 & 14,749 & 18,460 & 16,433 & 12,600 & 6,565 & 6,070 \\
\hline Foreign, net & 3,495 & 6,756 & 6,301 & 4,352 & 16,343 & 16,222 & 13,926 & 12,102 & 7,164 & 5,332 \\
\hline Drawings & 5,381 & 8,884 & 8,248 & 5,267 & 18,456 & 18,288 & 15,973 & 14,192 & 9,189 & 7,901 \\
\hline Amortization & $-1,886$ & $-2,127$ & $-1,947$ & -915 & $-2,114$ & $-2,066$ & $-2,047$ & $-2,090$ & $-2,025$ & $-2,569$ \\
\hline Domestic, net & $-1,138$ & 1,039 & 965 & 248 & $-1,731$ & 2,238 & 2,506 & 499 & -600 & 738 \\
\hline Net domestic borrowing & $-1,972$ & 1,759 & 1,900 & 268 & 1,528 & 4,046 & 3,798 & 1,893 & 940 & 0 \\
\hline Banking system & $-2,008$ & $\ldots$ & 518 & $-2,651$ & 2,961 & 4,046 & 3,798 & 1,893 & 940 & 0 \\
\hline Nonbanks & 36 & $\ldots$ & 1,382 & 2,919 & $-1,433$ & 0 & 0 & 0 & 0 & 0 \\
\hline Privatization and other sales of assets & 834 & -720 & -106 & -20 & 229 & -400 & 98 & -20 & -167 & 738 \\
\hline Accounts payable, net (o/w onlending) & 0 & $\ldots$ & -829 & 0 & $-3,489$ & $-1,408$ & $-1,390$ & $-1,374$ & $-1,372$ & 0 \\
\hline Net errors and omissions & -828 & 0 & 687 & 253 & 0 & 0 & 0 & 0 & 0 & 0 \\
\hline Financing gap & 0 & 0 & 0 & 0 & 138 & 0 & 0 & 0 & 0 & 0 \\
\hline \multicolumn{11}{|l|}{ Memorandum items: } \\
\hline Overall balance, including grants ${ }^{2}$ & -955 & $-5,336$ & $-6,278$ & $-4,852$ & $-14,549$ & $-18,460$ & $-16,433$ & $-12,600$ & $-6,565$ & $-6,070$ \\
\hline Arrears clearance & 574 & 2,460 & 1,675 & 0 & 200 & 0 & 0 & 0 & 0 & 0 \\
\hline Net domestic borrowing ${ }^{2}$ & $-2,546$ & -701 & 225 & 268 & 1,328 & 4,046 & 3,798 & 1,893 & 940 & 0 \\
\hline Primary basic balance ${ }^{3}$ & 6,828 & & 1,508 & 979 & 2,218 & 2,418 & 643 & 2,698 & 3,879 & 5,685 \\
\hline Recurrent domestic balance ${ }^{4}$ & 9,493 & 8,283 & 5,088 & 2,309 & 4,904 & 2,881 & 3,379 & 5,446 & 7,372 & 10,433 \\
\hline External flows ${ }^{5}$ & 11,811 & 16,255 & 15,098 & 7,205 & 26,976 & 27,963 & 23,405 & 21,727 & 16,383 & 14,345 \\
\hline External debt service (percent of domestic revenue) & 7.4 & 7.9 & 8.6 & 8.4 & 8.6 & 9.0 & 8.6 & 8.8 & 8.6 & 7.5 \\
\hline Domestic debt 67 & 20,606 & 19,905 & 20,830 & $\ldots$ & 24,676 & 20,830 & 28,474 & 30,367 & 31,306 & 31,306 \\
\hline
\end{tabular}

Sources: Ministry of Finance, Bank of Cape Verde, and IMF staff estimates and projections.

${ }^{1}$ Medium-Term Fiscal Framework

${ }^{2}$ Excluding clearance of arrears and accounts payable

${ }^{3}$ Excluding grants, interest, and foreign-financed expenditure.

${ }^{4}$ Domestic revenue - recurrent expenditure.

${ }^{5}$ External grants + foreign financing.

${ }^{6}$ Including arrears and accounts payable, net of deposits

${ }^{7}$ On the basis of the residency of the creditor or the currency of denomination of the debt, following the "Staff Guidance Note on Debt Limits in FundSupported Programs" (Policy Paper, December 22, 2009) 
Table 3. Cape Verde: Fiscal Operations of the Central Government, 2008-14

\begin{tabular}{|c|c|c|c|c|c|c|c|c|c|c|}
\hline & \multirow[t]{2}{*}{2008} & \multicolumn{2}{|c|}{2009} & \multicolumn{3}{|c|}{2010} & \multirow{2}{*}{$\begin{array}{c}2011 \\
\text { Prog. }\end{array}$} & \multirow{2}{*}{$\frac{2012}{\text { MTFF }^{1}}$} & \multirow{2}{*}{2013} & \multirow{2}{*}{$\begin{array}{l}2014 \\
\text { Proj. }\end{array}$} \\
\hline & & Budget & Prel & $\mathrm{H} 1$ & Budget & Proj. & & & & \\
\hline Revenue, grants, and net lending & 33.6 & 34.6 & 29.0 & 12.1 & 30.9 & 30.1 & 28.6 & 28.3 & 27.4 & 27.8 \\
\hline Domestic revenue (incl. net lending) & 28.1 & 28.8 & 23.6 & 10.7 & 24.7 & 23.1 & 23.6 & 23.7 & 23.4 & 24.6 \\
\hline Tax revenue & 25.3 & 24.1 & 20.4 & 9.4 & 20.9 & 19.7 & 20.1 & 20.1 & 19.8 & 20.9 \\
\hline Income and profit taxes & 7.3 & 7.1 & 6.4 & 3.0 & 6.6 & 5.9 & 6.1 & 6.1 & 6.0 & 6.3 \\
\hline Consumption taxes & 11.4 & 11.5 & 9.0 & 4.3 & 9.1 & 9.2 & 9.1 & 9.1 & 8.9 & 9.4 \\
\hline International trade taxes & 5.2 & 4.7 & 4.1 & 1.8 & 4.3 & 3.8 & 4.0 & 4.0 & 3.9 & 4.2 \\
\hline Other taxes & 1.4 & 0.8 & 0.9 & 0.3 & 0.9 & 0.7 & 0.8 & 0.8 & 0.8 & 1.0 \\
\hline Nontax revenue & 2.7 & 4.7 & 3.2 & 1.3 & 3.7 & 3.3 & 3.5 & 3.6 & 3.6 & 3.7 \\
\hline External grants & 5.5 & 5.9 & 5.4 & 1.4 & 6.2 & 7.1 & 5.0 & 4.6 & 4.0 & 3.2 \\
\hline Capital grants & 4.1 & 4.3 & 3.7 & 1.1 & 4.5 & 4.9 & 2.9 & 3.4 & 3.0 & 2.2 \\
\hline Budget support (incl. food aid) & 1.4 & 1.5 & 1.7 & 0.3 & 1.7 & 2.2 & 2.0 & 1.2 & 1.0 & 1.0 \\
\hline Total expenditure & 34.9 & 40.8 & 35.3 & 15.6 & 41.6 & 43.6 & 39.6 & 36.0 & 31.0 & 30.9 \\
\hline Recurrent expenditure & 19.9 & 22.2 & 19.5 & 9.0 & 21.1 & 21.0 & 21.3 & 20.3 & 19.3 & 19.3 \\
\hline Primary recurrent expenditure & 18.3 & 20.4 & 18.0 & 8.3 & 19.3 & 19.1 & 19.7 & 18.8 & 17.9 & 18.2 \\
\hline Wages and salaries & 11.6 & 12.7 & 11.8 & 5.6 & 12.5 & 12.5 & 12.0 & 11.3 & 10.6 & 11.0 \\
\hline Goods and services & 1.8 & 2.4 & 1.9 & 0.8 & 2.3 & 2.1 & 2.2 & 2.1 & 1.9 & 1.9 \\
\hline Transfers and subsidies & 4.1 & 3.9 & 3.9 & 1.7 & 3.8 & 3.6 & 4.0 & 3.8 & 3.6 & 3.5 \\
\hline Transfers & 3.3 & 3.7 & 3.2 & 1.6 & 3.5 & 3.3 & 3.4 & 3.2 & 3.0 & 3.0 \\
\hline Subsidies & 0.7 & 0.2 & 0.7 & 0.1 & 0.3 & 0.4 & 0.6 & 0.6 & 0.5 & 0.5 \\
\hline Other expenditures & 0.9 & 1.5 & 0.5 & 0.2 & 0.7 & 0.9 & 1.5 & 1.7 & 1.8 & 1.8 \\
\hline Domestic interest payments & 1.2 & 1.2 & 1.0 & 0.4 & 1.3 & 1.3 & 1.0 & 0.7 & 0.6 & 0.5 \\
\hline External interest payments & 0.5 & 0.6 & 0.5 & 0.2 & 0.6 & 0.6 & 0.7 & 0.8 & 0.9 & 0.6 \\
\hline Capital expenditure & 13.9 & 16.7 & 14.1 & 6.4 & 20.4 & 22.6 & 18.3 & 15.6 & 11.7 & 11.5 \\
\hline Foreign financed & 10.0 & 11.1 & 9.8 & 5.0 & 16.7 & 19.4 & 14.8 & 12.4 & 8.3 & 7.2 \\
\hline Domestically financed & 3.9 & 5.6 & 4.3 & 1.4 & 3.6 & 3.3 & 3.5 & 3.2 & 3.4 & 4.3 \\
\hline Other expenditures (incl. arrears clearance) & 1.0 & 2.0 & 1.6 & 0.3 & 0.1 & 0.0 & 0.0 & 0.0 & 0.0 & 0.0 \\
\hline Overall balance, including grants & -1.3 & -6.2 & -6.3 & -3.5 & -10.8 & -13.5 & -11.0 & -7.7 & -3.6 & -3.0 \\
\hline Financing & 1.3 & 6.2 & 6.3 & 3.5 & 10.8 & 13.5 & 11.0 & 7.7 & 3.6 & 3.0 \\
\hline Foreign, net & 3.0 & 5.4 & 5.0 & 3.2 & 11.9 & 11.8 & 9.3 & 7.4 & 4.0 & 2.7 \\
\hline Drawings & 4.6 & 7.1 & 6.5 & 3.8 & 13.5 & 13.3 & 10.7 & 8.7 & 5.1 & 4.0 \\
\hline Balance of payments, budget & 0.7 & 0.9 & 2.0 & 0.0 & 0.5 & 2.2 & 1.1 & 0.7 & 0.2 & 0.1 \\
\hline Project loans & 4.0 & 6.1 & 4.5 & 3.8 & 12.9 & 11.1 & 9.6 & 7.9 & 4.9 & 3.9 \\
\hline Amortization & -1.6 & -1.7 & -1.5 & -0.7 & -1.5 & -1.5 & -1.4 & -1.3 & -1.1 & -1.3 \\
\hline Domestic, net & -1.0 & 0.8 & 0.8 & 0.2 & -1.3 & 1.6 & 1.7 & 0.3 & -0.3 & 0.4 \\
\hline Net domestic borrowing & -1.7 & 1.4 & 1.5 & 0.2 & 1.1 & 2.9 & 2.5 & 1.2 & 0.5 & 0.0 \\
\hline Privatization and other sales of assets & 0.7 & -0.6 & -0.1 & 0.0 & 0.2 & -0.3 & 0.1 & 0.0 & -0.1 & 0.4 \\
\hline Accounts payable, net (o/w onlending) & 0.0 & $\ldots$ & -0.7 & $\ldots$ & -2.5 & -1.0 & -0.9 & -0.8 & -0.8 & 0.0 \\
\hline Net errors and omissions & -0.7 & 0.0 & 0.5 & 0.2 & 0.0 & 0.0 & 0.0 & 0.0 & 0.0 & 0.0 \\
\hline Financing gap & 0.0 & 0.0 & 0.0 & 0.0 & 0.1 & 0.0 & 0.0 & 0.0 & 0.0 & 0.0 \\
\hline \multicolumn{11}{|l|}{ Memorandum items: } \\
\hline Overall balance, including grants ${ }^{2}$ & -0.8 & -4.2 & -5.0 & -3.5 & -10.6 & -13.5 & -11.0 & -7.7 & -3.6 & -3.0 \\
\hline Arrears clearance & 0.5 & 2.0 & 1.3 & 0.0 & 0.1 & 0.0 & 0.0 & 0.0 & 0.0 & 0.0 \\
\hline Net domestic borrowing ${ }^{2}$ & -2.2 & -0.6 & 0.2 & 0.2 & 1.0 & 2.9 & 2.5 & 1.2 & 0.5 & 0.0 \\
\hline Primary basic balance ${ }^{3}$ & 5.8 & $\ldots$ & -4.8 & 0.7 & 1.6 & 1.8 & 0.4 & 1.6 & 2.1 & 2.9 \\
\hline Recurrent domestic balance ${ }^{4}$ & 8.1 & 6.6 & 4.0 & 1.7 & 3.6 & 2.1 & 2.3 & 3.3 & 4.1 & 5.2 \\
\hline External flows ${ }^{5}$ & 10.1 & 12.9 & 10.4 & 5.3 & 19.7 & 20.4 & 15.7 & 13.3 & 9.1 & 7.2 \\
\hline Domestic debt 6,7 & 17.7 & 15.8 & 16.5 & $\ldots$ & 18.0 & 15.2 & 19.1 & 18.5 & 17.3 & 15.7 \\
\hline Nominal GDP (millions of CVEsc) & 116,724 & 125,966 & 125,966 & 137,179 & 137,179 & 137,179 & 149,227 & 163,784 & 180,958 & 199,111 \\
\hline
\end{tabular}

Sources: Ministry of Finance, Bank of Cape Verde, and IMF staff estimates and projections.

${ }^{1}$ Medium-Term Fiscal Framework

${ }^{2}$ Excluding clearance of arrears and accounts payable

${ }^{3}$ Excluding grants, interest, and foreign-financed expenditure.

${ }^{4}$ Domestic revenue - recurrent expenditure.

${ }^{5}$ External grants + foreign financing.

${ }^{6}$ Including arrears and accounts payable, net of deposits

${ }^{6}$ Including arrears and accounts payable, net of deposits

${ }^{7}$ On the basis of the residency of the creditor or the currency of denomination of the debt, following the "Staff Guidance Note on Debt Limits in Fund-Supported Programs" (Policy Paper, December 22, 2009) 
Table 4. Cape Verde: Balance of Payments, 2008-14

(Millions of euros, unless otherwise indicated)

\begin{tabular}{|c|c|c|c|c|c|c|c|}
\hline & 2008 & 2009 & 2010 & 2011 & 2012 & 2013 & 2014 \\
\hline & & \multicolumn{6}{|c|}{ Projections } \\
\hline Current account balance (including official transfers) & -135 & -112 & -226 & -228 & -226 & -204 & -175 \\
\hline Trade balance & -487 & -453 & -555 & -559 & -609 & -635 & -676 \\
\hline Exports, f.o.b. & 78 & 65 & 77 & 87 & 97 & 109 & 122 \\
\hline Imports, f.o.b. & -566 & -518 & -633 & -646 & -706 & -744 & -798 \\
\hline Services (net) & 168 & 130 & 113 & 120 & 179 & 218 & 285 \\
\hline Credit & 406 & 356 & 372 & 418 & 486 & 565 & 668 \\
\hline Of which: tourism & 230 & 193 & 200 & 224 & 263 & 309 & 369 \\
\hline Debit & -238 & -226 & -259 & -297 & -306 & -347 & -383 \\
\hline Income (net) & -32 & -37 & -42 & -44 & -56 & -60 & -65 \\
\hline Credit & 19 & 16 & 15 & 15 & 17 & 18 & 21 \\
\hline Debit & -51 & -53 & -58 & -59 & -72 & -78 & -86 \\
\hline Government interest & -5 & -4 & -4 & -6 & -8 & -9 & -10 \\
\hline Interest by other sectors & -19 & -16 & -22 & -24 & -25 & -26 & -27 \\
\hline Income on direct investment and other income & -27 & -34 & -31 & -29 & -39 & -44 & -49 \\
\hline Current transfers (net) & 217 & 249 & 259 & 255 & 259 & 273 & 281 \\
\hline Government & 71 & 86 & 88 & 67 & 68 & 65 & 58 \\
\hline Others, incl. emigrant remittances & 146 & 163 & 172 & 187 & 190 & 207 & 223 \\
\hline Capital and financial account (net) & 237 & 177 & 249 & 252 & 264 & 239 & 247 \\
\hline Capital transfers & 19 & 20 & 26 & 19 & 30 & 28 & 40 \\
\hline Direct investment (net) & 143 & 86 & 87 & 113 & 130 & 146 & 159 \\
\hline Portfolio investment & 0 & 4 & 1 & 0 & 0 & 0 & 0 \\
\hline Other investments, central government & 29 & 57 & 148 & 127 & 110 & 65 & 48 \\
\hline Net official flows & 29 & 57 & 148 & 127 & 110 & 65 & 48 \\
\hline Disbursements & 46 & 75 & 166 & 145 & 129 & 83 & 72 \\
\hline Amortization & -17 & -18 & -18 & -18 & -18 & -18 & -23 \\
\hline Other investments, non-central government & 46 & 10 & -13 & -7 & -6 & -1 & -1 \\
\hline Commercial banks (net) & 44 & 8 & 0 & 0 & 1 & 1 & 1 \\
\hline Commercial credit (net) & 6 & 4 & 0 & 0 & 0 & 0 & 0 \\
\hline Other & -4 & -2 & -13 & -8 & -7 & -2 & -1 \\
\hline Net errors and omissions & -84 & -77 & 0 & 0 & 0 & 0 & 0 \\
\hline Overall balance & 18 & -12 & 23 & 24 & 38 & 35 & 72 \\
\hline Financing & -18 & 12 & -23 & -24 & -38 & -35 & -72 \\
\hline Gross international reserves ( - accumulation) & -21 & 4 & -23 & -24 & -38 & -35 & -72 \\
\hline Exceptional financing & 3 & 8 & 0 & 0 & 0 & 0 & 0 \\
\hline Financing gap & 0 & 0 & 0 & 0 & 0 & 0 & 0 \\
\hline \multicolumn{8}{|l|}{ Memorandum items: } \\
\hline Current account (including official transfers; percent of GDP) & -12.8 & -9.8 & -18.1 & -16.8 & -15.2 & -12.4 & -9.7 \\
\hline Current account (excluding official transfers; percent of GDP) & -19.5 & -17.3 & -25.2 & -21.8 & -19.8 & -16.4 & -12.9 \\
\hline Overall balance (percent of GDP) & 1.7 & -1.0 & 1.9 & 1.8 & 2.5 & 2.1 & 4.0 \\
\hline Gross international reserves & 280 & 275 & 299 & 323 & 361 & 395 & 467 \\
\hline Months of current year's import of goods and services & 4.2 & 4.4 & 4.0 & 4.1 & 4.3 & 4.3 & 4.7 \\
\hline Months of next year's import of goods and services & 4.5 & 3.7 & 3.8 & 3.8 & 4.0 & 4.0 & 4.3 \\
\hline External public debt & 501 & 541 & 783 & 915 & 1,027 & 1,098 & 1,152 \\
\hline
\end{tabular}

Sources: Bank of Cape Verde; and IMF staff estimates and projections. 
Table 5. Cape Verde: Monetary Survey, 2007-14



Sources: Bank of Cape Verde, and IMF staff estimates and projections. 
Table 6. Cape Verde: Central Bank Survey, 2007-14

\begin{tabular}{|c|c|c|c|c|c|c|c|c|}
\hline & 2007 & 2008 & 2009 & 2010 & 2011 & 2012 & 2013 & 2014 \\
\hline & & & \multicolumn{6}{|c|}{ Projections } \\
\hline & \multicolumn{7}{|c|}{ (Millions of Cape Verde escudos, unless otherw ise specified) } & \\
\hline Net foreign assets & 27,614 & 29,780 & 27,962 & 30,491 & 34,204 & 38,578 & 42,559 & 50,594 \\
\hline Of which: net international reserves & 27,572 & 29,866 & 29,476 & 32,186 & 35,099 & 39,473 & 43,454 & 51,488 \\
\hline Foreign assets & 28,760 & 31,019 & 30,507 & 33,052 & 35,742 & 39,905 & 43,723 & 51,658 \\
\hline Foreign liabilities & $-1,146$ & $-1,239$ & $-2,545$ & $-2,561$ & $-1,538$ & $-1,327$ & $-1,164$ & $-1,064$ \\
\hline Net domestic assets & $-3,574$ & $-3,821$ & $-1,128$ & $-2,417$ & $-3,530$ & $-5,197$ & $-5,880$ & $-10,438$ \\
\hline Net domestic credit & $-9,666$ & $-1,105$ & 1,334 & -65 & $-1,132$ & $-2,750$ & $-3,385$ & $-7,893$ \\
\hline Trust Fund claims & 0 & 4,605 & 4,605 & 4,605 & 4,605 & 4,605 & 4,605 & 4,605 \\
\hline Net claims on central government & $-4,346$ & 79 & $-1,431$ & $-2,431$ & $-4,431$ & $-6,431$ & $-6,931$ & $-8,941$ \\
\hline Credit to central government & 0 & 3,640 & 3,640 & 3,640 & 3,640 & 3,640 & 3,640 & 3,640 \\
\hline Deposits of central government & $-4,346$ & $-3,561$ & $-5,071$ & $-6,071$ & $-8,071$ & $-10,071$ & $-10,571$ & $-12,581$ \\
\hline Credit to the economy & 1,162 & 1,106 & 1,099 & 1,106 & 1,112 & 1,118 & 1,123 & 1,129 \\
\hline Credit to private sector & 1,159 & 1,106 & 1,099 & 1,106 & 1,112 & 1,117 & 1,123 & 1,129 \\
\hline Claims on nonbank financial institutions & 3 & 0 & 0 & 0 & 0 & 0 & 0 & 0 \\
\hline Net Credit to commercial banks & $-6,483$ & $-6,895$ & $-2,939$ & $-3,346$ & $-2,418$ & $-2,042$ & $-2,182$ & $-4,685$ \\
\hline Other items (net) & 6,092 & $-2,715$ & $-2,462$ & $-2,351$ & $-2,398$ & $-2,446$ & $-2,495$ & $-2,545$ \\
\hline Reserve money (M0) & 24,041 & 25,959 & 26,834 & 28,074 & 30,674 & 33,381 & 36,679 & 40,156 \\
\hline Currency issued & 9,983 & 10,350 & 10,635 & 11,228 & 11,910 & 12,840 & 13,812 & 15,139 \\
\hline Deposits of commercial banks & 14,058 & 15,609 & 16,984 & 17,439 & 19,446 & 21,471 & 23,839 & 26,343 \\
\hline \multicolumn{9}{|l|}{ Memorandum items: } \\
\hline Gross international reserves ( $€$ millions) & 259.6 & 280.1 & 275.4 & 298.5 & 322.9 & 360.7 & 395.3 & 467.3 \\
\hline Net international reserves ( $€$ millions) & 250.1 & 270.9 & 267.3 & 291.9 & 318.3 & 358.0 & 394.1 & 467.0 \\
\hline (months of imports) & 3.9 & 4.5 & 3.7 & 3.8 & 3.8 & 4.0 & 4.0 & 4.1 \\
\hline Reserve money (12-month change in percent) & 10.0 & 8.0 & 3.4 & 4.6 & 9.3 & 8.8 & 9.9 & 9.5 \\
\hline
\end{tabular}

Sources: Bank of Cape Verde, and IMF staff estimates and projections. 
Table 7. Cape Verde: Deposit Money Bank Survey, 2007-14

\begin{tabular}{|c|c|c|c|c|c|c|c|c|}
\hline & 2007 & 2008 & 2009 & 2010 & 2011 & 2012 & 2013 & 2014 \\
\hline & & & \multicolumn{6}{|c|}{ Projections } \\
\hline & \multicolumn{7}{|c|}{ (Millions of Cape Verde escudos, unless otherw ise specified) } & \\
\hline Net foreign assets & 3,361 & -789 & -548 & -562 & -617 & -673 & -730 & -788 \\
\hline Foreign assets & 10,001 & 7,134 & 7,107 & 7,132 & 7,230 & 7,331 & 7,434 & 7,539 \\
\hline Foreign liabilities & $-6,640$ & $-7,922$ & $-7,655$ & $-7,693$ & $-7,847$ & $-8,004$ & $-8,164$ & $-8,327$ \\
\hline Of which: nonresident deposits & $-5,198$ & $-5,647$ & $-4,918$ & $-4,942$ & $-5,041$ & $-5,142$ & $-5,245$ & $-5,350$ \\
\hline Net domestic assets & 82,859 & 93,859 & 97,490 & 105,229 & 116,942 & 128,764 & 142,577 & 157,186 \\
\hline Net domestic credit & 93,628 & 107,392 & 113,805 & 128,555 & 144,204 & 158,854 & 177,443 & 196,697 \\
\hline Net claims on general government & 0 & 13,991 & 15,425 & 19,471 & 23,269 & 25,161 & 26,101 & 26,101 \\
\hline Trust Fund claims & 6,433 & 6,433 & 6,433 & 6,433 & 6,433 & 6,433 & 6,433 & 6,433 \\
\hline Other government deposits (INPS) & -514 & -690 & -894 & -903 & -912 & -921 & -930 & -940 \\
\hline Net claims on central government & 10,375 & 7,846 & 8,834 & 12,795 & 16,502 & 18,282 & 19,087 & 18,945 \\
\hline Claims on central government & 13,095 & 10,737 & 11,583 & 15,544 & 19,251 & 21,031 & 21,836 & 21,694 \\
\hline Deposits of central government & $-2,721$ & $-2,891$ & $-2,749$ & $-2,749$ & $-2,749$ & $-2,749$ & $-2,749$ & $-2,749$ \\
\hline Net claims on local government & -310 & 403 & 1,052 & 1,145 & 1,246 & 1,367 & 1,511 & 1,662 \\
\hline Claims on local government & 295 & 772 & 1,409 & 1,534 & 1,669 & 1,832 & 2,024 & 2,227 \\
\hline Deposits of local government & -605 & -369 & -357 & -389 & -423 & -464 & -513 & -565 \\
\hline Credit to the economy & 55,733 & 69,973 & 78,123 & 86,693 & 97,284 & 108,212 & 123,142 & 137,164 \\
\hline Credit to private sector & 54,394 & 69,337 & 77,650 & 86,220 & 96,811 & 107,739 & 122,669 & 136,691 \\
\hline Claims on nonbank financial institutions & 1,337 & 636 & 473 & 473 & 473 & 473 & 473 & 473 \\
\hline Net claims on the Bank of Cape Verde & 21,911 & 23,428 & 20,257 & 22,392 & 23,651 & 25,481 & 28,200 & 33,432 \\
\hline Other items (net) & $-10,770$ & $-13,533$ & $-16,315$ & $-23,326$ & $-27,262$ & $-30,090$ & $-34,866$ & $-39,511$ \\
\hline Deposit liabilities to nonbank residents & 86,220 & 93,070 & 96,942 & 104,667 & 116,325 & 128,091 & 141,847 & 156,398 \\
\hline Local currency deposits & 80,253 & 88,286 & 92,349 & 99,708 & 110,840 & 122,071 & 135,196 & 149,079 \\
\hline Demand deposits & 34,613 & 35,926 & 34,649 & 37,409 & 41,926 & 46,435 & 51,628 & 57,129 \\
\hline Of which: emigrant deposits & 4,285 & 4,972 & 5,413 & 5,844 & 6,549 & 7,254 & 8,065 & 8,924 \\
\hline Time deposits & 45,640 & 52,360 & 57,700 & 62,300 & 68,914 & 75,636 & 83,567 & 91,950 \\
\hline Of which: emigrant deposits & 27,187 & 28,159 & 29,217 & 31,544 & 34,893 & 38,297 & 42,313 & 46,557 \\
\hline Foreign currency deposits & 5,967 & 4,784 & 4,593 & 4,959 & 5,485 & 6,020 & 6,652 & 7,319 \\
\hline Of which: emigrant deposits & 1,081 & 1,225 & 1,369 & 1,478 & 1,634 & 1,794 & 1,982 & 2,181 \\
\hline \multicolumn{9}{|l|}{ Memorandum items: } \\
\hline Emigrant deposits (ratio to total deposits) & 0.38 & 0.37 & 0.37 & 0.37 & 0.37 & 0.37 & 0.37 & 0.37 \\
\hline Other deposits (ratio to broad money) & 0.57 & 0.67 & 0.67 & 0.67 & 0.67 & 0.67 & 0.67 & 0.67 \\
\hline \multicolumn{9}{|l|}{ Composition of emigrant deposits } \\
\hline Local currency & 0.96 & 0.96 & 0.96 & 0.96 & 0.96 & 0.96 & 0.96 & 0.96 \\
\hline Demand & 0.13 & 0.15 & 0.16 & 0.16 & 0.16 & 0.16 & 0.16 & 0.16 \\
\hline Time & 0.83 & 0.85 & 0.84 & 0.84 & 0.84 & 0.84 & 0.84 & 0.84 \\
\hline Foreign currency & 0.04 & 0.04 & 0.04 & 0.04 & 0.04 & 0.04 & 0.04 & 0.04 \\
\hline
\end{tabular}

Sources: Bank of Cape Verde, and IMF staff estimates and projections. 
Table 8. Cape Verde: Financial Soundness of the Banking Sector ${ }^{1}$

(End-year; percent unless otherwise indicated)

\begin{tabular}{|c|c|c|c|c|c|c|c|}
\hline Indicator & 2005 & 2006 & 2007 & 2008 & Jun-09 & Dec-09 & Jun-10 \\
\hline \multicolumn{8}{|l|}{ Capital adequacy } \\
\hline Regulatory capital to risk-weighted assets & 12.1 & 11.1 & 11.4 & 12.0 & 11.3 & 12.0 & 12.0 \\
\hline Regulatory tier 1 capital to risk-weighted assets & 13.4 & 11.3 & 11.8 & 11.5 & 11.3 & 11.8 & 12.0 \\
\hline \multicolumn{8}{|l|}{ Asset quality $^{2}$} \\
\hline Nonperforming loans to total loans & 6.3 & 3.9 & 13.5 & 10.0 & 13.8 & 8.8 & 13.4 \\
\hline Nonperforming loans net of provisions to capital & -2.5 & -1.7 & 91.1 & 42.2 & 76.9 & 25.4 & 62.1 \\
\hline Provisions to nonperforming loans & 104.9 & 104.1 & 35.0 & 53.1 & 41.5 & 65.8 & 46.3 \\
\hline \multicolumn{8}{|l|}{ Earnings and profitability } \\
\hline Return on assets & 0.5 & 1.1 & 1.6 & 1.0 & 0.2 & 0.7 & 0.3 \\
\hline Return on equity & 9.7 & 19.7 & 30.1 & 15.8 & 3.7 & 10.6 & 4.4 \\
\hline Interest margin to gross income & 62.7 & 67.2 & 71.3 & 68.0 & 69.5 & 71.9 & 71.3 \\
\hline Noninterest expenses to gross income & 61.2 & 54.5 & 46.1 & 57.3 & 69.0 & 65.4 & 63.8 \\
\hline \multicolumn{8}{|l|}{ Liquidity $^{3}$} \\
\hline Liquid assets to total assets & 46.2 & 18.3 & 17.7 & 16.6 & 17.2 & 16.4 & 15.6 \\
\hline Liquid assets to short-term liabilities & 170.2 & 46.1 & 42.9 & 43.8 & 46.8 & 49.2 & 48.8 \\
\hline
\end{tabular}

Source: Bank of Cape Verde.

${ }^{1}$ The data since 2008 are based on the IFRS, which are provisional as the BCV is finalizing comparability of data.

2 The NPL ratio for the four onshore banks increased in 2007 because of new stricter loan classification criteria. Thus, the NPLs should be interpreted with cautious since a loan was considered nonperforming if it is only 1 day overdue

${ }^{3}$ Liquid assets include cash in vault and marketable securities. Short-term liabilities include demand deposits.

Table 9. Cape Verde : Proposed Work Program for 2011

\begin{tabular}{lll}
\hline Mission date & Purpose & Board decision \\
\hline September 2010 & New PSI approval & November 22, 2010 \\
May 2011 & First PSI review & end-July 2011 \\
November 2011 & $\begin{array}{l}\text { Second PSI review and possible } \\
\text { negotiation of a new PSI. }\end{array}$ & end-January 2012 \\
\hline
\end{tabular}




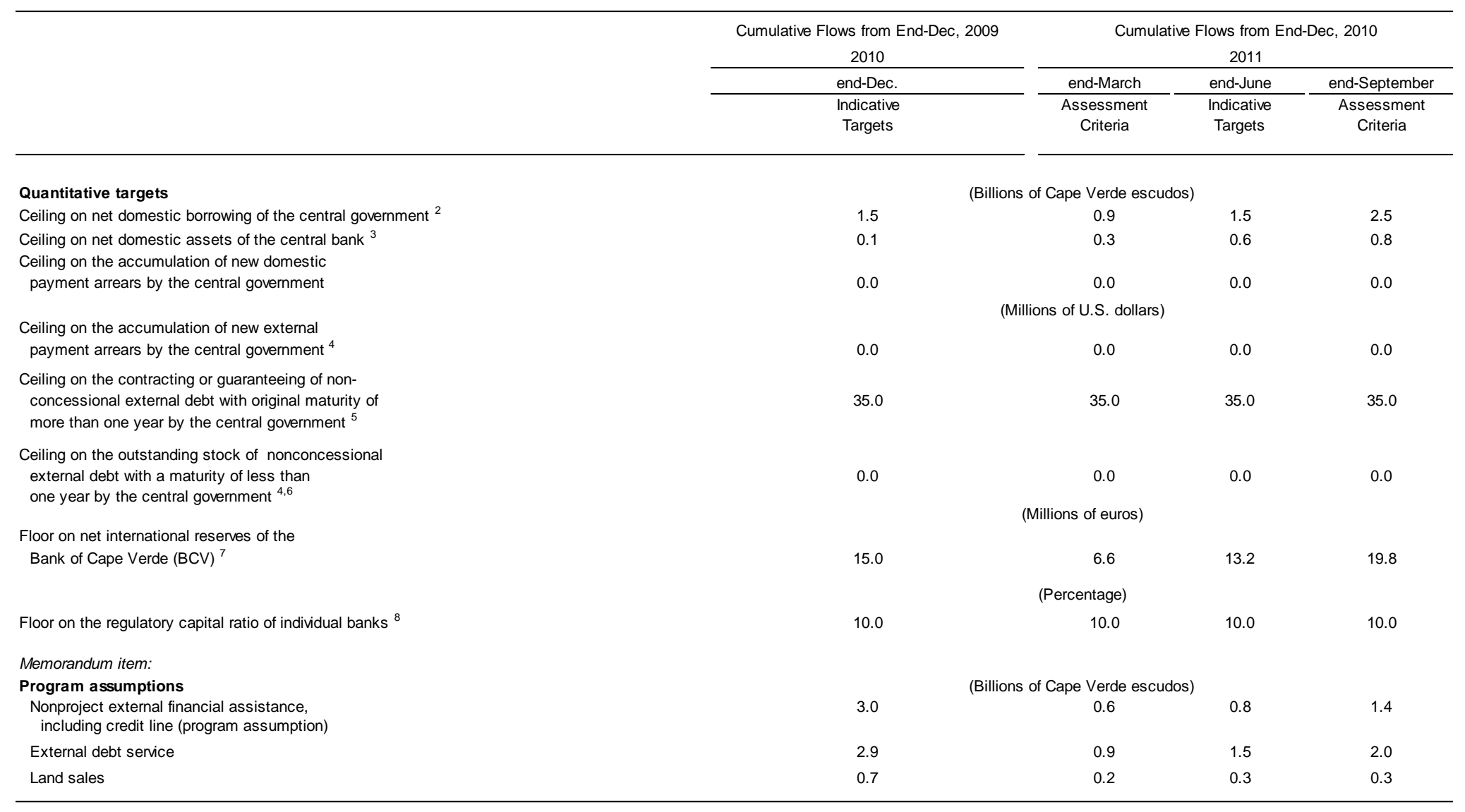

${ }^{1}$ For purposes of calculating program adjusters, foreign currency amounts will be converted at current exchange rates.

${ }^{2}$ Excluding borrowing for clearance of arrears and net late payments. The ceiling will be adjusted upward by the cumulative upward deviation in external debt service, and by the cumulative downward deviation in nonproject external financial assistance and land sales relative to program assumptions. The ceiling will be adjusted downward by the cumulative downward deviation in external debt service and by the in nonproject external financial assistance and land sales relative to program assumptions. The ceiling will be adjusted downward by the cumulative downward deviation in external debt service and by
cumulative upward deviation in land sales relative to program assumptions. The ceiling will be adjusted upward by the costs of restructuring state-owned enterprises up to a limit of CVE 1.5 billion.

${ }^{3}$ The ceiling will be adjusted upward by the cumulative upward deviation in external debt service and by the cumulative downward deviation in external financial assistance, project loans and budget loans relative to program assumptions. The ceiling will be adjusted downward by the cumulative downward deviation in external debt service relative to program assumptions. The ceiling will be adjusted upward by the costs of restructuring state-owned enterprises up to a limit of CVE 1.5 billion.

${ }^{4}$ This assessment criterion is on a continuous basis.

${ }^{5}$ This assessment criterion applies not only to debt as defined in point No. 9 of the Guidelines on Performance Criteria with Respect to Foreign Debt (Decision No. 12274-(00/85), August 24, 2000, as amended on August 31, 2009), but also commitments contracted or guaranteed for which value has not been received. Excluded from this performance criterion are debt rescheduling and debt reorganization, the Portuquese credit line. and borrowinas from the Fund.

${ }^{6}$ The term "debt" has the meaning set forth in point No. 9 of the Guidelines on Performance Criteria with Respect to Foreign Debt (Decision No. 12274-(00/85), August 24, 2000, as amended on August 31, 2009). Excluded from this performance criterion are rescheduling arrangements, the Portuguese credit line, borrowings from the Fund, and import-related financing.

${ }^{7}$ The floor on net international reserves of the Bank of Cape Verde will be adjusted downward by the cumulative upward deviation in external debt senice, by the cumulative downward deviation in external financial assistance, project loans and budget loans relative to program assumptions, and by the costs of restructuring state-owned enterprises up to a limit of 13.6 million euros. The floor will be adjusted upward by the cumulative downward deviation in external debt service relative to program assumptions. Additionaly, in the case Cape Verde participates in any SDR allocation(s) between June 30, 2009 and the test date, the floor on net international reserves will be adjusted upwards by the equivalent of the amount of the cumulative additional SDR allocation(s) up to the test date measured at program exchange

${ }^{8}$ The floor on banks' regulatory capital ratios is an indicative floor for all test dates. This indicative target is continuous. 


\section{APPENDIX I. LETTER OF INTENT}

November 9, 2010

Dominique Strauss-Kahn

Managing Director

International Monetary Fund

$70019^{\text {th }}$ Street, N.W.

Washington, D.C. 20431

USA

Dear Mr. Strauss-Kahn

In July 2010, the government of Cape Verde completed its program supported by the IMF under the Policy Support Instrument (PSI). The PSI provided firm support for our policies for macroeconomic stability, economic growth, and poverty reduction. Looking ahead, the government is seeking to continue its close policy dialogue with the IMF and believes that Cape Verde is well-suited to the PSI: macroeconomic stability has been achieved; financial resources from the Fund are not needed; but the PSI would support the government's ongoing efforts to strengthen policy performance and signal its commitment to sound policies to the international community.

The attached Memorandum of Economic and Financial Policies (MEFP) sets out the government's objectives and policies for 2010-11, for which it requests support under a new 15-month PSI supported program, which will allow the new government to assess the form of its future engagement with the Fund after elections in 2011. Key elements of this program include a prudent management of domestic government debt, a further increase in international reserves, and structural reforms to improve debt management, rationalize tax incentives, enhance monetary operations, and strengthen the financial sector.

The government believes that the policies outlined in the MEFP are adequate to achieve the objectives of the successor PSI-supported program. Under the PSI, the government will regularly update the IMF on economic and policy developments and will provide the data needed for adequate monitoring of the program. During the period of the PSI, Cape Verde will consult with the IMF on adoption of any measures that may be appropriate at the initiative of the government or whenever the Managing Director of the IMF requests such a consultation. We authorize the IMF to publish this letter of intent, the attached MEFP, and the related staff report.

Sincerely yours,

/s/

Cristina Duarte

Minister of Finance 


\section{Attachment I. Memorandum of Economic and Financial Policies}

\section{Recent economic developments}

1. Cape Verde's highly open economy has been quite resilient to the impact of the global recession.

- $\quad$ Real GDP growth slowed in 2009, but remained strong compared to global economic growth. This relative moderation in growth stems from a global slowdown in tourism, FDI flows, and private construction, offsetting fiscal stimulus policies. Leading indicators, such as the confidence barometer, show signs of recovery in 2010; and the growth rate in 2010 should pick up on account of public investment-fueled construction sector, and transportation. The growth rate should then gradually rise in the medium term.

- $\quad$ The fiscal stimulus implemented in 2009, including subsidies, tax cuts, and accelerated investment spending, helped Cape Verde weather the crisis and protect the poor. We used the policy space created by prudent fiscal policies in recent years and additional inexpensive external financing to accelerate the public investment program that was already in place.

- Inflation declined from 6.8 percent (annual average) in 2008 to 1 percent in 2009 and should remain around 3 percent in the medium term.

2. The 2006-10 policy support instrument (PSI) has been very supportive of the Cape Verdean reform agenda and growth policy. The PSI helped consolidate macroeconomic stability, build international reserves buffers, improve public finance management, reduce fiscal risk from state-owned enterprises (SOEs), strengthen the control and supervision of the financial sector, and strengthen the national institutional framework through structural and legislative reforms. The PSI also had a catalytic role in donor support and strengthened the credibility of government policies. During the PSI, Cape Verde joined the World Trade Organization (WTO), graduated from Low Developed Country (LDC) status, and was accepted as a special partner of the European Union (EU). As a facilitator of reforms, the PSI helps to advance the objectives of our Poverty Reduction and Growth Strategy. Cape Verde is on track to achieve most of the Millennium Development Goals (MDGs) by 2015, including halving the 1990 poverty level.

3. Challenges remain for Cape Verde to benefit from the current window of highly concessional financing while ensuring debt sustainability. The rapid implementation of our mostly externally financed investment program is ongoing. During recent years, we have worked to mobilize concessional external financing for our investment program. This work has improved our project implementation ratio from below 60 percent earlier in the decade to around 90 percent currently. The pace of our investment program is expected to continue in 2011-12, but should return to more moderate levels after the completion of current large 
infrastructure projects. The investment program is intended to support long-term private growth based on services exports in line with the Cape Verde Poverty Reduction and Growth Strategy. It addresses critical infrastructure bottlenecks in roads, airports, sea ports, and electricity and water supply. Project appraisals carefully prepared with the help of our development partners confirm that the economic and social return from these projects should be high. The external financing of the investment program, even though on highly concessional terms and on long maturities, has added to the total debt burden, especially the present value (PV) of debt to GDP. Cape Verde's repayment capacity remains strong, the debt service to revenues or export ratios staying well below the thresholds. Cape Verde's strong policies and institutions should help ensure that the growth dividends from the accelerated public investments do materialize and help consolidate debt sustainability in the medium term.

\section{Macroeconomic objectives and policies}

\section{The new 15-month PSI will continue to build foreign reserves and keep the} domestic debt stock low to support the exchange rate peg. Net domestic borrowing will be contained to protect international reserves. In addition, fiscal policy will be orientated toward gradual exit from the current counter-cyclical fiscal measures. As the pace of public investment slows, fiscal deficit is expected to decrease from 13.5 percent of GDP in 2010 to 8 percent in 2012, and decline thereafter. External borrowing will continue, mostly on concessional terms, and will be scaled back to past levels after the completion of the current large infrastructure projects.

\section{Fiscal policy}

5. In 2010-11, fiscal policy will remain anchored on implementing the mediumterm public investment program and minimizing and prudently managing debt accumulation.

- In order to protect international reserves and keep net domestic debt level below 20 percent of GDP, net domestic borrowing in 2011 will continue to be kept low.

- $\quad$ Debt management will be strengthened. A Medium-Term Debt Strategy (MTDS) describing the debt level, composition, and desirable trend will be presented to the Council of Ministers in June 2011. Because the concessional debt window will likely decrease as our income rises, we will increasingly borrow on nonconcessional terms, and the MTDS will help us to prudently manage our debt portfolio. In the medium term, the main objective will be to bring down the PV of debt to GDP.

- $\quad$ To achieve the debt stock alleviation goal, externally financed public investments will have to be scaled back to more modest levels after the current acceleration. Public capital expenditures financed with foreign loans are expected to decline from 
12.3 percent of GDP in 2010 to 4.7 percent in 2013. Moreover, growth dividends from the public investments program should materialize and help achieve the goal of a reduced PV of debt to GDP.

\section{Authorities will continue to improve public financial management and strengthen the tax base.}

- $\quad$ As usual, we will conduct a mid-year fiscal review for 2011, and we will confirm that the external borrowing is consistent with debt sustainability and with the mediumterm debt stock alleviation objective.

- $\quad$ The government has prepared new individual and corporate income tax bills and is revising related legislation to further reduce and streamline the tax incentives and exemptions. The government intends to submit these tax bills to the National Assembly by end-June 2011.

- We will continue to enforce tax collection, especially the 2009-2010 unpaid corporate income taxes and VAT.

7. The government will continue to address contingent liabilities arising from stateowned enterprises (SOEs). To avoid the emergence of fiscal risks, the government has enacted Law of State Enterprises, Public Managers, and Corporate Good Governance. These measures aim to improve the control and monitor of the sector, by establishing a set of rules and procedures which will serve as a framework for the regulation of sector, permitting a re-structuring of the public enterprises in order to become an instrument for economic policies. Moreover, they will allow a change of paradigm in the management of the parastatals, to become result/objective oriented. The electricity company, Electra, and the airline company, TACV, have been put on a restructuring plan to end their structural losses or implicit subsidies. The strategy for Electra - to stabilize costs and reduce reliance on oil imports - is to substitute photovoltaic and wind power (using a public-private partnership) instead of fossil-based generation. After downsizing its staff, TACV improved the quality of its services and renewed its IATA certifications. The TACV presented an improved income statement in 2009 and has recently cleared its arrears to private providers accumulated through 2009. The TACV has signed an agreement with the National Company for Airports and Security (ASA) to repay its arrears and the payment plan is in place. The government is committed to increasing transparency regarding implicit subsidies between SOEs, by making any unpaid claims between SOEs explicit in the contingent liabilities report by June 2011.

\section{In accordance with its poverty reduction strategy, the government is committed} to safeguarding priority spending. Active public policies targeting vulnerable individuals continue to be implemented at a strong pace. Those policies aim at supporting sustainable solutions to unemployment and at fostering the integration of the most needy into the economic and social life of the country. Examples include the reform of the social security system, the intensification of programs to combat poverty, the implementation of the 
National Equality and Gender Equity Plan, and adoption of measures to ensure social reinsertion for children and adolescents in situations of risk. Additionally, the Government is investing heavily in the provision of training that caters to current labor market needs, particularly among the youth. Skills development are also being supported by investments on housing, health, water and sanitation, all of which conducive to a better and more productive life for Cape Verdeans.

\section{Monetary management and financial sector policies}

\section{The Bank of Cape Verde (BCV) will safeguard the exchange rate peg by} continuing to accumulate foreign reserves and enhancing monetary operations. With a largely open capital account, monetary policy will be geared toward stabilizing short-term external capital flows and domestic liquidity. Thus, the BCV lowered its policy rate by 100 basis points to 4.25 percent in January 2010, as foreign reserves built up and the global risk premium narrowed. The BCV will continue to closely monitor capital flows and assess implications for domestic interest rates and credit growth.

\section{We are committed to developing a liquid and dynamic government security} market. We will address gradually market distortions in the primary government security market. We intend to: (i) issue fungible government bonds, Obrigacoes do Tesouro, to reduce market fragmentations and foster liquidity in the secondary market; (ii) use uniform-price auctions in the primary market; (iii) enhance the auction mechanisms by publishing issuance calendars one year in advance to foster a competitive, credible, and transparent public securities market; And (iv) use e-banks, internet, and ATMs to permit individuals to buy the securities in the secondary market.

\section{The government is committed to strengthening the coordination of fiscal and} monetary policy to support the exchange rate peg. Under the new PSI, the Ministry of Finance $(\mathrm{MoF})$ and the BCV will sign a fiscal-monetary coordination agreement. Under the agreement, the BCV will use treasury securities systematically as the main instrument for monetary operations. We will enhance the joint MoF-BCV liquidity committee to assess the implications of fiscal financing needs on the monetary aggregates and international reserves.

12. We will continue to strengthen the financial sector, taking into account some weaknesses identified in the 2008 Financial Sector Assessment Program (FSAP) recommendations.

- $\quad$ The BCV is committed to enhancing the regulatory framework and supervisory capacity. The BCV will ensure sufficient resources to its supervisory body to effectively enhance onsite and offsite inspections. It will continue to strengthen compliance for capital adequacy and update the IMF if any bank falls below regulatory capital requirements. Additionally, though the BCV supervises the insurance sector, in the medium term, it will aim to extend its supervisory perimeter 
to other nonbank financial institutions, including the INPS, according to FSAP recommendations.

- $\quad$ Commercial banks have completed migration and compliance with International Financial Reporting Standards (IFRS) on bank accounting.

- $\quad$ The BCV is working on issuing a financial stability report on a regular basis, starting in the first half of 2011.

- The draft legislation of the new banking law, which will harmonize prudential norms between onshore and offshore banking activities, will be submitted to the National Assembly for approval by end June 2011.

13. We will expand the existing standing financial stability committee at the BCV to include MoF by signing a memorandum of understanding (MoU). The MoU will be signed by minister of finance and the governor of the BCV. It will formalize cooperation arrangements for crisis preparedness and contingency planning and ensure that all members have their own contingency plan and are well-trained. The MoU will: (a) develop a coordinated communication strategy; (b) define common principles for crisis management; (c) describe how agreed procedures are activated in times of crisis; and (d) define channels for information exchange.

\section{Program monitoring}

14. Implementation of the program will be monitored through semiannual reviews conducted by the IMF Executive Board on the basis of quantitative indicators and structural benchmarks. These indicators are defined in the attached TMU. The quantitative indicators for end-March 2011 and end-September 2011 will be assessment criteria, and the quantitative indicators for end-December and end-June will be indicative targets. The program will cover November 2010-November 2011. The first review of the program will be based on the end-March 2011 assessment criteria and is scheduled for completion by endJuly 2011. The second review of the program will be based on the end-September 2011 performance criteria and is scheduled for completion by end- January 2012.

15. To ensure that the program is effectively implemented, the government has set up a PSI monitoring committee. The PSI monitoring committee created for the 2006-10 PSI, under the aegis of Ministry of Finance and BCV - will monitor the implementation of the program for 2010-11. This MEFP will be disseminated within the government, government agencies, public entities, and Cape Verdean society. 
Table 1. Cape Verde: Proposed Structural Benchmarks for 2010-11

\begin{tabular}{|c|c|c|}
\hline $\begin{array}{l}\text { Objective/Macro } \\
\text { Criticality }\end{array}$ & Structural Benchmark & Timing \\
\hline $\begin{array}{l}\text { Strengthen debt } \\
\text { management }\end{array}$ & $\begin{array}{l}\text { Create a medium-term strategy report on the debt level, } \\
\text { composition, and desirable trend and present the report to the } \\
\text { council of ministers }\end{array}$ & end-June 2011 \\
\hline Strengthen tax base & $\begin{array}{l}\text { Submit the draft individual and corporate income tax bills to the } \\
\text { National Assembly }\end{array}$ & end-June 2011 \\
\hline $\begin{array}{l}\text { Promote fiscal } \\
\text { accountability }\end{array}$ & $\begin{array}{l}\text { Present cross debt between SOEs in the contingent liabilities } \\
\text { report and submit at the council of ministers. }\end{array}$ & end-June 2011 \\
\hline $\begin{array}{l}\text { Strengthen the } \\
\text { financial sector }\end{array}$ & $\begin{array}{l}\text { The BCV and Ministry of Finance sign a memorandum of } \\
\text { understanding to expand and formalize the standing financial } \\
\text { stability committee at the BCV }\end{array}$ & end-March 2011 \\
\hline $\begin{array}{l}\text { Strengthen the } \\
\text { financial sector }\end{array}$ & $\begin{array}{l}\text { Submit the new banking law, which unifies the regulatory } \\
\text { framework for onshore and offshore banks, to the National } \\
\text { Assembly }\end{array}$ & end-June 2011 \\
\hline $\begin{array}{l}\text { Strengthen fiscal- } \\
\text { monetary policy } \\
\text { coordination }\end{array}$ & $\begin{array}{l}\text { The BCV and Ministry of Finance sign a fiscal-monetary } \\
\text { coordination agreement }\end{array}$ & $\begin{array}{l}\text { end-December } \\
2010\end{array}$ \\
\hline $\begin{array}{l}\text { Strengthen } \\
\text { securities market }\end{array}$ & $\begin{array}{l}\text { Issue fungible treasury bonds with common face and coupon } \\
\text { values and dates of payments }\end{array}$ & $\begin{array}{l}\text { end-December } \\
2010\end{array}$ \\
\hline
\end{tabular}


Table 2. Cape Verde: Proposed Quantitative Assessment Criteria and Indicative Targets for 2010-11 under the PSI

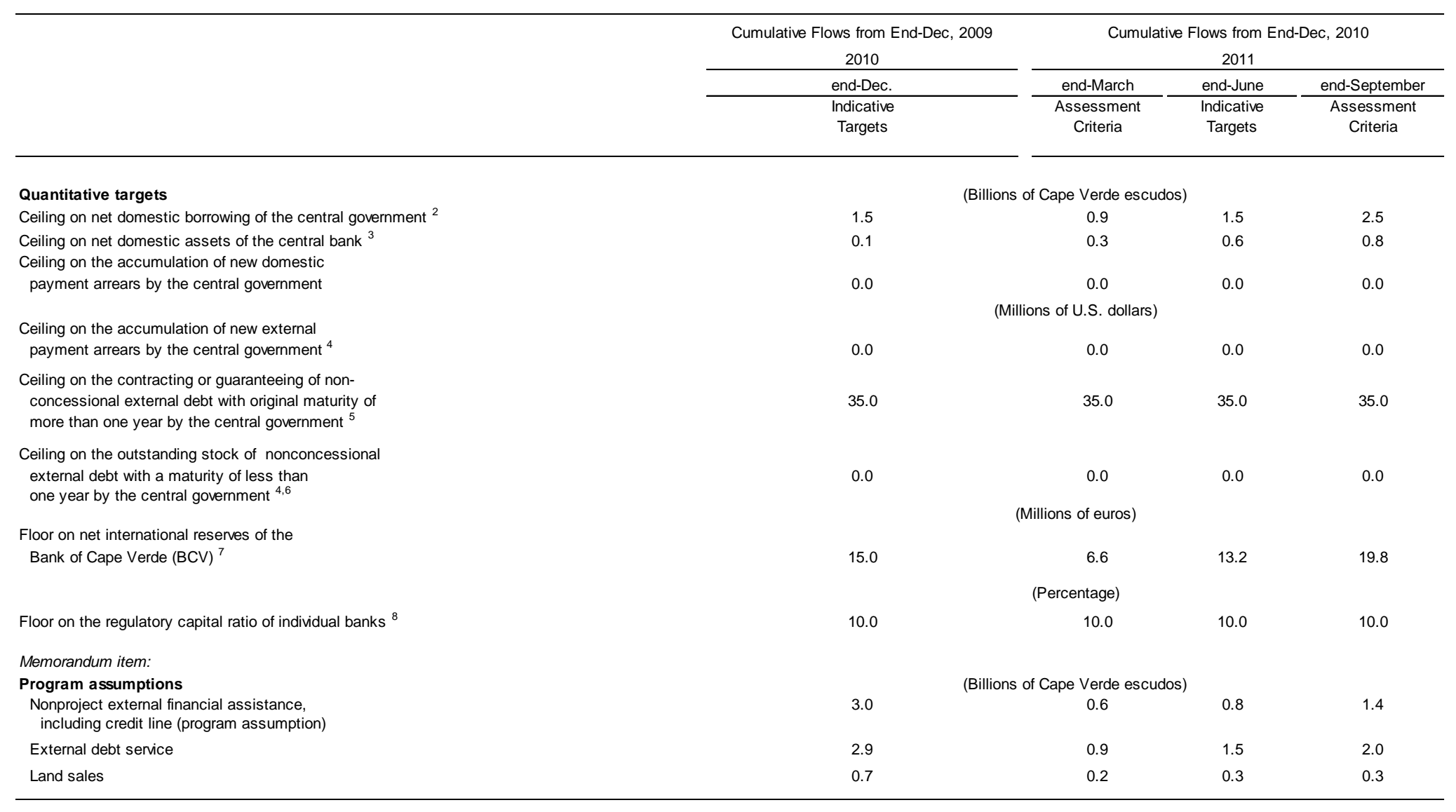

${ }^{1}$ For purposes of calculating program adjusters, foreign currency amounts will be converted at current exchange rates.

2 Excluding borrowing for clearance of arrears and net late payments. The ceiling will be adjusted upward by the cumulative upward deviation in external debt senice, and by the cumulative downward deviation in nonproject external financial assistance and land sales relative to program assumptions. The ceiling will be adjusted downward by the cumulative downward deviation in external debt service and by the cumulative upward deviation in land sales relative to program assumptions. The ceiling will be adjusted upward by the costs of restructuring state-owned enterprises up to a limit of CVE 1.5 billion.

The ceiling will be adjusted upward by the cumulative upward deviation in external debt service and by the cumulative downward deviation in external financial assistance, project loans and budget loans relative to program assumptions. The ceiling will be adjusted downward by the cumulative downward deviation in external debt service relative to program assumptions. The ceiling will be adjusted upward by the costs of restructuring state-owned enterprises up to a limit of CVE 1.5 billion.

${ }^{4}$ This assessment criterion is on a continuous basis.

${ }^{5}$ This assessment criterion applies not only to debt as defined in point No. 9 of the Guidelines on Performance Criteria with Respect to Foreign Debt (Decision No. 12274-(00/85), August 24, 2000, as amended on August 31, 2009), but also commitments contracted or guaranteed for which value has not been received. Excluded from this performance criterion are debt rescheduling and debt reorganization, amended on August 31,2009$)$, but also commitments contronase credit line, and borrowinas from the Fund.

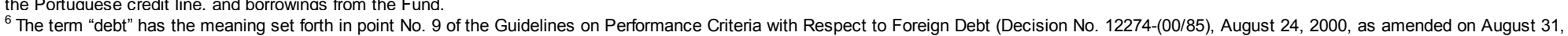
2009). Excluded from this performance criterion are rescheduling arrangements, the Portuguese credit line, borrowings from the Fund, and import-related financing.

${ }^{7}$ The floor on net international reserves of the Bank of Cape Verde will be adjusted downward by the cumulative upward deviation in external debt service, by the cumulative downward deviation in external financial assistance, project loans and budget loans relative to program assumptions, and by the costs of restructuring state-owned enterprises up to a limit of 13.6 million euros. The floor will be adjusted upward by the cumulative downward deviation in external debt service relative to program assumptions. Additionaly, in the case Cape Verde participates in any SDR allocation(s) between June 30, 2009 and the test date, the floor on net international reserves will be adjusted upwards by the equivalent of the amount of the cumulative additional SDR allocation(s) up to the test date measured at program exchange

${ }^{8}$ The floor on banks' regulatory capital ratios is an indicative floor for all test dates. This indicative target is continuous. 


\section{AtTachment II. Technical Memorandum of Understanding}

1. This memorandum sets out the understandings between the Cape Verdean authorities and the IMF staff regarding the definition of assessment criteria and indicative targets and reporting requirements for the seventh and eighth reviews under the Policy Support Instrument.

\section{Quantitative AsSessment CRITERIA AND IndiCATIVE TARgets}

\section{A. Net Domestic Borrowing Excluding for Clearance of Arrears and Net Late Payments}

2. The central government includes all units of budgetary central government. It does not include local government (municipalities) and public corporations

3. Net domestic borrowing excluding for clearance of arrears and net late payments is defined as the cumulative change since the start of the calendar year of the net credit to the central government from the banking and nonbanking sectors less (1) the cumulative clearance during the calendar year of the stock of arrears as of the end of the previous year and (2) the cumulative payments during the first three months of the calendar year of expenses authorized by the previous year's budget, plus the expenses accrued during the current year that will be paid during the first three months of the next calendar year as provisioned for in the budget law (late payments or atrasados).

- $\quad$ The ceiling will be adjusted upward by

$>$ the cumulative upward deviations in external debt service, by

$>$ the cumulative downward deviations in nonproject external financial assistance and land sales relative to program assumptions, and by the costs of restructuring stateowned enterprises up to a limit of CVE 1.5 billion.

- $\quad$ The ceiling will be adjusted downward by

$>$ the cumulative downward deviation in external debt service and by

$>$ the cumulative upward deviation in land sales relative to program assumptions.

4. Net credit to the central government from the banking and nonbanking system is defined as the overall position of the main central government institutions vis-à-vis the banking and nonbanking system - that is, the stock of all outstanding claims on the central government (loans, advances), and all other government debt instruments, such as long-term government securities, held by the BCV, commercial banks, and nonbank institutions, less all deposits held by the central government with the BCV and with commercial banks. The INPS is not included in central government accounts. Net credit to the central government excludes claims on the Trust Fund (TCMFs).

5. Reporting requirements. Data on the implementation of the budget compiled by the Ministry of Finance and Public Administration will be provided on a quarterly basis, to be 
submitted no later than five weeks after the end of each quarter, including (i) government domestic revenue by category; (ii) external budget support grants; (iii) government expenditure, including primary current expenditure, domestic and external interest payments, and capital expenditure, including domestically and budget support financed capital expenditure and estimates of externally project financed capital expenditure; (iv) the gross payment and gross accumulation of domestic accounts payable (atrasados); (v) the gross payment and gross accumulation of domestic payments arrears; (vi) external loan receipts and principal payments; (vii) external arrears payments and accumulation; (viii) bank and nonbank financing; (ix) privatization and land sale receipts; and (x) any other revenue, expenditure, or financing not included above.

6. For the purposes of this memorandum, privatization and land proceeds will be understood to mean all monies received by the government from the sale or concessioning of a public company, organization, or facility to a private company or companies, organization(s), or individual(s), as well as any proceeds generated from the sale of government land and the liquidation of a public company, less restructuring costs.

\section{B. Net Domestic Assets of the Central Bank}

7. The ceiling on the cumulative change, from the beginning of calendar-year 2009, in net domestic assets of the BCV constitutes an assessment criterion. Net domestic assets (NDA) of the $\mathbf{B C V}$ are defined as reserve money minus net foreign assets of the $\mathrm{BCV}$, evaluated at the current end-of-period exchange rates.

- $\quad$ The program ceilings for NDA will be adjusted upward by

$>\quad$ the cumulative upward deviations in external debt service, by

$>\quad$ the cumulative downward deviations in external financial assistance, and project and budget loans relative to program assumptions, and by the costs of restructuring stateowned enterprises up to a limit of CVE 1.5 billion.

- $\quad$ The ceiling will be adjusted downward by the cumulative downward deviation in external debt service relative to program assumptions.

For purposes of calculating the adjusters, these flows will be valued at current exchange rates. Reserve money comprises bank reserves and deposits of the monetary institutions and private sector with the central bank, as well as cash in circulation.

8. Reporting requirements. The preliminary monthly balance sheets of the BCV and the consolidated commercial banks will be transmitted on a monthly basis, with a maximum delay of five weeks. The definitive version of the monthly balance sheet of the BCV will be provided as soon as available. 


\section{Ceiling on Nonconcessional External Debt Contracted or Guaranteed by the Central Government}

9. External public debt (long-term, medium-term, and short-term) is defined as debt to nonresidents contracted or guaranteed by the central government. ${ }^{1}$ The external public debt comprises the external debt of the central government, the Bank of Cape Verde, and the external debt of the official sector entities and state owned enterprises (SOEs) guaranteed by the central government. External public debt also includes the private external debt to nonresidents for which official guarantees have been extended.

10. Under the program, ceilings on medium- and long-term, as well as on short-term, nonconcessional external debt constitute assessment criteria. The ceilings on nonconcessional external debt are on a continuous basis. Nonconcessional external debt is defined as debt contracted or guaranteed by the central government with a grant element of less than 35 percent, calculated using currency-specific commercial interest reference rates (CIRRs) published by the Development Assistance Committee of the Organization for Economic Cooperation and Development (OECD). Debt rescheduling and debt reorganization are excluded from the limits on nonconcessional external debt. The assessment criteria on new nonconcessional external debt contracted or guaranteed by the central government (excluding borrowing from the Fund) are specified in Table 1 of the Letter of Intent. Nonconcessional external debt excludes normal short-term (less than one year) import-related financing. The Portuguese government's precautionary credit line (the "Portuguese credit line") in support of the exchange rate peg is also excluded from the definition of nonconcessional external debt. The assessment criteria on external indebtedness applies not only to debt as defined in point No. 9 of the Guidelines on Performance Criteria with Respect to Foreign Debt (Decision No. 12274-(00/85), 8/24/00, as amended August 31, 2009) but also to commitments contracted or guaranteed for which value has not been received.

11. Reporting requirements. The government of Cape Verde will consult with Fund staff before assuming any liabilities in circumstances where they are uncertain whether the instrument in question falls under the assessment criterion. Details of all new external debt (including government guarantees), indicating terms of debt and creditors, will be provided on a quarterly basis within five weeks of the end of each quarter.

\section{Net International Reserves of the Central Bank}

12. The floor on the cumulative change, from the beginning of calendar-year 2009, in net international reserves (NIR) of the BCV constitutes an assessment criterion under the program. The NIR of the BCV are defined as gross international reserves of the BCV net of its external reserve liabilities, calculated at the current exchange rates. Gross reserves of the BCV are

\footnotetext{
${ }^{1}$ At the time of the first PSI review, the definition of external public debt will be broadened to include the five public enterprises currently covered by the government's contingent liabilities report, and the ceiling on nonconcessional external debt will likewise be increased to account for the broader coverage.
} 
those that are readily available (i.e., liquid and marketable and free of any pledges or encumbrances), controlled by the BCV and held for the purposes of meeting balance of payments needs and intervening in foreign exchange markets. They include gold, holdings of SDRs, the reserve position at the IMF, holdings of foreign exchange and traveler's checks, demand and short-term deposits at foreign banks abroad, fixed-term deposits abroad that can be liquidated without penalty, and any holdings of investment-grade securities. External liabilities of the BCV comprise liabilities to nonresidents contracted by the BCV with an original maturity of less than a year, any net off-balance-sheet position of the BCV (futures, forwards, swaps, or options) with either residents and nonresidents, any arrears on principal and interest to external creditors and suppliers, and purchases from the IMF.

- $\quad$ The program floors for the NIR will be adjusted downward by

$>\quad$ the cumulative upward deviations in external debt service, by

$>\quad$ the cumulative downward deviations in external financial assistance, and project and budget loans relative to program assumptions, and by the costs of restructuring state owned enterprises up to a limit of $€ 13.6$ million. For purposes of calculating the adjusters, these flows will be valued at current exchange rates.

- $\quad$ The floor will be adjusted upward by the cumulative downward deviation in external debt service relative to program assumptions. Additionally, in the case Cape Verde participates in any SDR allocation(s) between June 30, 2009 and the test date, this floor will be adjusted upwards by the equivalent of the amount of the cumulative additional SDR allocation(s) up to the test date measured at program exchange rates.

13. Reporting requirements. A table on the NIR prepared by the BCV will be transmitted on weekly basis, with a maximum delay of two weeks.

\section{E. Nonaccumulation of New Domestic Payments Arrears}

14. As part of the program, the government will not accumulate any new domestic payments arrears. This will be monitored through the monthly execution of the cash-flow plan and the corresponding release of budget appropriations. For programming purposes, a domestic payment obligation to suppliers is deemed to be in arrears if it has not been paid within the normal grace period of 60 days (30 days for government salaries and debt service) or such other period either specified by the budget law or contractually agreed with the supplier after the verified delivery of the concerned goods and services, unless the amount or the timing of the payment is subject to good faith negotiations between the government and the creditor.

15. Reporting requirements. The Ministry of Finance and Public Administration, through the D.G.T., will submit on a quarterly basis a detailed table of the stock of domestic payments arrears, including the accumulation, payment, rescheduling and write-off of domestic payments arrears during the quarter. The data are to be provided within four weeks after the end of the quarter. 


\section{F. Nonaccumulation of External Payments Arrears}

16. As part of the program, the government will not accumulate any new external payments arrears on a continuous basis. This will be monitored through the monthly execution of the cash-flow plan and the corresponding release of budget appropriations.

17. External arrears are defined as total external debt-service obligations of the government that have not been paid by the time they are due, except where agreements between the government and creditors explicitly provide for a grace period after such obligations falling due. External arrears exclude arrears on external debt, pending the conclusion of debtrescheduling agreements.

18. Reporting requirements. Data on (i) debt-service payments; and (ii) external arrears accumulation and payments will be transmitted on a quarterly basis by the Ministry of Finance and Public Administration, within five weeks of the end of each quarter. In addition, the government will inform the Fund staff immediately of any accumulation of external arrears.

\section{G. Regulatory Capital Ratio}

19. The floor is defined as the minimum ratio of regulatory capital to risk-weighted assets following the IMF Financial Soundness Indicators Compilation Guide. This will be monitored through the monthly regulatory capital to risk-weighted assets for each individual bank in the financial soundness indicators of the banking sector. The regulatory capital ratio of individual banks will be provided to the IMF on a monthly basis within six weeks after the end of each month.

\section{OTHER DATA REQUiREMENTS}

20. Data on exports and imports, including volume and prices and compiled by the Director of Customs and the BCV, will be transmitted on a quarterly basis within five weeks after the end of each quarter. A preliminary quarterly balance of payments, compiled by the $\mathrm{BCV}$, will be forwarded within five weeks after the end of each quarter.

21. The Statement of Other Economic Flows as defined in the IMF Manual GFSM2001 relative to holding gains/losses of the previous year with Enapor, Electra, ASA, TACV, and IFH will be transmitted on an annual basis within three months after the end of the following year (15 months after the closing date).

22. The consolidated balance sheet of Electra, Enapor, ASA, TACV and IFH relative to the previous year will be transmitted on an annual basis within three months after the end of the following year (15 months after the closing date).

23. The table of Financial Soundness Indicators will be transmitted to the IMF on a monthly basis within six weeks after the end of each month. The nomination of individual banks is optional. 


\section{Appendix II. Debt Sustainability Analysis \\ Prepared by the Staff of the International Monetary Fund \\ In consultation with the World Bank Staff}

November 9, 2010

The debt sustainability outlook for Cape Verde is broadly unchanged compared to the 2009 DSA, but debt levels have increased significantly from the 2008 DSA. The temporary acceleration of external borrowing for investment to address growth and export impeding infrastructure bottlenecks increased debt trajectories relative to projections in the 2008 DSA. As a result, the PV of external debt-to-GDP ratio approaches the risk threshold in 2012 under the baseline and breaches it in some stress tests. However, most of the debt is highly concessional and on terms longer than 10 years, maintaining the debt service ratios far below their thresholds. To restore buffers against future shocks, Cape Verde will need to (i) reduce external borrowing from 2012 onwards, (ii) mobilize domestic revenue, and (iii) keep domestic borrowing low to accumulate foreign reserves and reinforce the credibility of the currency peg.



Source: IMF staff projections. Baseline

\section{BACKGROUND}

1. This report updates the debt sustainability analysis (DSA) prepared in 2009 (IMF Country Report No. 09/328). The baseline scenario reflects the latest IMF discussions with the authorities (September 2010). Since the 2008 DSA, Cape Verde has been adversely affected by the global crisis through lower exports (mainly services) and FDI. These developments reduced GDP growth and contributed to a weakening of the balance of payments and the fiscal accounts. However, economic growth shows clear signs of a recovery in 2010 and is expected to strengthen over the medium term. 
2. The DSA is based on end-2009 data provided by Cape Verde's authorities. The debt data currently covers central government external debt, publically-guaranteed debt, and domestic debt.

3. Public debt is mostly external and highly concessional, and the recent increase in the stock is driven by both multilateral and bilateral creditors. The World Bank Group and the African Development Banks are the major external creditors (more than 60 percent of the total outstanding debt). The debt is mainly denominated in euros and SDRs.

Text Table 1. Cape Verde: Stock of Public Debt, 2008-2009

\begin{tabular}{lrrrrrrrr}
\hline & \multicolumn{2}{c}{ Million of US \$ } & & \multicolumn{2}{c}{ Percent of total } & & \multicolumn{2}{c}{ Percent of GDP } \\
\cline { 2 - 3 } \cline { 8 - 9 } & 2008 & 2009 & & 2008 & 2009 & & 2008 & 2009 \\
\hline Total & 1006.2 & 1154.0 & 100 & 100 & 65.0 & 63.9 \\
External & 671.9 & 774.6 & 66.8 & 67.1 & 47.3 & 47.3 \\
Domestic & 334.3 & 379.3 & 33.2 & 32.9 & 17.7 & 16.5 \\
\hline
\end{tabular}

Source: Cape Verde's authorities; and IMF and World Bank staff estimates.

Cape Verde: Stock of Debt by Currency, 2009



Cape Verde: Composition of Public External Debt, 2009

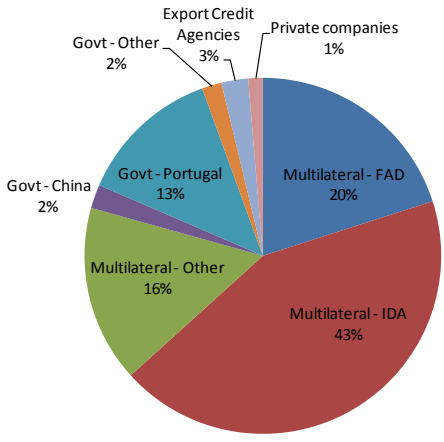

4. The 2009 increase in external debt in percent of GDP ends a five-year decline, from 63 percent in 2003. The substantial rise in 2009 is due to a higher fiscal deficit, but also to the planned acceleration of foreign-financed public investment, in response to the international crisis. However, the 2009 outturn for in public investment was slightly lower than scheduled in the 2008 DSA, as some projects were delayed. Nominal external debt at the end of 2008 and 2009 was 47.3 percent of GDP, with an average maturity of 25 years. A downward revision of historical nominal GDP numbers has mechanically increased the debt ratio in percent of GDP. The appreciation of the dollar in 2009 was unfavorable to Cape Verde, given its peg to the euro, increasing the ratio of foreign debt to GDP. However, authorities have recently canceled or postponed the implementation of a few non-priority 
projects, in order to curb debt accumulation. The share of commercial external debt is only 1 percent.

5. Public debt data does not include a share of the debt of state owned enterprises (SOEs). SOE debt includes loans contracted through the government and already taken into account in the DSA framework. However, SOEs also have arrears to providers and credit lines from banks that are included in contingent liabilities in the risks to the debt outlook. The authorities' report on contingent liabilities to the Council of Ministers in August 2009 estimates the total liabilities owed by the airline, TACV, and the water and electricity company, Electra (both SOEs) at 13 percent of GDP (although the estimated amount needed to recapitalize these firms would be lower, at around 5 percent of GDP).

6. Domestic debt is kept low, in order to protect the peg to the euro. Domestic debt represents one third of total debt and is composed mostly of long-term bonds owed to domestic banks and the public pension fund. It has declined significantly since 2005 as a result of the strong fiscal consolidation under the PSI, which is anchored on keeping domestic borrowing low to build external reserves.

\section{THE DSA BASELINE SCENARIO}

7. Relative to the previous DSA, the macroeconomic framework incorporates the impact of the crisis and the policy response, but remains broadly unchanged regarding the medium and long-term perspectives. More specifically, real GDP growth is

Text Table 2. Cape Verde : Key Macroeconomic Assumptions, 2010-30

expected to rise over the medium term and catch-up to its pre-crisis average, reflecting increased private investment, structural reform and the world recovery. However, the aftermath of the international financial crisis has led to a slight downward revision of real growth projections in the short term. After the global crisis abates, external demand and FDI should recover, supported by the new infrastructure. Growth will be driven by tourism, FDI, and to a lesser extent, remittances. Under the baseline, the growth rate is assumed to recover slowly. The current account balance has been revised up, reflecting weaker domestic demand in the short term and a higher export capacity in the medium term, as a consequence of the accelerated public investment program.

\begin{tabular}{|c|c|c|}
\hline & $2010-11$ & $2012-30$ \\
\hline \multicolumn{3}{|c|}{ Real GDP growth (percent) } \\
\hline Updated & 5.7 & 5.3 \\
\hline Previous ${ }^{1}$ & 5.9 & 5.3 \\
\hline \multicolumn{3}{|c|}{ Fiscal Revenue (percent of GDP) ${ }^{2}$} \\
\hline Updated & 23.3 & 25.3 \\
\hline Previous $^{1}$ & 23.9 & 25.2 \\
\hline \multicolumn{3}{|c|}{ External Current Account (percent of GDP) } \\
\hline Updated & -17.5 & -7.5 \\
\hline Previous $^{1}$ & -22.6 & -7.9 \\
\hline
\end{tabular}

Sources : Cape Verde's authorities; and IMF and world Bank staff estimates.

${ }^{1}$ Previous DSA covers the period 2009-29

${ }^{2}$ Total revenues excluding grants 


\section{Box 1. Macroeconomic Assumptions for the Baseline Scenario}

Real GDP growth slowed due to the global crisis, but is expected to recover over the medium term. Despite the acceleration of infrastructure spending and the lower tax rates, the slowdown in FDI could keep growth below its historical average for some time. The acceleration of public infrastructure spending is unlikely to boost contemporaneous growth because of the small fiscal multipliers caused by high import leakages and because it is not fully offsetting the demand effect of the decline in private investment. While the global shock worsened the near-term growth outlook, Cape Verde's mediumterm growth prospects remain strong.

The acceleration of the public investment program decided in 2009 as a policy response to the crisis is reflected in the short-term growth rate of the construction sector. However, the second order impact of public investment, such as higher competitiveness or positive externalities in the private sector, are not taken into account in the baseline medium and long term growth assumptions.

Inflation is assumed to remain subdued. Given the high import component of domestic demand and the peg to the euro, GDP deflator and consumer price inflation are assumed to be low in the medium and long term.

Current account balance: Imports are assumed to remain broadly flat as a share of GDP. Imports of intermediate goods for tourism are assumed to grow, but this will be largely offset by a decline in other imports. The deceleration of FDI is holding back imports in the near term, and completion of the current large investment projects will contain imports of capital goods in the long run: construction material, equipment, and labor are mostly imported. In addition, as Cape Verde gets wealthier, emigration will slow and the share of remittances and other current transfers in GDP should slowly decline.

Government balances: After a temporary shortfall due to lower activity and tax rate reductions as a policy response to the crisis, tax revenues are assumed to gradually increase and stabilize at around 24 percent of GDP. As the country climbs the income ladder, the informal sector should shrink and the individual and corporate income tax are assumed to increase slightly to reach 8 percent of GDP. At the same time, as a consequence of Economic Partnership Agreements and other Free Trade Agreements (FTA), international trade taxes are decreasing to 4.5 percent of GDP, from 5.5 percent before the crisis. Grants are assumed to gradually decline to 1 percent of GDP. After a peak above 40 percent in the short term on account of accelerated public investment, total expenditures should stabilize around 33 percent, with a wage bill at 12 percent and capital spending at 11 percent. The overall balance (including grants) should fall from a deficit of 15 percent of GDP in 2010 and stabilize at a 2 percent deficit in the medium term. The primary basic balance (excluding grants, interest, and foreign-financed expenditure) is expected to be slightly positive (on average) in the long run.

External assistance: Cape Verde will progressively borrow on less concessional terms. The baseline scenario assumes that the concessionality of new loans will decline in the medium term. With Cape Verde's graduation from least-developed-country status in 2008, it acquired "blend" status with multilateral development agencies, notably the EIB, ADB, and the IBRD. It is now eligible to borrow from their non-concessional windows, and, after a transition period, access to concessional funds will shrink.

Domestic borrowing: The baseline assumes that the authorities will continue to keep domestic borrowing low to support accumulation of foreign reserves and protect the exchange rate peg. Fiscal policy is geared toward accumulation of reserves to 4.5 months of current-year imports in the medium term, and stabilization thereafter. 
The assumption is maintained that fiscal revenue (including grants) will remain relatively flat in the medium and long term, with a substantial shortfall in the short term, reflecting weaker activity and tax cuts in response to the global economic turmoil. Tax revenues are expected to increase slightly in the long run, and will balance the probable decline in external grants. New public borrowing (both domestic and external) is assumed to be moderate over the medium term, reflecting the progressive closing of the concessional external financing window, as well as the commitment to protect the stock of reserves.

\section{EXTERnal DebT SuStainabiLity}

\section{Baseline scenario}

\section{The LIC debt sustainability framework is guided by country-specific debt} burden thresholds for external debt, based on the strength of a country's policies and institutions. Cape Verde now ranks as a 'top performer' under the joint IMF/World Bank debt sustainability framework, based on its three-year moving average CPIA score. The indicative external debt burden thresholds for countries in this category are a present value (PV) of debt-to-exports ratio of 200 percent, a PV of debt-to-revenue ratio of 300 percent, a PV of debt-to-GDP ratio of 50 percent, and debt-service-to-exports and revenues ratios of 25 and 35 percent, respectively.

\section{Cape Verde's} external debt appears sustainable. Under the baseline scenario, all debt indicators remain below their thresholds through 2030 (Text Table 3). The pace of external borrowing will slow sharply from 2012, putting the PV of the external debt-to-GDP ratio on a declining path thereafter. This policy commitment is embedded
Text Table 3. Cape Verde: Baseline Debt Ratios, 2010-30

\begin{tabular}{|c|c|c|c|c|}
\hline & \multirow[t]{2}{*}{ Threshold } & \multirow[b]{2}{*}{2010} & \multirow{2}{*}{$\begin{array}{c}\text { Medium } \\
\text { Term } \\
2010-15\end{array}$} & \multirow{2}{*}{$\begin{array}{c}\begin{array}{c}\text { Long } \\
\text { Term }\end{array} \\
2016-30\end{array}$} \\
\hline & & & & \\
\hline \multicolumn{5}{|l|}{ External } \\
\hline PV of debt-to-GDP & 50.0 & 43.7 & 46.9 & 40.5 \\
\hline PV of debt-to-exports & 200.0 & 121.2 & 119.1 & 73.8 \\
\hline PV of debt-to-revenue & 300.0 & 189.6 & 198.1 & 152.7 \\
\hline Debt service-to-exports & 25.0 & 5.0 & 4.5 & 5.7 \\
\hline Debt service-to-revenue & 35.0 & 7.8 & 7.5 & 12.0 \\
\hline \multicolumn{5}{|l|}{ Fiscal } \\
\hline PV of debt-to-GDP & & 61.7 & 64.6 & 50.9 \\
\hline PV of debt-to-revenue & & 204.9 & 227.5 & 178.4 \\
\hline Debt service-to-revenue & & 17.9 & 19.9 & 27.3 \\
\hline
\end{tabular}

Sources : Cape Verde's authorities; and IMF and world Bank staff estimates. in the MTFF (Medium Term Fiscal Framework) submitted to the National Assembly with the 2010 budget proposal, and in the revised MTFF submitted to staff in September 2010. While the debt-to-GDP ratio will nearly reach the debt burden threshold in 2012, all other debt ratios will remain well below their thresholds, including the debt service ratios. Furthermore, Cape Verde benefits from a large and steady flow of remittances that can increase the repayment capacity of the country. Remittances have averaged 12 percent of GDP over the past ten years and the projections conservatively assume an average of 8 percent of GDP over the next 10 years. 


\section{Alternative scenario and stress tests}

10. Alternative scenarios and bound tests show that all debt indicators except the PV of debt-to-GDP remain well below their thresholds through 2030 . The breach of the PV of debt-to-GDP threshold under the growth shock (B1) is minor (the deviation only amounts to 1 percent of GDP) and lasts for only two years. Other shocks have a more sustained impact. For instance, the export shock (B2) and US deflation shock (B3) result in breaches of 6 percent of GDP in 2012 and last several years. Under the exchange rate shock (B6), which assumes a 30 percent devaluation of the escudo, the PV of the debt-to-GDP ratio breaches the threshold during several years.

11. The other debt ratios provide assurances that Cape Verde's repayment capacity remains strong. Given the high export-to-GDP ratio (over 40 percent) and the strong capacity to collect taxes (the tax collection rate is far above regional standards), the debt-toexport and debt-to-revenue ratios remain well below the thresholds even under extreme scenarios. Moreover, all of Cape Verde's external debt has maturities that are typically longer than 10 years, which alleviates cash flow pressures and limits rollover difficulties. This lessens concerns about the country's ability to repay its debt.

12. A high-growth and a low-growth scenario were constructed to test the sensitivity of debt ratios to growth assumptions. Each scenario assumes that growth will deviate from the baseline by $+/-1.0$ percentage points in 2011-16. As in the baseline, the lowgrowth scenario implies that nominal GDP will be permanently below its pre-crisis trend (the 2011-16 deviations in growth rate are permanent shocks to the GDP level). Because the high-growth scenario assumes growth slightly above its pre-crisis average, GDP will eventually surpass the trend.

13. During the low-growth scenario, the debt service dynamics remain sustainable. The PV of external debt-to-GDP remains below its threshold, with a peak in 2012. The ratios decline slowly thereafter, ending at 36 percent of GDP in 2029, compared with 30 percent of GDP under the baseline scenario. Under the low-growth scenario, the borrowing space would be very limited in the near-term. Given the assumption that borrowing will continue at long maturities, the debt ratios will stay low even in the low-growth scenario. This should dispel any concerns about the country's ability to repay its debt.

\section{Under the high growth scenario, with a higher growth dividend than} conservatively assumed under the baseline, the debt stock ratios would decline rapidly after 2012. If the higher growth dividend materializes, the ensuing strong tax revenues will help contain the deficit, reduce debt, and further expand public expenditures. 


\section{Public Sector Debt Sustainability}

15. The DSA baseline shows that public debt sustainability will be preserved, since the trajectory of total public debt mirrors external debt and yields the same conclusion (Table 4). The PV of total debt-to-GDP peaks at 67 percent of GDP in 2012 and declines thereafter in line with the authorities' commitment in the MTFF to reduce external borrowing from 2012. External borrowing is determining the trajectory of total public debt because net domestic debt (gross domestic debt net of deposits in the BCV and commercial banks) is projected to be kept below 20 percent of GDP. The debt-to-revenue and debt service-torevenue ratios will remain broadly stable.

16. Alternative scenarios and bound tests indicate that debt sustainability indicators remain on paths similar to the external public debt. Only the scenario of an unchanged primary balance from 2010 leads to an unsustainable situation, but it is not relevant since 2010 is an exceptional year, characterized by fiscal stimulus and an accelerated public investment program.

17. Other risks to the debt outlook include a currency mismatch and contingent liabilities of state-owned enterprises. A depreciation of the euro could raise the debt ratios. The current cost to recapitalize Electra and TACV is estimated at 5 percent of GDP.

\section{CONCLUSIONS: DEBT SUSTAINABILITY BROADLY UNCHANGED}

18. The debt sustainability outlook is broadly similar to the 2009 DSA. However, compared to the 2008 DSA, the external and public-sector debt sustainability analysis reveals increased vulnerability as external debt ratios are higher. This reflects an acceleration of public investment, a depreciation of the euro (and thus the CVE) compared to the dollar, and the technical downward revision of the historical nominal GDP numbers.

19. Staff assesses Cape Verde's debt as sustainable. The PV of debt-to-GDP ratio breaches its threshold under half of the stress tests. However, Cape Verde's strong policies and institutions should help ensure that the growth dividends from the accelerated public investments materialize, and help restore buffers in the medium term. Moreover, the long maturities and high concessionality of the existing debt ensure that liquidity risks are low, as shown by debt service indicators.

20. Still, increased debt vulnerabilities call for caution. In particular:

- The current debt assessment is based on several assumptions, including (i) the authorities' commitment to decelerate external borrowing in 2012 and thereafter; (ii) the authorities' capacity to raise revenue in the medium term; (iii) the authorities' ability to contain expenditures; (iv) a rebound in economic growth.

- $\quad$ New borrowing should continue to be mostly at long maturities and on concessional terms. Long maturities will ensure low debt service obligations relative to Cape Verde's export and revenue ratios. If maturities are shorter, and concessionality lower compared to the baseline, the risks to the debt outlook would worsen. 
Table 1.: External Debt Sustainability Framework, Baseline Scenario, 2007-2030 1/ (In percent of GDP, unless otherwise indicated)

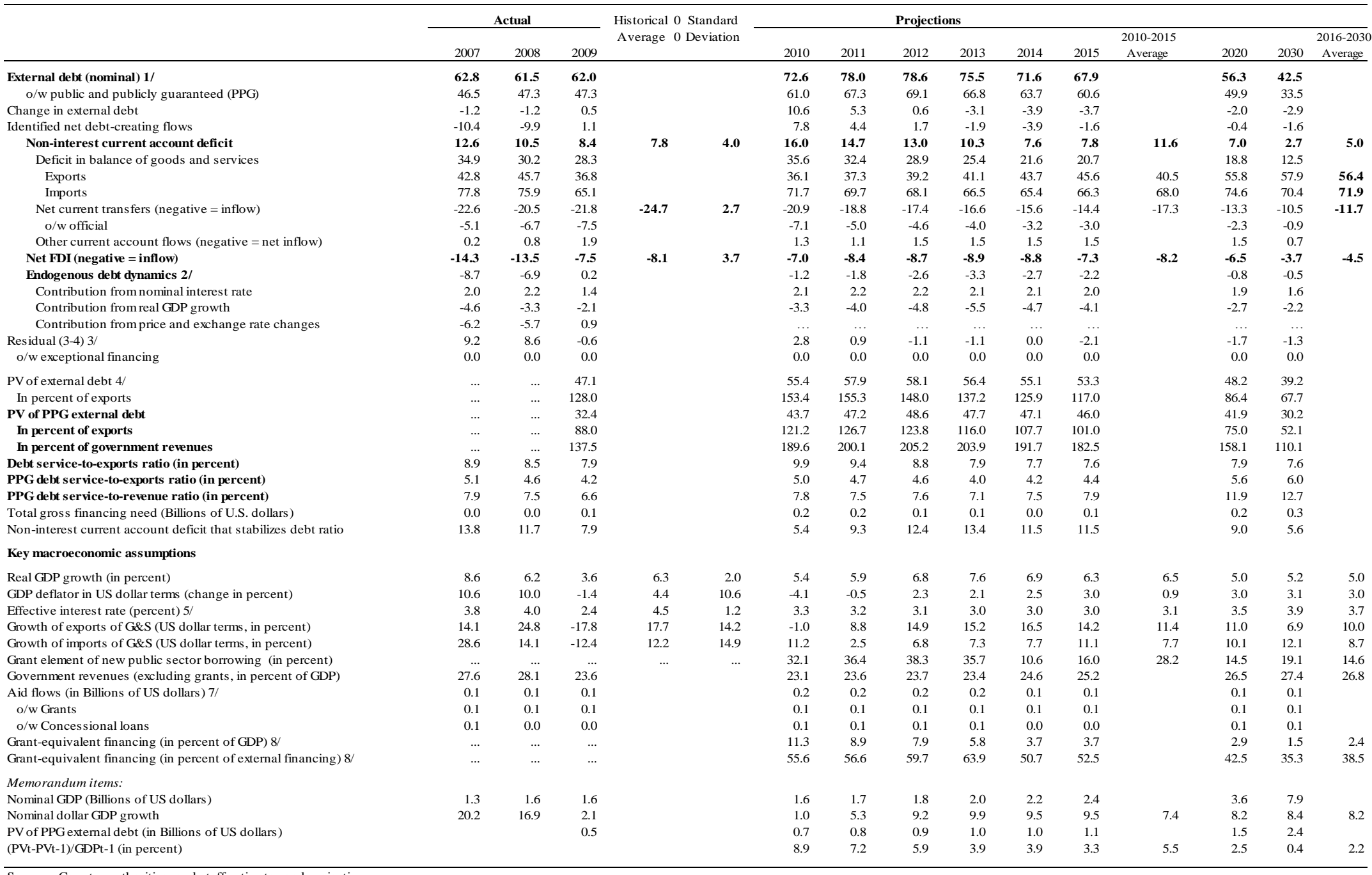

and staff estimates and projections.

1/ Includes both public and private sector external debt.

es previous period debt ratio, with $\mathrm{r}=$ nominal interest rate; $\mathrm{g}=$ real GDP growth rate, and $\rho=$ growth rate of GDP deflator in U.S. dollar terms.

3/ Includes exceptional financing (i.e., changes in arrears and debt relief); changes in gross foreign assets; and valuation adjustments. For projections also includes contribution from price and exchange rate changes.

4/ Assumes that PV of private sector debt is equivalent to its face value.
/ Current-year interest payments divided by previous period debt stock.

6/ Historical averages and standard deviations are generally derived over the past 10 years, subject to data availability.

7/ Defined as grants, concessional loans, and debt relief.

8/ Grant-equivalent financing includes grants provided directly to the government and through new borrowing (difference between the face value and the PV of new debt). 
Table 2.Cape Verde: Sensitivity Analysis for Key Indicators of Public and Publicly Guaranteed External Debt, 2010-2030 (In percent)

\begin{tabular}{|c|c|c|c|c|c|c|c|c|}
\hline & \multicolumn{8}{|c|}{ Projections } \\
\hline & 2010 & 2011 & 2012 & 2013 & 2014 & 2015 & 2020 & 2030 \\
\hline \multicolumn{9}{|l|}{ PV of debt-to GDP ratio } \\
\hline Baseline & 43.7 & 47.2 & 48.6 & 47.7 & 47.1 & 46.0 & 41.9 & 30.2 \\
\hline \multicolumn{9}{|l|}{ A. Alternative Scenarios } \\
\hline A1. Key variables at their historical averages in 2010-2030 1/ & 43.7 & 38.5 & 35.3 & 34.1 & 36.2 & 36.1 & 30.6 & 16.1 \\
\hline A2. New public sector loans on less favorable terms in 2010-2030 2 & 43.7 & 49.8 & 53.3 & 53.3 & 52.3 & 51.9 & 50.2 & 43.5 \\
\hline A3. Low growth Scenario 7/ & 43.8 & 48.0 & 49.9 & 49.7 & 49.7 & 49.3 & 46.4 & 36.0 \\
\hline A4. High Growth Scenario $7 /$ & 43.8 & 46.7 & 47.4 & 46.0 & 44.9 & 43.1 & 37.8 & 24.8 \\
\hline \multicolumn{9}{|l|}{ B. Bound Tests } \\
\hline B1. Real GDP growth at historical average minus one standard deviation in 2011-2012 & 43.7 & 47.8 & 50.5 & 49.6 & 49.0 & 47.9 & 43.5 & 31.4 \\
\hline B2. Export value growth at historical average minus one standard deviation in 2011-2012 3/ & 43.7 & 49.0 & 55.9 & 54.4 & 52.2 & 49.7 & 42.0 & 30.2 \\
\hline B3. US dollar GDP deflator at historical average minus one standard deviation in 2011-2012 & 43.7 & 50.0 & 56.2 & 55.2 & 54.5 & 53.3 & 48.4 & 34.9 \\
\hline B4. Net non-debt creating flows at historical average minus one standard deviation in 2011-2012 4/ & 43.7 & 47.9 & 49.0 & 48.0 & 47.3 & 46.2 & 41.9 & 30.2 \\
\hline B5. Combination of B1-B4 using one-half standard deviation shocks & 43.7 & 44.8 & 47.6 & 47.3 & 47.5 & 47.1 & 44.2 & 31.9 \\
\hline B6. One-time 30 percent nominal depreciation relative to the baseline & 43.7 & 67.5 & 69.5 & 68.3 & 67.4 & 65.9 & 59.9 & 43.2 \\
\hline
\end{tabular}

\section{PV of debt-to-exports ratio}

Baseline

$\begin{array}{llllllll}121.2 & 126.7 & 123.8 & 116.0 & 107.7 & 101.0 & \mathbf{7 5 . 0} & 52.1\end{array}$

A. Alternative Scenarios

A1. Key variables at their historical averages in 2010-2030 1/

A2. New public sector loans on less favorable terms in 2010-2030 2

A3. Low growth Scenario ${ }^{7 /}$

A4. High Growth Scenario ${ }^{7 /}$

\section{B. Bound Tests}

B1. Real GDP growth at historical average minus one standard deviation in 2011-2012

B2. Export value growth at historical average minus one standard deviation in 2011-2012 3/

B3. US dollar GDP deflator at historical average minus one standard deviation in 2011-2012

B4. Net non-debt creating flows at historical average minus one standard deviation in 2011-2012 4/

B5. Combination of B1-B4 using one-half standard deviation shocks

B6. One-time 30 percent nominal depreciation relative to the baseline in $20115 /$

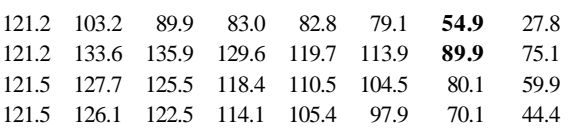

$\begin{array}{llllllll}121.2 & 126.4 & 123.8 & 116.0 & 107.7 & 101.0 & \mathbf{7 5 . 0} & 52.1\end{array}$

$\begin{array}{llllllll}121.2 & 138.1 & 166.4 & 154.4 & 139.4 & 127.3 & \mathbf{8 7 . 9} & 60.8\end{array}$

$\begin{array}{llllllll}121.2 & 126.4 & 123.8 & 116.0 & 107.7 & 101.0 & \mathbf{7 5 . 0} & 52.1\end{array}$

$\begin{array}{llllllll}121.2 & 128.4 & 124.9 & 116.7 & 108.2 & 101.2 & \mathbf{7 5 . 0} & 52.1\end{array}$

$\begin{array}{llllllll}121.2 & 117.2 & 117.2 & 111.3 & 105.0 & 99.8 & \mathbf{7 6 . 6} & 53.3\end{array}$

$\begin{array}{llllllll}121.2 & 126.4 & 123.8 & 116.0 & 107.7 & 101.0 & \mathbf{7 5 . 0} & 52.1\end{array}$

PV of debt-to-revenue ratio

Baseline

$\begin{array}{llllllll}189.6 & 200.1 & 205.2 & 203.9 & 191.7 & 182.5 & \mathbf{1 5 8 . 1} & 110.1\end{array}$

A. Alternative Scenarios

A1. Key variables at their historical averages in 2010-2030 1/

A2. New public sector loans on less favorable terms in 2010-2030 2

A3. Low growth Scenario 7/

A4. High Growth Scenario 7/

\section{B. Bound Tests}

B1. Real GDP growth at historical average minus one standard deviation in 2011-2012

B2. Export value growth at historical average minus one standard deviation in 2011-2012 3/

B3. US dollar GDP deflator at historical average minus one standard deviation in 2011-2012

B4. Net non-debt creating flows at historical average minus one standard deviation in 2011-2012 4/

B5. Combination of B1-B4 using one-half standard deviation shocks

B6. One-time 30 percent nominal depreciation relative to the baseline in 2011 5/

$\begin{array}{llllllll}189.6 & 163.0 & 149.0 & 145.9 & 147.4 & 143.0 & \mathbf{1 1 5 . 8} & 58.8\end{array}$

$\begin{array}{lllllllr}189.6 & 211.0 & 225.2 & 227.7 & 213.0 & 205.8 & \mathbf{1 8 9 . 7} & 158.6\end{array}$

$\begin{array}{llllllll}190.0 & 203.5 & 209.5 & 209.2 & 197.2 & 190.1 & 170.4 & 127.7\end{array}$

$\begin{array}{lllllllll}190.0 & 197.4 & 201.6 & 199.6 & 187.1 & 175.8 & 146.5 & 93.0\end{array}$ 
Table 2.Cape Verde: Sensitivity Analysis for Key Indicators of Public and Publicly Guaranteed External Debt, 2010-2030 (continued) (In percent)

\begin{tabular}{ccccccc}
\hline Projections \\
\cline { 2 - 6 }
\end{tabular}

\section{Debt service-to-exports ratio}

Baseline

\section{A. Alternative Scenarios}

A1. Key variables at their historical averages in 2010-2030 1/ A2. New public sector loans on less favorable terms in 2010-2030 2

A3. Low growth Scenario 7/

A4. High Growth Scenario 7/

\section{B. Bound Tests}

B1. Real GDP growth at historical average minus one standard deviation in 2011-2012 B2. Export value growth at historical average minus one standard deviation in 2011-2012 3/ B3. US dollar GDP deflator at historical average minus one standard deviation in 2011-2012 B4. Net non-debt creating flows at historical average minus one standard deviation in 2011-2012 4/ B5. Combination of B1-B4 using one-half standard deviation shocks

B6. One-time 30 percent nominal depreciation relative to the baseline in $20115 /$

$\begin{array}{rrrrrrrr}5.0 & 4.7 & 4.6 & 4.0 & 4.2 & 4.4 & \mathbf{5 . 6} & 6.0 \\ & & & & & & & \\ 5.0 & 4.5 & 3.7 & 0.4 & -0.7 & -0.5 & \mathbf{3 . 4} & 3.2 \\ 5.0 & 4.7 & 4.9 & 4.7 & 4.5 & 5.3 & \mathbf{6 . 5} & 8.7 \\ 5.0 & 4.8 & 4.6 & 4.0 & 4.3 & 4.4 & 6.0 & 6.7 \\ 5.0 & 4.7 & 4.6 & 3.9 & 4.1 & 4.2 & 5.4 & 5.4\end{array}$

$\begin{array}{llllllll}5.0 & 4.7 & 4.6 & 4.0 & 4.2 & 4.4 & \mathbf{5 . 6} & 6.0 \\ 5.0 & 5.0 & 5.6 & 6.3 & 8.9 & 8.6 & \mathbf{6 . 8} & 7.0 \\ 5.0 & 4.7 & 4.6 & 4.0 & 4.2 & 4.4 & \mathbf{5 . 6} & 6.0 \\ 5.0 & 4.7 & 4.7 & 4.4 & 4.4 & 4.6 & \mathbf{5 . 6} & 6.0 \\ 5.0 & 4.7 & 4.4 & 2.7 & 2.7 & 3.0 & \mathbf{5 . 7} & 6.1 \\ 5.0 & 4.7 & 4.6 & 4.0 & 4.2 & 4.4 & \mathbf{5 . 6} & 6.0\end{array}$

\section{Debt service-to-revenue ratio}

Baseline

\section{A. Alternative Scenarios}

A1. Key variables at their historical averages in 2010-2030 1/ A2. New public sector loans on less favorable terms in 2010-2030 2

A3. Low growth Scenario 7/

A4. High Growth Scenario $7 /$

$\begin{array}{rrrrrrrr}7.8 & 7.1 & 6.2 & 0.6 & -1.3 & -1.0 & \mathbf{7 . 2} & 6.7 \\ 7.8 & 7.5 & 8.1 & 8.3 & 7.9 & 9.5 & \mathbf{1 3 . 7} & 18.3 \\ 7.8 & 7.6 & 7.7 & 7.1 & 7.6 & 8.1 & 12.7 & 14.3 \\ 7.8 & 7.4 & 7.5 & 6.8 & 7.3 & 7.6 & 11.3 & 11.2\end{array}$

\section{B. Bound Tests}

B1. Real GDP growth at historical average minus one standard deviation in 2011-2012

B2. Export value growth at historical average minus one standard deviation in 2011-2012 3/

B3. US dollar GDP deflator at historical average minus one standard deviation in 2011-2012

B4. Net non-debt creating flows at historical average minus one standard deviation in 2011-2012 4/

B5. Combination of B1-B4 using one-half standard deviation shocks

B6. One-time 30 percent nominal depreciation relative to the baseline in $20115 /$

$\begin{array}{rrrrrrrr}7.8 & 7.6 & 7.9 & 7.4 & 7.8 & 8.3 & \mathbf{1 2 . 4} & 13.2 \\ 7.8 & 7.5 & 7.9 & 9.5 & 13.5 & 13.3 & \mathbf{1 2 . 3} & 12.7 \\ 7.8 & 7.9 & 8.8 & 8.2 & 8.7 & 9.2 & \mathbf{1 3 . 8} & 14.6 \\ 7.8 & 7.5 & 7.7 & 7.7 & 7.9 & 8.2 & \mathbf{1 1 . 9} & 12.7 \\ 7.8 & 7.5 & 7.6 & 4.8 & 4.9 & 5.7 & \mathbf{1 2 . 4} & 13.4 \\ 7.8 & 10.7 & 10.9 & 10.1 & 10.8 & 11.4 & \mathbf{1 7 . 0} & 18.1\end{array}$

Memorandum item:

Grant element assumed on residual financing (i.e., financing required above baseline) 6/

$\begin{array}{llllllll}0.0 & 0.0 & 0.0 & 0.0 & 0.0 & 0.0 & \mathbf{0 . 0} & 0.0\end{array}$

Sources: Country authorities; and staff estimates and projections.

1/ Variables include real GDP growth, growth of GDP deflator (in U.S. dollar terms), non-interest current account in percent of GDP, and non-debt creating flows . $2 /$ Assumes that the interest rate on new borrowing is by 2 percentage points higher than in the baseline., while grace and maturity periods are the same as in the baseline.

3/ Exports values are assumed to remain permanently at the lower level, but the current account as a share of GDP is assumed to return to its baseline level after the shock (implicitly assuming an offsetting adjustment in import levels).

4/ Includes official and private transfers and FDI.

5/ Depreciation is defined as percentage decline in dollar/local currency rate, such that it never exceeds 100 percent.

6/ Applies to all stress scenarios except for A2 (less favorable financing) in which the terms on all new financing are as specified in footnote 2.

7/ Assumes growth rates respectively 1 percentage points above and below the baseline in 2011-16. 
Table 3.Cape Verde: Public Sector Debt Sustainability Framework, Baseline Scenario, 2007-2030

(n percent of GDP, unless otherwise indicated)

\begin{tabular}{|c|c|c|c|c|c|c|c|c|c|c|c|c|c|c|c|}
\hline & \multicolumn{3}{|c|}{ Actual } & \multirow[b]{2}{*}{ Average } & \multirow[b]{2}{*}{$\begin{array}{c}\text { Standard } \\
\text { Deviation } \\
\end{array}$} & \multicolumn{4}{|l|}{ Estimate } & \multicolumn{3}{|c|}{ Projections } & \multirow[b]{2}{*}{2020} & \multirow[b]{2}{*}{2030} & \multirow[b]{2}{*}{$\begin{array}{l}2016-30 \\
\text { Average }\end{array}$} \\
\hline & 2007 & 2008 & 2009 & & & 2010 & 2011 & 2012 & 2013 & 2014 & 2015 & $\begin{array}{l}2010-15 \\
\text { Average }\end{array}$ & & & \\
\hline Public sector debt 1/ & 68.0 & 65.0 & 63.9 & & & 80.8 & 86.5 & 87.6 & 84.1 & 79.4 & 74.5 & & 60.5 & 41.3 & \\
\hline $\mathrm{o} / \mathrm{w}$ foreign-currency denominated & 46.5 & 47.3 & 47.3 & & & 62.8 & 67.5 & 69.1 & 66.8 & 63.7 & 60.6 & & 49.9 & 33.5 & \\
\hline Change in public sector debt & -11.6 & -3.1 & -1.1 & & & 16.9 & 5.7 & 1.1 & -3.5 & -4.7 & -4.9 & & -2.1 & -2.7 & \\
\hline Identified debt-creating flows & -12.6 & -2.1 & -0.8 & & & 14.9 & 4.4 & 0.0 & -4.4 & -4.4 & -4.6 & & -2.0 & -2.8 & \\
\hline Primary deficit & -1.0 & -0.3 & 5.3 & 4.2 & 5.1 & 11.8 & 9.6 & 6.4 & 2.5 & 2.0 & 1.3 & 5.6 & 1.8 & -0.3 & 1.3 \\
\hline Revenue and grants & 32.8 & 33.6 & 29.0 & 30.3 & 2.5 & 30.1 & 28.6 & 28.3 & 27.4 & 27.8 & 28.3 & 28.4 & 28.7 & 28.2 & 28.6 \\
\hline of which: grants & 5.2 & 5.5 & 5.4 & & & 7.1 & 5.0 & 4.6 & 4.0 & 3.2 & 3.0 & & 2.2 & 0.8 & \\
\hline Primary (noninterest) expenditure & 31.8 & 33.3 & 34.3 & 34.5 & 3.9 & 42.0 & 38.2 & 34.7 & 29.9 & 29.8 & 29.6 & 34.0 & 30.5 & 27.9 & 29.9 \\
\hline Automatic debt dynamics & -10.3 & -1.1 & -6.2 & & & 3.2 & -5.0 & -6.4 & -7.1 & -6.4 & -6.0 & & -3.8 & -2.5 & \\
\hline Contribution from interest rate/growth differential & -6.5 & -4.0 & -1.9 & & & -2.9 & -4.4 & -5.8 & -6.7 & -6.0 & -5.4 & & -3.3 & -2.1 & \\
\hline of which: contribution from average real interest rate & -0.2 & 0.0 & 0.3 & & & 0.4 & 0.0 & -0.3 & -0.5 & -0.6 & -0.7 & & -0.3 & 0.1 & \\
\hline of which: contribution from real GDP growth & -6.3 & -4.0 & -2.2 & & & -3.3 & -4.5 & -5.5 & -6.2 & -5.4 & -4.7 & & -3.0 & -2.2 & \\
\hline Contribution from real exchange rate depreciation & -3.8 & 2.9 & -4.3 & & & 6.1 & -0.6 & -0.6 & -0.3 & -0.4 & -0.6 & & & $\ldots$ & \\
\hline Other identified debt-creating flows & -1.4 & -0.7 & 0.1 & & & -0.1 & -0.1 & 0.0 & 0.1 & 0.1 & 0.1 & & 0.0 & 0.0 & \\
\hline Privatization receipts (negative) & -1.4 & -0.7 & 0.1 & & & -0.1 & -0.1 & 0.0 & 0.1 & 0.1 & 0.1 & & 0.0 & 0.0 & \\
\hline Recognition of implicit or contingent liabilities & 0.0 & 0.0 & 0.0 & & & 0.0 & 0.0 & 0.0 & 0.0 & 0.0 & 0.0 & & 0.0 & 0.0 & \\
\hline Debt relief (HIPC and other) & 0.0 & 0.0 & 0.0 & & & 0.0 & 0.0 & 0.0 & 0.0 & 0.0 & 0.0 & & 0.0 & 0.0 & \\
\hline Other (specify, e.g. bank recapitalization) & 0.0 & 0.0 & 0.0 & & & 0.0 & 0.0 & 0.0 & 0.0 & 0.0 & 0.0 & & 0.0 & 0.0 & \\
\hline Residual, including asset changes & 1.0 & -0.9 & -0.3 & & & 2.0 & 1.3 & 1.1 & 0.9 & -0.3 & -0.3 & & -0.1 & 0.1 & \\
\hline \multicolumn{16}{|l|}{ Other Sustainability Indicators } \\
\hline PV of public sector debt & 21.5 & 17.7 & 48.9 & & & 61.7 & 66.3 & 67.1 & 65.0 & 62.8 & 59.9 & & 52.5 & 38.0 & \\
\hline $\mathrm{o} / \mathrm{w}$ foreign-currency denominated & 0.0 & 0.0 & 32.4 & & & 43.7 & 47.2 & 48.6 & 47.7 & 47.1 & 46.0 & & 41.9 & 30.2 & \\
\hline o/w external & $\ldots$ & $\ldots$ & 32.4 & & & 43.7 & 47.2 & 48.6 & 47.7 & 47.1 & 46.0 & & 41.9 & 30.2 & \\
\hline PV of contingent liabilities (not included in public sector debt) & $\ldots$ & & $\ldots$ & & & $\ldots$ & $\ldots$ & & & $\ldots$ & $\ldots$ & & & $\ldots$ & \\
\hline Gross financing need $2 /$ & 4.6 & 4.7 & 11.1 & & & 17.2 & 14.8 & 11.8 & 7.9 & 8.7 & 8.2 & & 9.0 & 8.5 & \\
\hline $\mathrm{PV}$ of public sector debt-to-revenue and grants ratio (in percent) & 65.6 & 52.6 & 168.7 & & & 204.9 & 231.9 & 237.4 & 237.5 & 226.0 & 211.9 & & 182.9 & 134.6 & \\
\hline $\mathrm{PV}$ of public sector debt-to-revenue ratio (in percent) & 78.0 & 62.9 & 207.6 & & & 267.6 & 280.9 & 283.5 & 277.9 & 255.7 & 237.4 & & 198.3 & 138.7 & \\
\hline o/w external $3 /$ & & & 137.5 & & & 189.6 & 200.1 & 205.2 & 203.9 & 191.7 & 182.5 & & 158.1 & 110.1 & \\
\hline Debt service-to-revenue and grants ratio (in percent) $4 /$ & 17.0 & 14.8 & 20.2 & & & 17.9 & 18.4 & 19.1 & 19.8 & 24.1 & 24.1 & & 25.2 & 31.2 & \\
\hline Debt service-to-revenue ratio (in percent) $4 /$ & 20.3 & 17.7 & 24.8 & & & 23.4 & 22.3 & 22.8 & 23.2 & 27.3 & 27.0 & & 27.4 & 32.1 & \\
\hline Primary deficit that stabilizes the debt-to-GDP ratio & 10.6 & 2.8 & 6.4 & 6.6 & 3.9 & -5.1 & 3.8 & 5.3 & 6.0 & 6.6 & 6.2 & 3.8 & 3.9 & 2.4 & 3.5 \\
\hline \multicolumn{16}{|l|}{ Key macroeconomic and fiscal assumptions } \\
\hline Real GDP growth (in percent) & 8.6 & 6.2 & 3.6 & 6.3 & 2.0 & 5.4 & 5.9 & 6.8 & 7.6 & 6.9 & 6.3 & 6.5 & 5.0 & 5.2 & 5.0 \\
\hline Average nominal interest rate on forex debt (in percent) & 1.1 & 1.1 & 0.0 & 1.2 & 0.5 & 0.7 & 0.8 & 0.9 & 0.9 & 0.9 & 0.9 & 0.9 & 1.3 & 2.2 & 1.6 \\
\hline Average real interest rate on domestic debt (in percent) & 2.5 & 1.9 & 0.6 & 4.0 & 2.8 & 2.7 & 1.7 & 0.2 & -0.3 & -0.6 & -0.6 & 0.5 & -0.5 & -0.6 & -0.6 \\
\hline Real exchange rate depreciation (in percent, + indicates depreciation) & -8.0 & 6.7 & -9.4 & -1.8 & 12.8 & 13.5 & $\ldots$ & & & $\ldots$ & $\ldots$ & $\ldots$ & & $\ldots$ & $\ldots$ \\
\hline Inflation rate (GDP deflator, in percent) & 1.4 & 2.5 & 4.2 & 1.2 & 2.3 & 3.3 & 2.8 & 2.8 & 2.6 & 3.0 & 3.0 & 2.9 & 3.0 & 3.1 & 3.0 \\
\hline Growth of real primary spending (deflated by GDP deflator, in percent) & 0.0 & 0.1 & 0.1 & 0.1 & 0.2 & 0.3 & 0.0 & 0.0 & -0.1 & 0.1 & 0.1 & 0.0 & 0.1 & 0.0 & 0.0 \\
\hline Grant element of new external borrowing (in percent) & $\ldots$ & $\ldots$ & $\ldots$ & & .. & 32.1 & 36.4 & 38.3 & 35.7 & 10.6 & 16.0 & 28.2 & 14.5 & 19.1 & $\ldots$ \\
\hline
\end{tabular}

Sources: Country authorities; and staff estimates and projections.
1 [Indicate coverage of public sector, e.g., general government or nonfinancial public sector. Also whether net or gross debt is used.]

$2 /$ Gross financing need is defined as the primary deficit plus debt service plus the stock of short-term debt at the end of the last period.

$3 /$ Revenues excluding grants.

4/ Debt service is defined as the sum of interest and amortization of medium and long-term debt.

5/Historical averages and standard deviations are generally derived over the past 10 years, subject to data availability. 
Table 4.Cape Verde: Sensitivity Analysis for Key Indicators of Public Debt 2010-2030



PV of Debt-to-GDP Ratio

Baseline

\section{A. Alternative scenarios}

A1. Real GDP growth and primary balance are at historical averages A2. Primary balance is unchanged from 2010

A3. Permanently lower GDP growth $1 /$

B. Bound tests

B1. Real GDP growth is at historical average minus one standard deviations in 2011-2012 B2. Primary balance is at historical average minus one standard deviations in 2011-2012 B3. Combination of B1-B2 using one half standard deviation shocks B4. One-time 30 percent real depreciation in 2011

B5. 10 percent of GDP increase in other debt-creating flows in 2011

PV of Debt-to-Revenue Ratio 2/

Baseline

\section{A. Alternative scenarios}

A1. Real GDP growth and primary balance are at historical averages A2. Primary balance is unchanged from 2010

A3. Permanently lower GDP growth $1 /$

B. Bound tests

B1. Real GDP growth is at historical average minus one standard deviations in 2011-2012 B2. Primary balance is at historical average minus one standard deviations in 2011-2012

B3. Combination of B1-B2 using one half standard deviation shocks

B4. One-time 30 percent real depreciation in 2011

B5. 10 percent of GDP increase in other debt-creating flows in 2011

Debt Service-to-Revenue Ratio 2/

Baseline

$\begin{array}{llllllll}62 & 66 & 67 & 65 & 63 & 60 & 52 & 38\end{array}$

$\begin{array}{llllllll}62 & 61 & 60 & 61 & 61 & 62 & 64 & 73\end{array}$

$\begin{array}{llllllll}62 & 69 & 76 & 83 & 91 & 98 & 138 & 214\end{array}$

$\begin{array}{llllllll}62 & 67 & 68 & 67 & 66 & 64 & 62 & 67\end{array}$

A. Alternative scenarios

A1. Real GDP growth and primary balance are at historical averages A2. Primary balance is unchanged from 2010

A3. Permanently lower GDP growth 1 /

\section{B. Bound tests}

B1. Real GDP growth is at historical average minus one standard deviations in 2011-2012 B2. Primary balance is at historical average minus one standard deviations in 2011-2012

B3. Combination of B1-B2 using one half standard deviation shocks

B4. One-time 30 percent real depreciation in 2011

B5. 10 percent of GDP increase in other debt-creating flows in 2011 $\begin{array}{llllllll}205 & 232 & 237 & 238 & 226 & 212 & 183 & 135\end{array}$

$\begin{array}{llllllll}205 & 212 & 211 & 221 & 221 & 218 & 224 & 259 \\ 205 & 241 & 268 & 304 & 327 & 348 & 482 & 757\end{array}$



$\begin{array}{llllllll}205 & 237 & 252 & 256 & 248 & 237 & 225 & 211 \\ 205 & 232 & 249 & 250 & 238 & 224 & 195 & 149 \\ 205 & 223 & 233 & 236 & 227 & 215 & 197 & 169 \\ 205 & 291 & 291 & 289 & 276 & 260 & 229 & 196 \\ 205 & 270 & 275 & 275 & 262 & 247 & 216 & 167\end{array}$

$\begin{array}{llllllll}18 & 18 & 19 & 20 & 24 & 24 & 25 & 31\end{array}$

$\begin{array}{llllllll}18 & 18 & 18 & 19 & 19 & 17 & 30 & 53\end{array}$

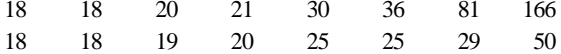

Sources: Country authorities; and staff estimates and projections.

1/ Assumes that real GDP growth is at baseline minus one standard deviation divided by the square root of the length of the projection period.

2/ Revenues are defined inclusive of grants . 
Figure 1. Cape Verde: Indicators of Public and Publicly Guaranteed External Debt under Alternatives Scenarios, 2010-2030 1/
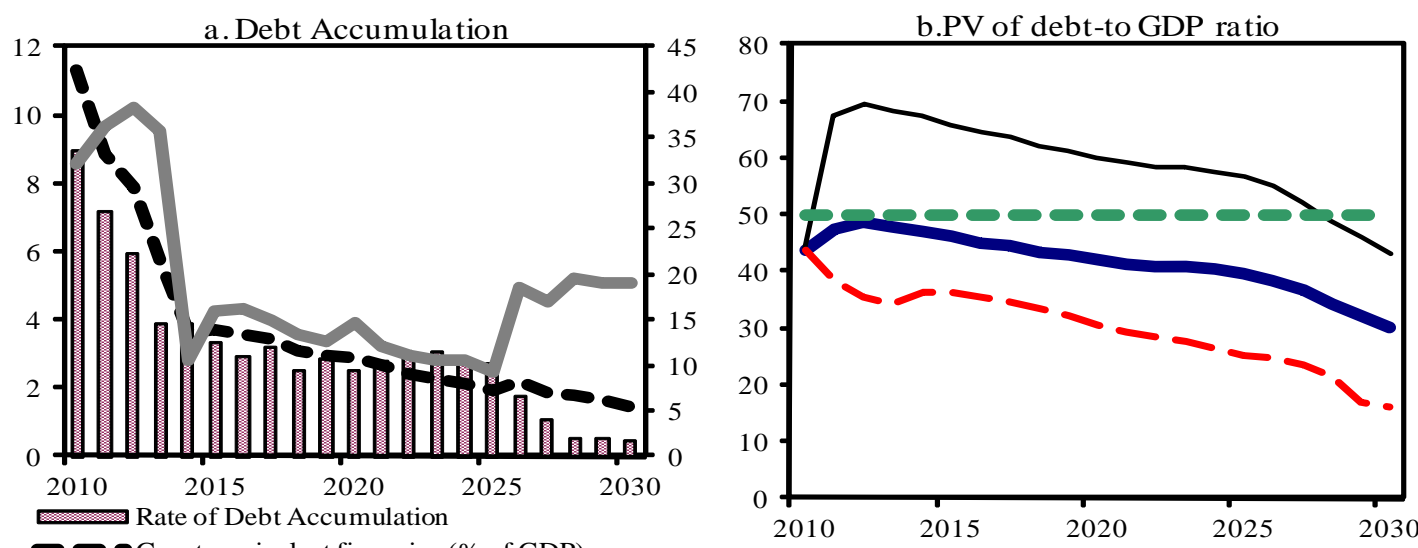

- Grant-equivalent financing (\% of GDP)

Grant element of new borrowing (\% right scale)
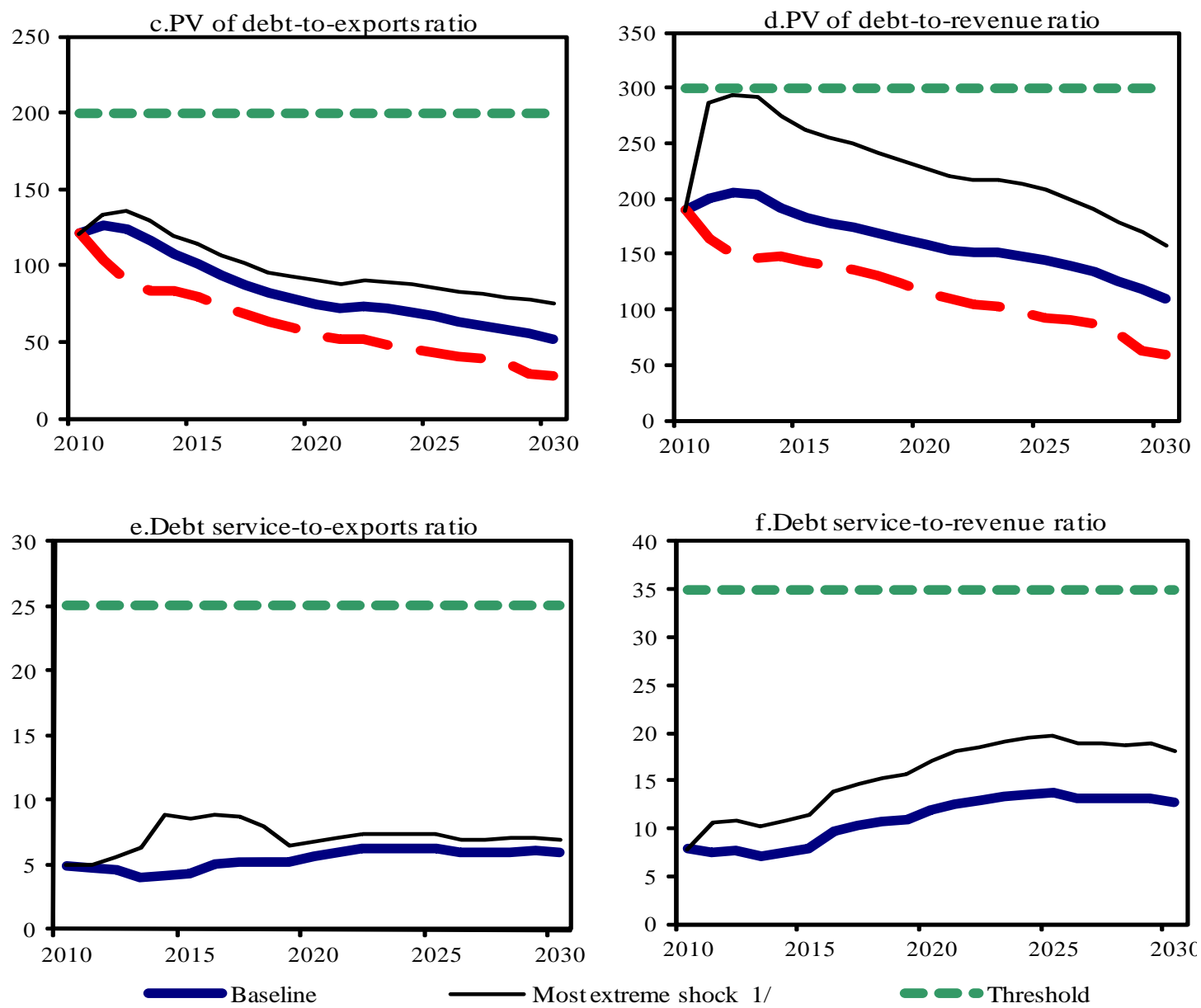

Sources: Country authorities; and staff estimates and projections.

$1 /$ The most extreme stress test is the test that yields the highest ratio in 2020. In figure b. it corresponds to a One-time depreciation shock; in c. to a Terms shock; in d. to a One-time depreciation shock; in e. to a Exports shock and in figure f. to a One-time depreciation shock 
Figure 2.Cape Verde: Indicators of Public Debt Under Alternative Scenarios, 2010-2030 1/


Sources: Country authorities; and staff estimates and projections.

1/ The most extreme stress test is the test that yields the highest ratio in 2020.

2/ Revenues are defined inclusive of grants. 
Annex I. CAPE Verde-Fund Relations

(as of October 31, 2010)

I. Membership Status: Joined: November 20, 1978;

Article VIII

II. General Resources Account:

SDR Million

\%Quota

Quota

9.60

100.00

Fund holdings of currency

9.59

99.93

Reserve Position

0.02

0.17

Holdings Exchange Rate

\section{SDR Department:}

Net cumulative allocation

Holdings

IV. Outstanding Purchases and Loans:

ECF Arrangements
SDR Million

9.17

7.11

SDR Million

5.93
\%Allocation

100.00

77.52

\%Quota

61.81

\section{Latest Financial Arrangements:}

\begin{tabular}{|c|c|c|c|c|}
\hline Type & $\begin{array}{c}\text { Date of } \\
\text { Arrangement } \\
\end{array}$ & $\begin{array}{l}\text { Expiration } \\
\text { Date }\end{array}$ & $\begin{array}{l}\text { Amount Approved } \\
\text { (SDR Million) }\end{array}$ & $\begin{array}{l}\text { Amount Drawn } \\
\text { (SDR Million) }\end{array}$ \\
\hline $\mathrm{ECF}^{1 /}$ & Apr 10,2002 & Jul $31 \overline{, 2005}$ & 8.64 & 8.64 \\
\hline Stand-By & Feb 20, 1998 & $\operatorname{Mar} 15,2000$ & 2.50 & 0.00 \\
\hline
\end{tabular}

\section{Projected Payments to Fund ${ }^{2}$}

(SDR Million; based on existing use of resources and present holdings of SDRs):

\begin{tabular}{lccccr} 
& \multicolumn{5}{c}{ Forthcoming } \\
\cline { 2 - 6 } & $\underline{2010}$ & $\underline{2011}$ & $\underline{2012}$ & $\underline{2013}$ & $\underline{2014}$ \\
Principal & 0.37 & 1.73 & 1.61 & 1.24 & 0.74 \\
Charges/Interest & 0.00 & 0.01 & 0.02 & 0.01 & 0.01 \\
Total & 0.37 & 1.74 & 1.62 & 1.25 & 0.75
\end{tabular}

${ }^{2}$ When a member has overdue financial obligations outstanding for more than three months, the amount of such arrears will be shown in this section.

VII. Implementation of HIPC Initiative: Not Applicable

VIII. Implementation of Multilateral Debt Relief Initiative (MDRI): Not Applicable IX. Safeguards Assessments

In response to a request from the Bank of Cape Verde's (BCV), a voluntary safeguard assessment with respect to the Policy Support Instrument (PSI) approved on July 31, 2006 was completed in 2008. The previous safeguards assessment of the BCV, which was completed in December 2002, concluded that substantial risks may have existed at the time 
in the bank's financial reporting framework, its internal audit mechanism, and system of internal controls. The 2008 assessment concluded that, while the BCV has taken some measures recommended in the 2002 assessment, there are still significant outstanding vulnerabilities, notably in the system of internal control, internal audit, and transparency and publication of the financial statements.

\section{Exchange Arrangements}

The de facto and de jure exchange rate arrangement of Cape Verde is a conventional fixed peg. The escudo has been pegged to the euro at a rate of CVE 110.265 per $€ 1$ since January 4, 1999. Cape Verde accepted the obligations of Article VIII of the Articles of Agreement effective July 1, 2004. It maintains an exchange system that is free of restrictions on the making of payments and transfers for current international transactions.

\section{Previous Article IV Consultation and PSI Reviews}

Discussions for the 2006 Article IV consultation and on a medium-term economic program that could be supported by a three-year PSI were held in Praia April 28-May 16, 2006. The Executive Board concluded the Article IV consultation and approved the request for a PSI on July 31, 2006 (Country Report No. 06/334); the first PSI review on January 19, 2007 (Country Report No. 07/44); the second PSI review on May 7, 2007 (Country Report No. 07/223); the third PSI review on December 21, 2007 (Country Report 08/37); the 2008 Article IV consultation and the fourth PSI review on June 30, 2008 (Country Report 08/248); the fifth PSI review on December 22, 2008 (Country Report 09/14); the sixth PSI review on June 19, 2009 (Country Report 09/198); the seventh PSI review on December 18, 2009 (Country Report 09/328); and the eight PSI review on July 19, 2010 (Country Report 10/218).

\section{Technical Assistance}

Since 1985 the Fund has provided technical assistance to the BCV, the Ministry of Finance, and more recently the National Institute of Statistics in several areas: (i) MCM provided technical assistance to the BCV on organization and methods, management of external debt, monetary and banking statistics, accounting, credit, foreign exchange operations, management of public debt, and the separation of the functions of the Bank, as well as on the choice of exchange rate regime. BCV has also received technical assistance from STA on monetary and balance of payments statistics. (ii) The Ministry of Finance has received technical assistance from FAD on organization and budgetary procedures, budgeting, tax policy, and tax administration; from STA on fiscal accounting; and from LEG on tax legislation. (iii) The National Institute of Statistics has received technical assistance in national accounts and price statistics. Cape Verde is a participant in STA's GDDS Regional Project for Lusophone Africa, and its metadata were posted on the DSBB in February 2004. It is now receiving technical assistance to implement the GDDS plan for improvement.

Most recently, technical assistance has been provided in the following areas: 


\section{FAD}

- June 2004, visit to help the authorities move to a VAT, rationalize the import tariff, and overhaul the domestic indirect tax system. Many visits and a two-year resident advisor have gone into this effort.

- October 2004, mission to review tax administration, including VAT implementation, and a mission to help assess tax exemptions and incentives.

- $\quad$ September 2005, mission to assess tax exemptions and incentives.

- June-July 2008, mission to review and rationalize tax exemptions.

\section{STA}

- $\quad$ National accounts (November 2003 and January-February 2006), balance of payments statistics (February 2004), government finance statistics (March 2004, April 2006, February-March 2007), price statistics (June 2004, May-June 2006, October 2007), and monetary statistics and reporting (March 2007), government finance statistics (August 2008).

\section{MCM}

- $\quad$ Accounting, financial sector regulation, monetary operations and liquidity management (April and May 2004); banking supervision, liquidity management, exchange regime and reserves management (November 2005, March-April 2006, June 2006, November 2006, July 2007).

- $\quad$ Macro-prudential indicators (March 2008).

- $\quad$ Financial Sector Assessment Program (FSAP) (November 2008) a various follow up missions on banking supervision (January, March and April 2009).

- Joint IMF-World Bank on debt management (DeMPA, February 2009) and on Medium-Term Debt Strategy (MTDS) (August-September 2009)

\section{LEG}

- $\quad$ Tax legislation (several missions October 2006-March 2008).

- $\quad$ AML/CFT initial assessment (March 2007), and legal drafting (March 2008).

\section{Resident Representative: None.}


ANNEX II. CAPE VERde-JoInt IMF-WorLd BANK MANAgement ACTION PlanIMPLEMENTATION MATRIX

\begin{tabular}{|c|c|c|c|}
\hline Title & Products & $\begin{array}{l}\text { Provisional } \\
\text { Timing of } \\
\text { Missions } \\
\end{array}$ & $\begin{array}{l}\text { Expected Delivery } \\
\text { Date }\end{array}$ \\
\hline \multicolumn{4}{|c|}{ A. Mutual information on relevant work programs } \\
\hline $\begin{array}{l}\text { Bank work } \\
\text { program in } \\
\text { the next } 12 \\
\text { months }\end{array}$ & $\begin{array}{l}\text { Lending: } \\
\text { - PRSC-6 (IDA) } \\
\text { - Roads Project-2 (IDA) } \\
\text { - SME Technical Assistance and } \\
\text { Capacity Building Operation (IDA) } \\
\text { - Energy Operation (IBRD) } \\
\text { - Trust Fund for Statistical } \\
\text { Capacity Building (TFSCB) - Grant } \\
\text { Analytical and advisory activities: } \\
\text { - Poverty assessment } \\
\text { - Country Economic Memorandum } \\
\text { - Public-Private Infrastructure } \\
\text { Advisory Facility (PPIAF)/TA } \\
\text { proposal on road sector is under } \\
\text { revision } \\
\text { - PPIAF/TA on the energy sector } \\
\text { (develop strategic/recovery plan for } \\
\text { Electra; Electra management model) } \\
\text { - Skills Development/Labor market } \\
\text { study }\end{array}$ & $\begin{array}{l}\text { May/Sep. } 2010 \\
\text { October } 2009 \\
\text { (ongoing) } \\
\text { November } 2009 \\
\text { November } 2009 \\
\text { Dec } 2009 \\
\text { Dec. } 2009 \\
\text { May/Sep } 2010 \\
\text { Nov./Dec. } 2009\end{array}$ & $\begin{array}{l}\text { Q2 FY11 } \\
\text { Q2 FY11 (Dec 2010) } \\
\text { Q4 FY10 (April 2010) } \\
\text { Q3 FY11 } \\
\text { Approved in May 27, } \\
2010 \\
\text { Jul. } 2010 \\
\text { Q4 FY11 } \\
\text { April/May } 2010\end{array}$ \\
\hline
\end{tabular}


Cape Verde: Joint Management Action Plan-Implementation Matrix (concluded)

\begin{tabular}{|l|l|l|l|}
\hline Title & Products & \multicolumn{1}{c|}{$\begin{array}{c}\text { Provisional Timing of } \\
\text { Missions }\end{array}$} & $\begin{array}{c}\text { Expected Delivery } \\
\text { Date }\end{array}$ \\
\hline \multicolumn{3}{|c|}{ A. Mutual information on relevant work programs } \\
\hline $\begin{array}{l}\text { IMF work program } \\
\text { in next 12 months }\end{array}$ & $\begin{array}{l}\text { PSI program, 1st } \\
\text { review }\end{array}$ & May-June 2011 & $\begin{array}{l}\text { Board meeting July } \\
2011\end{array}$ \\
\cline { 2 - 4 } & $\begin{array}{l}\text { PSI program, 2nd } \\
\text { review }\end{array}$ & November 2011 & $\begin{array}{l}\text { Board meeting } \\
\text { January 2012 }\end{array}$ \\
\cline { 2 - 4 } & $\begin{array}{l}\text { Technical assistance } \\
\text { on monetary } \\
\text { transmission } \\
\text { mechanism }\end{array}$ & November 2010 & \\
\cline { 2 - 5 } & $\begin{array}{l}\text { Technical assistance } \\
\text { on monetary } \\
\text { operations }\end{array}$ & November 2010 & \\
\hline & \multicolumn{2}{|l|}{ B. Agreement on joint products and missions } \\
\hline $\begin{array}{l}\text { Joint products in } \\
\text { next 12 months }\end{array}$ & $\begin{array}{l}\text { Technical assistance } \\
\text { on debt management }\end{array}$ & January-March 2010 & $\begin{array}{l}\text { End FY2010 (possible } \\
\text { second phase of TA in } \\
\text { FY11) }\end{array}$ \\
\hline
\end{tabular}




\section{ANNEX III. CAPE VERdE-STATISTICS ISSUES}

1. Data provision has some shortcomings, but is broadly adequate for surveillance. There is a need for substantial improvements in the areas of government finances, national accounts, and balance of payments. The country has participated in the GDDS since February 2004. Cape Verde's plans for improvements of its statistical system are posted on the Fund's DSBB; the metadata of the monetary and financial sector were updated in August 2007. The country has benefited from STA technical assistance under the GDDS project for Lusophone Africa.

2. The authorities are taking steps to strengthen statistics, but the statistical system still suffers from a shortage of financial and human resources. A comprehensive master plan has been developed under the direction of the National Statistical Institute (INE), which assesses the need for upgrading the agencies that constitute the statistical system and outlines steps to broaden and improve all areas of statistics. The country's development partners have already committed a substantial part of the estimated US\$15 million required to implement the plan through 2010.

\section{Real sector statistics}

3. Although some improvements have been made to the national accounts, significant weaknesses remain, including a lack of reliable demand-side GDP. The timeliness of the data has not been improved, with the INE releasing production and expenditure-based GDP data for 2005-07 in 2010 and providing production-based GDP estimates for 2008 to the AFR mission in September 2010. However, the lag is still lengthy by international standards. In addition, the base year for the constant price estimates is outdated. INE is receiving technical assistance from Brazil and Spain in developing business indicators and national accounts.

4. INE is currently working on a complete overhaul of the national accounts. It is updating the national accounts benchmark and base years from 1980 to 2002 and adopting the 1993 SNA. The objective is timely compilation of GDP by industry and expenditure categories at current and constant prices as well as institutional sector accounts.

5. INE will also introduce Tourism Satellite Accounts. These accounts will measure upstream linkages between the tourism sector and the rest of the economy and the import content of tourism exports.

6. Full implementation of the 1993 SNA would require a substantial improvement in source data collection, for which capacity is currently lacking. The 2006 STA mission found a critical need to improve the timeliness and accuracy of national accounts source data, in particular the business survey. The capacity of INE staff working on national accounts was found to be overstretched; they assess in detail and correct individual source data entries- 
something not undertaken in most countries by national accounts compilers. The statistical master plan will address many of these challenges.

7. A revamped CPI with new methodology was launched in February 2008. The previous official CPI was based on weights dating back to 1989 , four years before imports were liberalized. Import liberalization considerably changed consumption patterns. INE has had assistance from the National Statistics Institute of Portugal and benefited from STA CPI missions in May-June, 2006, October 2006, and October 2007, to support the introduction and dissemination of the revised CPI. The new index has new weights and an updated commodity basket.

8. INE publishes labor market statistics only on annual basis with long delays. Recently, it published annual unemployment data, which is comparable to EUROSTAT and AFRISTAT. INE is in the process of finalizing the 2010 census. There is no data on wages in different sectors.

\section{Government finance statistics}

9. Fiscal data have been improved. Benefiting from TA under the GDDS project for Lusophone Africa, the Government Finance Statistics (GFS) compilation system is being upgraded. Most deposits previously held with commercial banks have been consolidated at the central bank. SIGOF, the Integrated Online Budget Management System, has been expanded to cover all semi-autonomous institutes and most municipalities by the end of 2007. The authorities have started reporting GFS for publication in the GFS Yearbook.

10. However quality is a serious concern. The fiscal accounts are subject to statistical discrepancies, flows and stocks are not always consistent. Significant delay in donor reporting of project financing also affects the accuracy of fiscal data. Despite the recent revision of external debt data, significant weaknesses affect preparation of debt sustainability analyses, especially regarding the PPG debt of state owned enterprises. Multilateral debt statistics regularly differ from data received by creditors, and debt service projections cannot be reconciled with the debt stock.

\section{Monetary and financial statistics}

\section{The quality of monetary and financial statistics is adequate, in terms of both} accuracy and timeliness. There has been some delays in publishing the BCV quarterly economic and financial statistical bulletin due to the migration of the banking sector to the International Financial Reporting Standards (IFRS).An STA mission undertaken in March 2007 helped Banco de Cabo Verde (BCV) to finalize the standardized report forms (SRFs) for reporting monetary statistics to STA. SRF-based monetary data have been published in the IFS Supplement since June 2007. These data are fully aligned with the recommendations of the Monetary and Financial Statistics Manual. The integrated monetary database that meets STA, AFR, and BCV statistical needs is now in place. Currently, there are gaps in 
tracking the source and direction of changes in emigrant deposits, which cause difficulties in assessing their interest sensitivity and gauging the appropriateness of the monetary policy stance.

\section{External sector statistics}

12. With technical assistance from STA, the accuracy, periodicity, and timeliness of balance of payments statistics compiled by BCV have continued to improve. A greater use of surveys, combined with the International Transactions Reporting System implemented by the $\mathrm{BCV}$, has permitted a significant expansion of data sources and statistical coverage, which to a large extent follow the recommendations of the 5th edition of the Balance of Payments Manual. However, gaps in the tracking of large external flows-notably FDI, emigrant deposits, and remittances - remains. Dissemination of quarterly BOP data on the BCV website has been regular and in 2007 the BCV resumed regular and timely transmission of these data to STA for publication in International Financial Statistics and in the Balance of Payments Statistics Yearbook. However, the BCV does not compile an International Investment Position statement. 


\section{Cape Verde: Common Indicators Required for Surveillance}

(As of October 15, 2010)

\begin{tabular}{|c|c|c|c|c|c|}
\hline & $\begin{array}{c}\text { Date of } \\
\text { Latest } \\
\text { Observation }\end{array}$ & $\begin{array}{c}\text { Date } \\
\text { Received }\end{array}$ & $\begin{array}{l}\text { Frequency } \\
\text { of Data }^{7}\end{array}$ & $\begin{array}{l}\text { Frequency } \\
\text { of } \\
\text { Reporting }^{7}\end{array}$ & $\begin{array}{l}\text { Frequency } \\
\text { of } \\
\text { Publication }^{7}\end{array}$ \\
\hline Exchange Rates & $05 / 31 / 10$ & $06 / 06 / 10$ & D & D & D \\
\hline $\begin{array}{l}\text { International Reserve Assets } \\
\text { and Reserve Liabilities of the } \\
\text { Monetary Authorities }\end{array}$ & July 10 & Sept. 10 & D & W & M \\
\hline Reserve/Base Money & July 10 & Sept. 10 & $\mathrm{D}$ & W & M \\
\hline Broad Money & July 10 & Sept. 10 & M & $M$ & M \\
\hline Central Bank Balance Sheet & July 10 & Sept. 10 & $\mathrm{D}$ & W & $M$ \\
\hline $\begin{array}{l}\text { Consolidated Balance Sheet of } \\
\text { the Banking System }\end{array}$ & July 10 & Sept. 10 & M & M & M \\
\hline Interest Rates $^{2}$ & Aug. 10 & Sept. 10 & $M$ & $M$ & $M$ \\
\hline Consumer Price Index & April 10 & May 10 & M & M & M \\
\hline $\begin{array}{l}\text { Revenue, Expenditure, Balance } \\
\text { and Composition of Financing } \\
\text { - General Government }\end{array}$ & $\mathrm{N} / \mathrm{A}$ & $\mathrm{N} / \mathrm{A}$ & $N / A$ & $\mathrm{~N} / \mathrm{A}$ & $N / A$ \\
\hline $\begin{array}{l}\text { Revenue, Expenditure, Balance } \\
\text { and Composition of Financing }{ }^{3}- \\
\text { Central Government }\end{array}$ & June 10 & August-10 & $Q$ & $Q$ & $Q$ \\
\hline $\begin{array}{l}\text { Stocks of Central Government } \\
\text { and Central Government- } \\
\text { Guaranteed Debt }{ }^{5}\end{array}$ & Dec. 09 & May 10 & $A$ & $A$ & $A$ \\
\hline $\begin{array}{l}\text { External Current Account } \\
\text { Balance }\end{array}$ & June 10 & Sept. 10 & $Q$ & $Q$ & $Q$ \\
\hline $\begin{array}{l}\text { Exports and Imports of Goods } \\
\text { and Services }\end{array}$ & June 10 & Sept. 10 & $Q$ & $Q$ & $Q$ \\
\hline GDP/GNP & 2007 & March 10 & $A$ & $A$ & $A$ \\
\hline Gross External Debt & Dec. 09 & May 10 & $A$ & A & A \\
\hline $\begin{array}{l}\text { International Investment } \\
\text { Position }^{6}\end{array}$ & 2007 & Aug. 08 & A & $A$ & $A$ \\
\hline
\end{tabular}

${ }^{1}$ Includes reserve assets pledged or otherwise encumbered as well as net derivative positions.

${ }^{2}$ Both market-based and officially-determined, including discount rates, money market rates, rates on treasury bills, notes and bonds.

${ }^{3}$ Foreign, domestic bank, and domestic nonbank financing.

${ }^{4}$ The general government consists of the central government (budgetary funds, extra budgetary funds, and social security funds) and state and local governments.

${ }^{5}$ Including currency and maturity composition.

${ }^{6}$ Includes external gross financial asset and liability positions vis-à-vis nonresidents.

${ }^{7}$ Daily (D), weekly (W), monthly (M), quarterly (Q), annually (A), irregular (I); and not available (NA). 


\section{INTERNATIONAL MONETARY FUND}

EXTERNAL

Public Information Notice

RELATIONS

DEPARTMENT

Public Information Notice (PIN) No. 10/152

FOR IMMEDIATE RELEASE

December 2, 2010

International Monetary Fund

$70019^{\text {th }}$ Street, NW

Washington, D. C. 20431 USA

\section{IMF Executive Board Concludes 2010 Article IV Consultation with Cape Verde}

On November 22, 2010, the Executive Board of the International Monetary Fund (IMF) concluded the Article IV consultation with Cape Verde. ${ }^{1}$

\section{Background}

Cape Verde sustained strong economic growth during 2006-08, reduced the poverty rate and graduated from the UN least-developed country (LDC) status. During this period, the government built up fiscal buffers, which it used for fiscal stimulus in 2009 to limit the impact of weaker external demand from the global crisis.

The authorities' economic program of 2006-10 was supported by an arrangement with the IMF under the Policy Support Instrument (PSI). Policy implementation was strong, with some assessment criteria met ahead of schedule. Following the expiration of this program in July 2010, the government has requested that its economic policies for 2011 be supported by a new 15-month PSI program. The government's medium-term development strategy is set out in the Poverty Reduction Strategy Paper (PRSP), which focuses on further reducing poverty and promoting private sector-led growth.

Leading economic indicators point to a solid rebound in activity, with GDP growth projected at about 51/2 percent in 2010. Inflation has edged up to 3 percent in the 12 months to August 2010, mainly because of higher prices in service activities and food. In the first half of 2010, international trade grew moderately and foreign direct investment stabilized. The outlook for service exports in the second half of 2010 is favorable, as tourist establishments report high

\footnotetext{
${ }^{1}$ Under Article IV of the IMF's Articles of Agreement, the IMF holds bilateral discussions with members, usually every year. A staff team visits the country, collects economic and financial information, and discusses with officials the country's economic developments and policies. On return to headquarters, the staff prepares a report, which forms the basis for discussion by the Executive Board. At the conclusion of the discussion, the Managing Director, as Chairman of the Board, summarizes the views of Executive Directors, and this summary is transmitted to the country's authorities. An explanation of any qualifiers used in summings up can be found here: http://www.imf.org/external/np/sec/misc/qualifiers.htm.
} 
2

reservation rates for the high season. Over the medium term, growth rates of exports and Foreign Direct Investment (FDI) are expected to pick up as global conditions improve.

Fiscal policy aims at improving infrastructure while maintaining fiscal sustainability. Fiscal deficits of 6.3 percent of GDP and 13.5 percent of GDP in 2009 and 2010, respectively, reflect the temporary acceleration of the public investment program as counter-cyclical stimulus. The pace of public investment spending is projected to slow over the next couple of years in order to ensure debt sustainability. Fiscal policy continues to support the peg, and domestic debt is kept below 20 percent of GDP.

The Bank of Cape Verde (BCV) raised its policy rate in late 2008 against the backdrop of higher global risk premia associated with the international crisis. Following a decline in global risk indicators, the BCV reduced its policy rate by one percentage point, to 4.25 percent, in January 2010, keeping the spread with the Euribor wide. To support the peg, monetary policy focuses on ensuring gradual reserve accumulation by encouraging inflows of emigrant deposits and restraining private sector credit that would fuel imports.

On the structural front, the government's reforms focus on reducing the fiscal risk arising from state owned enterprises. The authorities have put the public electricity company, Electra, and the airline company, TACV, on a restructuring plan to address their structural losses.

\section{Executive Board Assessment}

Executive Directors commended the Cape Verde authorities on the recent strong macroeconomic performance and the substantial progress toward achieving the MDGs. Directors welcomed the economy's resilience to recent adverse shocks and the signs of a solid recovery in 2010. Prudent macroeconomic management permitted the implementation of effective counter-cyclical policies and supports the implementation of the government's medium-term development strategy. The new PSI-supported program will help the authorities build on their strong performance by maintaining macroeconomic discipline through the upcoming election period and further reducing vulnerabilities to economic conditions in trading partners.

Directors supported the authorities' fiscal stance as being appropriately balanced between the implementation of the ambitious public investment program and the need to safeguard debt sustainability. They stressed the importance of making judicious use of available concessional funds and selecting foreign-financed projects on the basis of pro-growth or pro-poor objectives. Directors welcomed the authorities' intention to unwind the expansionary fiscal stance starting from 2011 and to scale back foreign borrowing in order to rebuild fiscal policy buffers, while developing a medium-term debt strategy by June 2011. They called for continued reform efforts to strengthen public revenues and to improve the performance of loss-making state owned enterprises.

Directors noted the staff assessment that the escudo remains broadly aligned with its fundamentals while recognizing that the analysis is subject to a high degree of uncertainty. They stressed the importance of prudent fiscal management to support the exchange rate peg and continued structural reforms to foster productivity growth in the private sector to boost competitiveness. 
Directors agreed that the monetary stance is appropriate, and noted that, given the fragile global environment, shoring up reserve accumulation by stabilizing remittance inflows is justified. Directors welcomed the authorities' efforts to improve liquidity management and monetary operations, which should enhance policy effectiveness. They noted that an improved monetary transmission mechanism would allow the authorities to better influence market interest rates, which should be brought in line with the Euribor over time to mitigate the risks of speculative inflows.

Directors were encouraged by the recent measures taken by the BCV to safeguard financial stability. They welcomed the new draft banking law and the improvements to the regulatory and supervisory framework. Directors looked forward to further measures to boost supervisory capacity and to continue progress with the implementation of Financial Sector Assessment Program recommendations.

Public Information Notices (PINs) form part of the IMF's efforts to promote transparency of the IMF's views and analysis of economic developments and policies. With the consent of the country (or countries) concerned, PINs are issued after Executive Board discussions of Article IV consultations with member countries, of its surveillance of developments at the regional level, of post-program monitoring, and of ex post assessments of member countries with longer-term program engagements. PINs are also issued after Executive Board discussions of general policy matters, unless otherwise decided by the Executive Board in a particular case. 
Cape Verde: Selected Economic and Financial Indicators, 2007-11

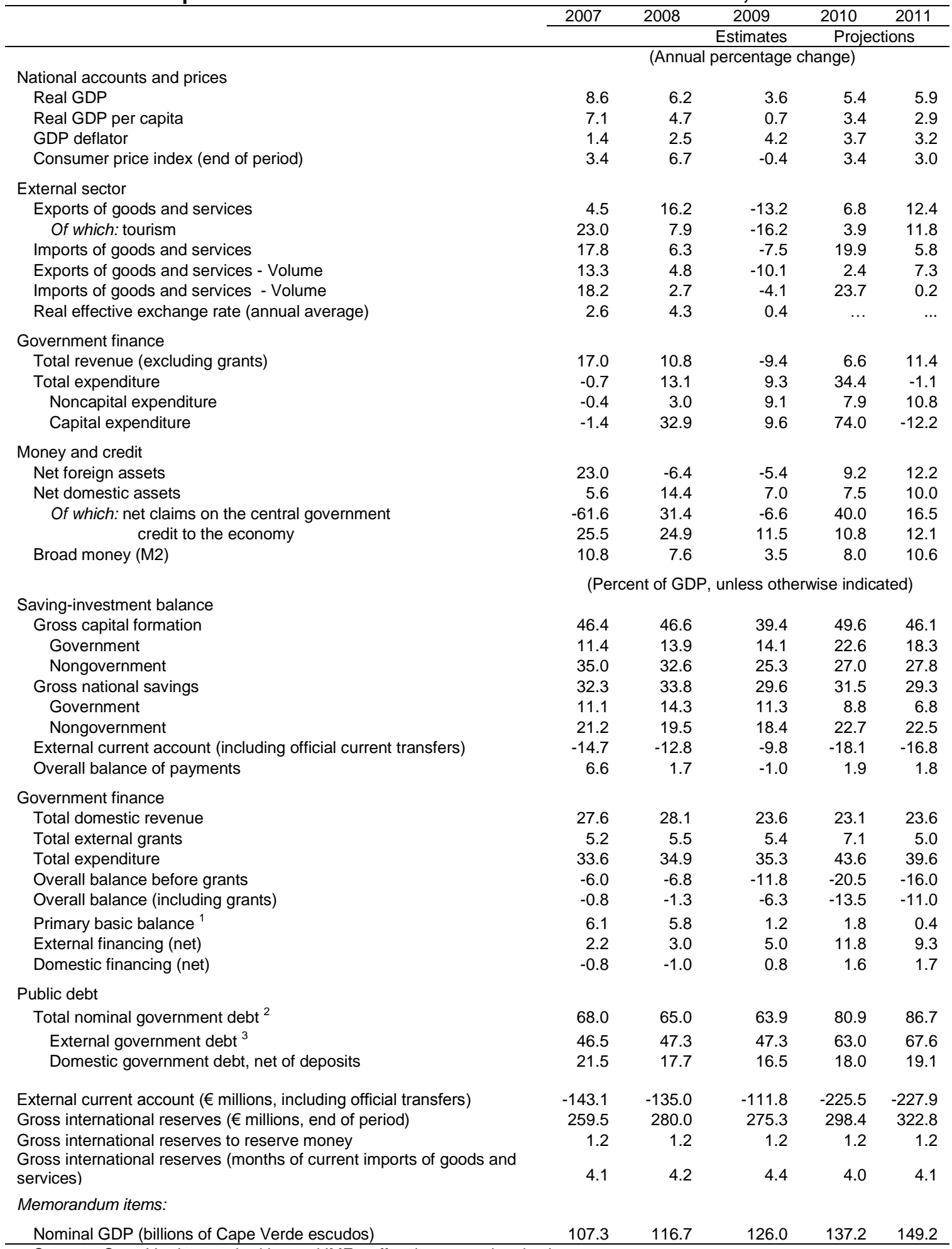

Sources: Cape Verdean authorities and IMF staff estimates and projections.

${ }^{1}$ Excluding grants, interest payments, and foreign financed

${ }^{2}$ Net of central government deposits; including verified stock of domestic and external arrears.

${ }^{3}$ Excluding claims on the offshore Trust Fund. 
Press Release No. 10/457

International Monetary Fund

FOR IMMEDIATE RELEASE

Washington, D.C. 20431 USA

November 23, 2010

\section{IMF Executive Board Approves a 15-Month Policy Support Instrument for Cape Verde}

The Executive Board of the International Monetary Fund (IMF) approved on November 22, 2010 a 15-month Policy Support Instrument (PSI) for Cape Verde. The IMF's framework for PSIs is designed for low-income countries that may not need IMF financial assistance, but still seek IMF advice, monitoring, and endorsement of their policy frameworks. PSIsupported programs are based on country-owned poverty reduction strategies adopted in a participatory process involving civil society and development partners. (See Public Information Notice No. 05/145).

The PSI for Cape Verde aims to consolidate macroeconomic stability, and achieve sustained broad-based growth. The authorities' program will build on the macroeconomic success and structural reforms of the previous PSI supported program, and will help maintain macroeconomic discipline.

Following the Executive Board's discussion of Cape Verde, Mr. Murilo Portugal, Deputy Managing Director and Acting Chair, stated:

"Cape Verde is rebounding from the global economic crisis, driven by a pickup in tourism and construction. Inflation is low and the real value of the escudo remains competitive. The authorities' strong record of policy implementation over the past several years succeeded in building fiscal and reserves buffers, which provided room for counter-cyclical macroeconomic policies to moderate the impact of the global slowdown.

"The policies supported by a new 15-month Policy Support Instrument will pursue national development objectives for increasing growth, diversifying the economy and making progress on social policies. Given these objectives, the authorities have implemented a temporary acceleration of the public investment program, which is mainly financed with concessional external resources. In order to rebuild fiscal buffers against future adverse external shocks, the authorities have committed to scale back foreign-financed spending from next year and to enhance debt management capacity. In response to weaker-than-expected 
revenue, they have also restrained non-priority spending while protecting social spending on vulnerable groups. These policies are welcome, as prudent fiscal management is crucial in supporting the exchange rate peg.

"Given the fragile global environment, the Bank of Cape Verde is appropriately focused on shoring up reserve accumulation by stabilizing inflows of emigrant deposits. Improving the monetary transmission mechanism will allow the authorities to better influence market interest rates, which should be brought in line with the Euribor over time to mitigate the risks of speculative inflows. The authorities' intention of bringing all banks under a single banking law is welcome. Continued efforts to enhance banking supervision and to develop the government securities market, in line with FSAP recommendations, will help safeguard financial system soundness and enhance the transmission of monetary policy”, Mr. Portugal added.

ANNEX

\section{Recent Economic Developments}

Cape Verde weathered the crisis well. Growth slowed in 2009, but remained strong compared to global trends. The global slowdown had the largest impact on tourism, FDI flows, and private construction. The authorities used the policy space created by prudent fiscal policies in recent years and additional inexpensive external financing to accelerate the public investment program that was already in place. Inflation declined from 6.8 percent (annual average) in 2008 to 1 percent in 2009 and should remain around 2 percent in the medium term. There are signs of recovery in 2010; and the growth rate in 2010 should pick up as the public investment program and the global recovery favorably affects the construction, tourism, and transportation sectors. Growth should then gradually rise in the medium term.

The challenge for Cape Verde is to take advantage of the current window of highly concessional financing while ensuring debt sustainability. The pace of the investment program is expected to continue in 2011-12, but should return to more moderate levels after the completion of current large infrastructure projects. The investment program is intended to support long-term private growth based on services exports in line with the Cape Verde Poverty Reduction and Growth Strategy.

\section{Program Summary}

Cape Verde's PSI will continue to build foreign reserves and keep the domestic debt stock low to support the exchange rate peg. In order to protect international reserves, net domestic borrowing will be contained. Fiscal policy will be orientated toward gradual exit from the current counter-cyclical fiscal measures. In 2010-11, fiscal policy will remain anchored on 
implementing the medium term public investment program and minimizing and prudently managing debt. As the pace of public investment slows, fiscal deficit is expected to decrease from 13.5 percent of GDP in 2010 to 7.7 percent in 2012. External borrowing will continue, mostly on concessional terms, and will be scaled back after the completion of the current large infrastructure projects. The government will continue to address contingent liabilities arising from state owned enterprises (SOEs). In accordance with its poverty reduction strategy, the government is committed to safeguarding priority spending. In this regard, the authorities will implement active policies aimed at supporting sustainable solutions to unemployment and at fostering the integration of the most vulnerable into the economic and social life of the country. The government will invest heavily in the provision of training that caters to current labor market needs, particularly among the youth. Skills development are also supported by investments on housing, health, water and sanitation, all of which conducive to a better and more productive life for Cape Verdeans. 
Cape Verde: Selected Economic and Financial Indicators, 2007-11



Sources: Cape Verdean authorities, and IMF staff estimates and projections.

${ }^{1}$ Excluding grants, interest payments, and foreign financed investments

${ }^{2} \mathrm{Net}$ of central government deposits; including verified stock of domestic and external arrears.

${ }^{3}$ Excluding claims on the offshore Trust Fund. 


\section{Statement by Lauren W. Rutayisire, Executive Director for Cape Verde November 22, 2010}

The 2006-10 Policy Support Instrument (PSI) served Cape Verde well, notably by playing a valuable catalytic role in support of the authorities' policy and reform agenda and efforts to improve the country's market access. As noted in the staff report, performance was strong under the PSI. This paved the way for increased macroeconomic and financial stability, sustained economic growth, and significant poverty reduction. While these positive achievements reflect primarily the authorities' strong commitment to good economic governance, they were also facilitated by the valuable policy advice and technical assistance provided by the Fund.

In order to consolidate these achievements, the authorities request a 15-month PSI. The proposed PSI-supported program is geared toward sustaining macroeconomic stability, strengthening public finance and debt management, providing adequate support for the exchange rate peg, and ensuring continued financial sector stability and development. To achieve these objectives, the authorities will continue to make inroads in containing domestic debt and strengthening debt management, sustaining comfortable level of foreign reserves, and implementing prudent macroeconomic policies. Cape Verde's strong performance under the last PSI arrangement augurs well for the implementation of the proposed program supported by the PSI. At the same time, the country's macroeconomic fundamentals meet the PSI requirements, as noted in the staff report. In particular, the country's fiscal deficit is expected to decline steadily going forward after having increased significantly in recent years, as the authorities seized a time-bound opportunity to finance highly profitable infrastructure projects with concessional resources.

\section{Policy and Reform Agenda for 2010 Onwards}

Sound macroeconomic policies played a key role in mitigating the impact of the global crisis. Amid the recent global crisis, the authorities' prudent economic management paid off, providing space for much-needed countercyclical policies and pro-poor spending. Going forward, the authorities will persevere in the implementation of the policy and reform agenda set forth to achieve the objectives of PSI-supported policies.

\section{Fiscal Policy and Reforms}

The authorities are committed to the continuation of prudent fiscal management, notably through strict control over expenditures. Going forward, the fiscal stance is expected to improve significantly as the rapid implementation of the ongoing public investment program recedes. In this regard, the overall fiscal deficit is forecast to decline by about 5 points of GDP within the next two years. At the same time, care will continue to be taken to protect priority spending, particularly those in favor of the poorest segments of the population. In 
this connection, a broad range of pro-poor initiatives are being implemented, including social security reform, training programs, and investments in social sectors.

Significant strides will be made in advancing the fiscal reform agenda. In 2011, the authorities will continue to adhere to the established practice of conducting a mid-year fiscal review. As part of the fiscal reform agenda, the draft individual and corporate income tax bills are scheduled to be submitted to the National Assembly by the end of the first half of 2011.

The authorities' reform agenda also encompasses the reform of state-owned enterprises with a view to containing fiscal risks emanating from their operations. Key among the steps taken to facilitate the successful restructuring of public enterprises was the recent enactment of the Law of State Enterprises, Public Managers, and Corporate Good Governance which improved the regulatory framework for managing SOEs. Furthermore, the restructuring plan for the electricity company Electra that is underway is expected to help contain operating costs and promote private sector involvement. The airline company TACV registered last year a marked improvement in its income situation and proceeded recently to the clearance of its arrears to private providers. For the purpose of ensuring increased fiscal transparency and accountability, a report will be prepared that summarizes contingent liabilities emanating from SOEs as well as cross debt that exists between them.

\section{Debt Management}

Going forward, debt management will be geared toward reducing the debt-to-GDP ratio. Domestic debt is expected to remain below 20 percent of GDP, thus providing support for the exchange rate peg by helping maintain international reserves at comfortable levels. The authorities intend to develop a medium-term debt strategy (MTDS) that will be presented to the Council of Ministers in June 2011. The MTDS is expected to be instrumental in helping manage public debt, especially since borrowing on nonconcessional terms is anticipated to increase amid the increasingly limited availability of concessional resources that is expected to result from Cape Verde's graduation from least-developed-country status.

As previously noted, the authorities are thus taking advantage of the time-bound availability of concessional resources to implement ongoing large infrastructure projects. While pressures on debt ratios are anticipated as a result of the implementation of these projects, the high profitability of these projects which is illustrated by studies with the assistance of the country's partners is expected to further strengthen debt sustainability in the medium term. It is also noteworthy that upon completion of the ongoing PIP, capital spending financed by external loans will be expected to decrease sharply. 


\section{Monetary Policy and Financial Sector Reform}

Monetary policy will continue to be conducted by the Bank of Cape Verde (BCV) with a view to supporting the exchange rate system and stabilizing the economy in the context of a largely open capital account. Its conduct will benefit from the authorities' stated commitment to improve the coordination of fiscal and monetary policy. In this regard, fiscal and monetary authorities plan to sign by the end of this year an agreement by which the BCV will make treasury securities the key instrument for monetary operations. It is also the authorities' intention to take necessary measures to develop the government security market notably through the issuance of treasury bonds and recourse to auctions and information technology to facilitate securities transactions.

Preserving financial stability will continue to rank high on the authorities' agenda. In this respect, the BCV will work toward further strengthening its regulatory and supervisory framework. From 2011 onwards, it plans on issuing regularly a financial stability report. At the same time, it will keep monitoring closely banks' soundness indicators in line with established practice. Going forward, financial stability will also be served by the planned harmonization of prudential norms between onshore and offshore banking activities. The draft legislation that will be prepared to this effect is scheduled to be submitted to the parliament by the end of June 2011. In the meantime, the Finance Minister and the BCV Governor intend to co-sign a MOU that will allow the finance ministry staff to integrate the BCV standing financial stability committee. It is expected that this MOU will define formally crisis management and information-sharing mechanisms to be put in place by the two institutions whenever appropriate to address a crisis situation in an effective and collaborative manner.

In light of Cape Verde's continued strong program and economic performance, we call on Directors to consider favorably the authorities' request for a new PSI arrangement. 